\title{
Teorema Fundamental das Imersões e Superfícies Mínimas em Espaços Produto
}

Fernando Maia Nardelli Escobosa

\author{
DisSERTAÇÃO APRESENTADA \\ $\mathrm{AO}$ \\ Instituto De Matemática e EstatísticA \\ DA \\ Universidade DE SÃo PAUlo \\ PARA \\ OBTENÇÃO DO TÍTULO \\ DE \\ Mestre EM CiÊnCIAS \\ Programa: Matemática \\ Orientador: Prof. Dr. Alexandre Lymberopoulos
}

Durante o desenvolvimento deste trabalho o autor recebeu auxílio financeiro da $\mathrm{CNPq}$

São Paulo, fevereiro de 2017 


\section{Teorema Fundamental das Imersões e Superfícies Mínimas em Espaços Produto}

Esta versão da dissertação contém as correções e alterações sugeridas pela Comissão Julgadora durante a defesa da versão original do trabalho, realizada em 22/02/2017. Uma cópia da versão original está disponível no

Instituto de Matemática e Estatística da Universidade de São Paulo.

Comissão Julgadora:

- Prof. Dr. Alexandre Lymberopoulos (orientador) - IME-USP

- Prof. Dr. Fernando Manfio - ICMC-USP

- Prof. Dr. Márcio Fabiano da Silva - UFABC 


\section{Agradecimentos}

Agradecer de forma segura a todas as pessoas que, direta ou indiretamente, fizeram parte da minha trajetória ao longo deste mestrado não é uma tarefa fácil. Quero demonstrar meus sentimentos àqueles que me auxiliaram das mais diversas formas a cumprir este objetivo e dar mais um passo na minha carreira acadêmica.

Primeiramente à Deus, responsável pela força diária e motivação para nunca desistir;

À minha família, por me apoiar, ser meu ponto de equilíbrio e me dar confiança nas minhas capacidades;

À minha namorada, por estar sempre presente, me trazendo conforto e alegria todos os dias;

Ao meu orientador, pelo conhecimento, incentivo e longas horas de discussão, sempre acreditando no meu potencial;

À professora Rosa Maria S. B. Chaves, pela constante disponibilidade e estímulo que vão além deste trabalho;

Aos colegas e amigos do IME-USP, da graduação, mestrado e doutorado, pelas conversas e ajudas nos bons (e não tão bons) momentos;

Aos professores do IME-USP que prestaram sua contribuição para minha formação e avanço dentro da investigação científica;

À CNPq, cujo fomento permitiu a conclusão desta dissertação.

Maturidade e perseverança são virtudes alcançadas com tempo e esforço, que só foram possíveis graças a vocês. A todos, meus sinceros agradecimentos. 


\section{Resumo}

Escobosa, F. M. N. Teorema Fundamental das Imersões e Superfícies Mínimas em Espaços Produto. 2017. 86 f. Dissertação de Mestrado - Instituto de Matemática e Estatística, Universidade de São Paulo, São Paulo, 2017.

Neste trabalho demonstramos o Teorema Fundamental das Imersões para $\mathbb{S}^{m} \times \mathbb{R}$ e $\mathbb{H}^{m} \times \mathbb{R}$, dando condições necessárias e suficientes para que uma variedade Riemanniana simplesmente conexa seja isometricamente imersa nestes ambientes. Para isto, utilizamos referenciais móveis e distribuições integráveis. Como aplicação do Teorema Fundamental, provamos a existência de uma família a um parâmetro de deformações isométricas mínimas de uma dada superfície mínima em $\mathbb{S}^{2} \times \mathbb{R}$ e $\mathbb{H}^{2} \times \mathbb{R}$, chamada de família associada. Além disso, relacionamos o problema de encontrar uma imersão isométrica mínima para uma dada superfície Riemanniana simplesmente conexa nestes espaços a um sistema de duas equações diferenciais parciais. Construímos exemplos de superfícies conjugadas em ambos os ambientes e de superfícies admitindo duas imersões mínimas isométricas não associadas em $\mathbb{H}^{2} \times \mathbb{R}$.

Palavras-chave: imersões isométricas, superfícies mínimas, família associada. 


\section{Abstract}

Escobosa, F. M. N. Fundamental Theorem of Immersions and Minimal Surfaces in Product Spaces. 2017. 86 f. Dissertação de Mestrado - Instituto de Matemática e Estatística, Universidade de São Paulo, São Paulo, 2017.

In this work we give a proof of the Fundamental Theorem of Immersions for $\mathbb{S}^{m} \times \mathbb{R}$ and $\mathbb{H}^{m} \times \mathbb{R}$, providing necessary and sufficient conditions for a simply connected Riemannian manifold to be isometrically immersed on this ambient spaces. In order to do this, we use moving frames and integrable distributions. As an application of the Fundamental Theorem, we proof the existence of a one parameter family of minimal isometric deformations of a given minimal surface in $\mathbb{S}^{2} \times \mathbb{R}$ and $\mathbb{H}^{2} \times \mathbb{R}$, which is called the associated family. Furthermore, we relate the problem of finding an minimal isometric immersion for a given simply connected Riemannian surface in this spaces to a system of two partial differential equations. Also, we construct examples of conjugated surfaces in both ambient spaces and surfaces admitting two non associated minimal isometric immersions in $\mathbb{H}^{2} \times \mathbb{R}$.

Keywords: isometric immersions, minimal surfaces, associated family. 


\section{Sumário}

$\begin{array}{ll}\text { Introdução } & 1\end{array}$

1 Teoria de Subvariedades $\quad 3$

1.1 Subvariedades Riemannianas . . . . . . . . . . . . . . . . . . 3

1.2 O Método do Referencial Móvel . . . . . . . . . . . . . . . . . . . . . 4

1.3 Subvariedades de $\mathbb{S}^{m} \times \mathbb{R}$ e $\mathbb{H}^{m} \times \mathbb{R} \ldots \ldots \ldots \ldots$

2 Imersões Isométricas $\quad 17$

2.1 As Equações de Compatibilidade . . . . . . . . . . . . . . . . . . . . . . . . . . . . . . . .

2.2 Imersões isométricas em $\mathbb{S}^{m} \times \mathbb{R}$ e $\mathbb{H}^{m} \times \mathbb{R} \ldots \ldots \ldots \ldots \ldots \ldots \ldots$

3 Imersões Mínimas $\quad 39$

3.1 A Família Associada . . . . . . . . . . . . . . . . . . . . . . . 40

3.2 Superfícies Parametrizadas: Como Fazer as Contas e Propriedades . . . . . . . . . . 51

3.3 Superfícies Conjugadas em $\mathbb{S}^{2} \times \mathbb{R} \ldots \ldots \ldots \ldots \ldots \ldots$

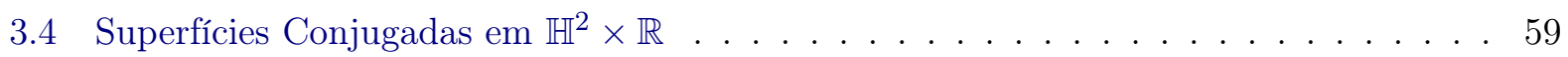

3.5 Mais exemplos no caso hiperbólico . . . . . . . . . . . . . . . . 73

$\begin{array}{ll}\text { Referências Bibliográficas } & 75\end{array}$ 


\section{Introdução}

Em 1867, Bonnet mostrou em [Bon67] que dadas duas formas quadráticas definidas em um aberto $U \subset \mathbb{R}^{2}$ que satisfazem as equações de Gauss e Codazzi, fundamentais para o estudo de subvariedades Riemannianas, então existe uma única imersão $f: U \rightarrow \mathbb{R}^{3}$, cuja métrica induzida e a segunda forma fundamental são as formas quadráticas dadas. É sabido que, para formas espaciais de dimensão $n$, as equações de Gauss e Codazzi estão intrinsecamente definidas em suas subvariedades e são condições suficientes para que uma variedade Riemanniana de dimensão $n-1$ simplesmente conexa seja isometricamente imersa nestes espaços. Uma demonstração deste fato pode ser encontrado em [Spi79].

Em geral, as equações de Gauss e Codazzi não são intrinsecamente definidas em subvariedades. Além disso, para casos em que a codimensão é maior do que um, necessitamos trabalhar também com uma outra equação, a equação de Ricci. Neste trabalho, baseado principalmente nas referências [Dan09] e [CC10], determinamos condições necessárias e suficientes para que uma variedade Riemanniana $M$ de dimensão $n$ simplesmente conexa possa ser isometricamente imersa em $\mathbb{S}^{m} \times \mathbb{R}$ ou $\mathbb{H}^{m} \times \mathbb{R}$, onde $n \leq m$. Este resultado será chamado de Teorema Fundamental das Imersões, e sua demonstração é baseada no metódo utilizado por Tenenblat em [Ten71], que demonstrou este Teorema para o caso Euclidiano.

Um outro fato bem conhecido em $\mathbb{R}^{3}$ é a existência de uma família a um parâmetro de deformações de imersões isométricas mínimas de uma dada superfície mínima, chamada de família associada, obtida rotacionando o operador forma. Por exemplo, o catenoide e o helicoide pertencem à mesma família. Além disso, duas imersões isométricas mínimas de uma mesma superfície Riemanniana em $\mathbb{R}^{3}$ pertencem à mesma família, como pode ser visto em [Spi79]. Como aplicação do Teorema Fundamental mencionado acima, provamos a existência de uma família associada de imersões isométricas mínimas em $\mathbb{S}^{2} \times \mathbb{R}$ ou $\mathbb{H}^{2} \times \mathbb{R}$, análoga ao caso Euclidiano. A existência da família associada nestes ambientes foi independentemente provada também por Hauswirth, Sa Earp e Toubina em [HSET08], utilizando outras técnicas. Ainda como aplicação do Teorema Fundamental, seguindo [Dan15], relacionamos o problema de encontrar uma imersão isométrica mínima para uma dada superfície Riemanniana simplesmente conexa nestes espaços a um sistema de duas equações diferenciais parciais. Em seguida, mostramos que, em $\mathbb{S}^{2} \times \mathbb{R}$, helicoides e onduloides são conjugadas, e em $\mathbb{H}^{2} \times \mathbb{R}$, helicoides são conjugados a catenoides ou a superfícies mínimas folheadas por curvas horizontais de curvatura constante. 


\section{Capítulo 1}

\section{Teoria de Subvariedades}

Começaremos este capítulo recordando brevemente algumas definições e resultados básicos de geometria Riemanniana e da teoria de subvariedades Riemannianas. As referências que utilizamos para tais conceitos foram [dC79] e [Daj90].

Após feito isso, introduziremos o método do referencial móvel para variedades e subvariedades Riemannianas, definindo as chamadas formas de conexão e demonstrando suas principais relações que serão utilizadas no decorrer deste trabalho, principalmente no capítulo 2. Para mais detalhes sobre o método do referencial móvel, indicamos [dC76].

Terminaremos este capítulo estudando a geometria das subvariedades dos espaços produto $\mathbb{S}^{n} \times \mathbb{R}$ e $\mathbb{H}^{n} \times \mathbb{R}$, e aplicaremos tudo o que fizemos nas duas primeiras seções para este caso específico.

\subsection{Subvariedades Riemannianas}

Seja $\bar{M}$ uma variedade Riemanniana de dimensão $m$ com métrica $g$. Seja $M \subset \bar{M}$ uma subvariedade Riemanniana mergulhada de dimensão $n<m$, ou seja, a inclusão $i: M \rightarrow \bar{M}$ é um mergulho e a métrica em $M$ é dada por $i^{*} g$. Como a métrica $i^{*} g$ é apenas a restrição de $g$ a $T M$, vamos denotar por $g$ ambas as métricas de $\bar{M}$ e $M$.

Para cada ponto $x \in M$, podemos considerar $T_{x} M$ como subespaço vetorial de $T_{x} \bar{M}$, logo a métrica $g$ induz a decomposição de $T_{x} \bar{M}$ na soma direta

$$
T_{x} \bar{M}=T_{x} M \oplus T_{x} M^{\perp}
$$

onde $T_{x} M^{\perp}$ denota o complemento ortogonal de $T_{x} M$ em $T_{x} \bar{M}$. Desta maneira obtemos o fibrado vetorial $T M^{\perp}=\bigcup_{x \in M} T_{x} M^{\perp}$, chamado de fibrado normal de $M$. Projetando ortogonalmente em cada ponto $x \in M$ em $T_{x} M$ e $T_{x} M^{\perp}$, definimos em $\left.T \bar{M}\right|_{M}=\bigcup_{x \in M} T_{x} \bar{M}$ as projeções tangencial e normal:

$$
\begin{aligned}
& ()^{\top}:\left.T \bar{M}\right|_{M} \rightarrow T M \\
& ()^{\perp}:\left.T \bar{M}\right|_{M} \rightarrow T M^{\perp},
\end{aligned}
$$

de maneira que para cada $\bar{X} \in \Gamma\left(\left.T \bar{M}\right|_{M}\right),(\bar{X})^{\top} \in \Gamma(T M)$ é a componente tangencial de $\bar{X}$ e $(\bar{X})^{\perp} \in \Gamma\left(T M^{\perp}\right)$ é a componente normal de $\bar{X}$.

Denote por $\nabla$ e $\bar{\nabla}$ as conexões Riemannianas e por $R$ e $\bar{R}$ os tensores curvatura de $M$ e $\bar{M}$, respectivamente. Usaremos a convenção $R(X, Y)=\nabla_{Y} \nabla_{X}-\nabla_{X} \nabla_{Y}+\nabla_{[X, Y]}$, para $X, Y \in \Gamma(T M)$. Além disso, denotaremos por $\nabla^{\perp}$ a conexão normal e por $R^{\perp}$ o tensor curvatura normal de $M$.

Definimos a segunda forma fundamental $I I: \Gamma(T M) \times \Gamma(T M) \rightarrow \Gamma\left(T M^{\perp}\right)$ de M por

$$
I I(X, Y)=\left(\bar{\nabla}_{X} Y\right)^{\perp} .
$$


Como $\nabla=(\bar{\nabla})^{\top}$, obtemos a Fórmula de Gauss:

$$
\bar{\nabla}_{X} Y=\nabla_{X} Y+I I(X, Y) .
$$

Note que $I I$ é simétrica e bilinear sobre $C^{\infty}(M)$.

Para $X, Y \in \Gamma(T M)$ e $\mu \in \Gamma\left(T M^{\perp}\right)$, o operador forma (ou operador de Weingarten) $A_{\mu}$ é definido por $g\left(A_{\mu}(X), Y\right)=g(I I(X, Y), \mu)$. Então a aplicação $A_{\mu}: \Gamma(T M) \rightarrow \Gamma(T M)$ é linear sobre $C^{\infty}(M)$ e $g\left(A_{\mu}(X), Y\right)=g\left(X, A_{\mu}(Y)\right)$, ou seja, $A_{\mu}$ é auto-adjunto em relação a $g$. Utilizando a Fórmula de Gauss é possível mostrar que $A_{\mu}(X)=-\left(\bar{\nabla}_{X} \mu\right)^{\top}$, e obtemos a Fórmula de Weingarten:

$$
\bar{\nabla}_{X} \mu=-A_{\mu}(X)+\nabla_{X}^{\perp} \mu \text {. }
$$

Utilizando as Fórmulas de Gauss e Weingarten é possível mostrar que para $X, Y, Z, W \in \Gamma(T M)$ e $\eta, \xi \in \Gamma\left(T M^{\perp}\right)$ valem as seguintes equações:

i) Equação de Gauss:

$$
\begin{aligned}
g(R(X, Y) Z, W)= & g(\bar{R}(X, Y) Z, W)+g(I I(X, Z), I I(Y, W)) \\
& -g(I I(X, W), I I(Y, Z))
\end{aligned}
$$

ii) Equação de Codazzi:

$$
\begin{aligned}
(\bar{R}(X, Y) \eta)^{\top}= & \left(\nabla_{X} A_{\eta}\right)(Y)-\left(\nabla_{Y} A_{\eta}\right)(X) \\
& +A_{\nabla_{\bar{Y}}} X-A_{\nabla_{X} \frac{1}{X} \eta} Y
\end{aligned}
$$

iii) Equação de Ricci:

$$
g(\bar{R}(X, Y) \eta, \xi)=g\left(R^{\perp}(X, Y) \eta, \xi\right)+g\left(\left[A_{\eta}, A_{\xi}\right](X), Y\right) .
$$

onde $\left(\nabla_{X} A_{\alpha}\right)(Y)=\nabla_{X} A_{\alpha}(Y)-A_{\alpha}\left(\nabla_{X} Y\right)$.

Uma demonstração destas equações pode ser encontrada em [dC79].

\subsection{O Método do Referencial Móvel}

Seja $\bar{M}$ uma variedade Riemanniana de dimensão $m$, com métrica $\langle$,$\rangle , conexão Riemanniana$ $\bar{\nabla}$ e tensor curvatura $\bar{R}$. Sejam $\left\{e_{A}\right\}_{A=1}^{m}$ um referencial ortonormal local em $\bar{M}$ e $\left\{\omega^{A}\right\}_{A=1}^{m}$ a base dual de $\left\{e_{A}\right\}_{A=1}^{m}$, ou seja,

$$
\omega^{A}\left(e_{B}\right)=\delta_{A B} .
$$

Definimos as 1-formas $\left\{\omega_{B}^{A}\right\}_{A, B=1}^{m}$ em $\bar{M}$ por

$$
\omega_{B}^{A}(X)=\left\langle\bar{\nabla}_{X} e_{B}, e_{A}\right\rangle,
$$

para todo $X \in \Gamma(T \bar{M})$.

Definição 1.2.1. As formas $\omega_{B}^{A}$ dadas por (1.4) são chamadas as formas de conexão de $\bar{M}$ no referencial $\left\{e_{A}\right\}$.

Note que como $\left\langle e_{B}, e_{A}\right\rangle=\delta_{B A}$, então $\left\langle\bar{\nabla}_{X} e_{B}, e_{A}\right\rangle=-\left\langle e_{B}, \bar{\nabla}_{X} e_{A}\right\rangle$, ou seja, $\omega_{A}^{B}=-\omega_{B}^{A}$. Além disso temos que

$$
\bar{\nabla}_{X} e_{B}=\sum_{A} \omega_{B}^{A}(X) e_{A}
$$


e podemos escrever $\omega_{B}^{A}$ na base $\left\{\omega^{A}\right\}$ :

$$
\omega_{B}^{A}=\sum_{C}\left\langle\bar{\nabla}_{e_{C}} e_{B}, e_{A}\right\rangle \omega^{C}
$$

Definimos também $\bar{R}_{B C D}^{A}=\left\langle R\left(e_{C}, e_{D}\right) e_{B}, e_{A}\right\rangle$.

A próxima Proposição relaciona as derivadas exteriores da base $\left\{\omega^{A}\right\}$ com as formas de conexão.

Proposição 1.2.2. Com as definições dadas acima, temos que

$$
d \omega^{A}+\sum_{B} \omega_{B}^{A} \wedge \omega^{B}=0
$$

Demonstração. Lembrando que (Veja [Lee12]) dada uma 1-forma $\omega$ em $\bar{M}$, se $X, Y \in \Gamma(T \bar{M})$ então

$$
d \omega(X, Y)=X \omega(Y)-Y \omega(X)-\omega([X, Y])
$$

temos

$$
\begin{aligned}
d \omega^{A}\left(e_{C}, e_{D}\right) & =e_{C} \omega^{A}\left(e_{D}\right)-e_{D} \omega^{A}\left(e_{C}\right)-\omega^{A}\left(\left[e_{C}, e_{D}\right]\right) \\
& =e_{C}\left(\delta_{A D}\right)-e_{D}\left(\delta_{A C}\right)-\omega^{A}\left(\bar{\nabla}_{e_{C}} e_{D}-\bar{\nabla}_{e_{D}} e_{C}\right) \\
& =-\omega^{A}\left(\bar{\nabla}_{e_{C}} e_{D}\right)+\omega^{A}\left(\bar{\nabla}_{e_{D}} e_{C}\right) \\
& =-\omega^{A}\left(\sum_{B} \omega_{D}^{B}\left(e_{C}\right) e_{B}\right)+\omega^{A}\left(\sum_{B} \omega_{C}^{B}\left(e_{D}\right) e_{B}\right) \\
& =-\omega_{D}^{A}\left(e_{C}\right)+\omega_{C}^{A}\left(e_{D}\right) .
\end{aligned}
$$

Por outro lado,

$$
\begin{aligned}
\sum_{B} \omega_{B}^{A} \wedge \omega^{B}\left(e_{C}, e_{D}\right) & =\sum_{B}\left[\omega_{B}^{A}\left(e_{C}\right) \omega^{B}\left(e_{D}\right)-\omega_{B}^{A}\left(e_{D}\right) \omega^{B}\left(e_{C}\right)\right] \\
& =\omega_{D}^{A}\left(e_{C}\right)-\omega_{C}^{A}\left(e_{D}\right)
\end{aligned}
$$

Logo,

$$
d \omega^{A}\left(e_{C}, e_{D}\right)+\sum_{B} \omega_{B}^{A} \wedge \omega^{B}\left(e_{C}, e_{D}\right)=0
$$

Suponha agora que $M \subset \bar{M}$ é uma subvariedade riemanniana de $\bar{M}$ de dimensão $n<m$ com métrica $\langle$,$\rangle , conexão Riemanniana \nabla$ e tensor curvatura $R$, e suponha também que o referencial $\left\{e_{A}\right\}_{A=1}^{m}$ seja adaptado a $M$, ou seja, os vetores $\left\{e_{i}\right\}_{i=1}^{n}$ são tangentes a $M$ quando restritos a $M$. Denote por $A_{\alpha}$ o operador forma associado ao campo normal $e_{\alpha}$, onde $n+1 \leq \alpha \leq m$. Segue que, ao longo de $M,\left\{\omega^{i}\right\}_{i=1}^{n}$ é base dual de $\left\{e_{i}\right\}_{i=1}^{n}$ e $\omega^{\alpha}=0$. A partir de agora só usaremos formas restritas a $M$.

Observe que, por (1.4), podemos escrever as formas de conexão de $\bar{M}$ em $M, \operatorname{com} n+1 \leq \alpha, \beta \leq$ $m$, da seguinte maneira:

$$
\begin{aligned}
\omega_{j}^{i}\left(e_{k}\right) & =\left\langle\bar{\nabla}_{e_{k}} e_{j}, e_{i}\right\rangle=\left\langle\left(\bar{\nabla}_{e_{k}} e_{j}\right)^{\top}, e_{i}\right\rangle=\left\langle\nabla_{e_{k}} e_{j}, e_{i}\right\rangle, \\
\omega_{j}^{\alpha}\left(e_{k}\right) & =\left\langle\bar{\nabla}_{e_{k}} e_{j}, e_{\alpha}\right\rangle=\left\langle\left(\bar{\nabla}_{e_{k}} e_{j}\right)^{\perp}, e_{\alpha}\right\rangle=\left\langle A_{\alpha}\left(e_{k}\right), e_{j}\right\rangle, \\
\omega_{\alpha}^{\beta}\left(e_{k}\right) & =\left\langle\bar{\nabla}_{e_{k}} e_{\alpha}, e_{\beta}\right\rangle=\left\langle\left(\bar{\nabla}_{e_{k}} e_{\alpha}\right)^{\perp}, e_{\beta}\right\rangle=\left\langle\nabla_{e_{k}}^{\perp} e_{\alpha}, e_{\beta}\right\rangle .
\end{aligned}
$$


Proposição 1.2.3. Valem as seguintes equações em $M$ :

$$
\begin{aligned}
& d \omega^{i}+\sum_{j} \omega_{j}^{i} \wedge \omega^{j}=0 . \\
& \sum_{p} \omega_{p}^{\alpha} \wedge \omega^{p}=0 \\
& d \omega_{j}^{i}+\sum_{p} \omega_{p}^{i} \wedge \omega_{j}^{p}=-\frac{1}{2} \sum_{k, l} R_{k l j}^{i} \omega^{k} \wedge \omega^{l}, \\
& d \omega_{j}^{\alpha}+\sum_{p} \omega_{p}^{\alpha} \wedge \omega_{j}^{p}=\frac{1}{2} \sum_{k, l}\left\langle\left(\nabla_{e_{k}} A_{\alpha}\right)\left(e_{l}\right)-\left(\nabla_{e_{l}} A_{\alpha}\right)\left(e_{k}\right), e_{j}\right\rangle, \\
& d \omega_{\alpha}^{\beta}+\sum_{\gamma} \omega_{\gamma}^{\beta} \wedge \omega_{\alpha}^{\gamma}=-\frac{1}{2} \sum_{k, l} R_{k l \alpha}^{\perp \beta} \omega^{k} \wedge \omega^{l} .
\end{aligned}
$$

Demonstração. Para provar (1.6), basta usar o fato de $\omega^{\alpha}=0$ em $M$, para $n+1 \leq \alpha \leq m$, na equação (1.5):

$$
0=d \omega^{i}+\sum_{B} \omega_{B}^{i} \wedge \omega^{B}=d \omega^{i}+\sum_{j} \omega_{j}^{i} \wedge \omega^{j}+\sum_{\alpha} \omega_{\alpha}^{i} \wedge \omega^{\alpha}=d \omega^{i}+\sum_{j} \omega_{j}^{i} \wedge \omega^{j} .
$$

Para (1.7), note que

$$
\begin{aligned}
\sum_{p} \omega_{p}^{\alpha} \wedge \omega^{p}\left(e_{k}, e_{l}\right) & =\omega_{l}^{\alpha}\left(e_{k}\right)-\omega_{k}^{\alpha}\left(e_{l}\right) \\
& =\left\langle A_{\alpha}\left(e_{k}\right), e_{l}\right\rangle-\left\langle A_{\alpha}\left(e_{l}\right), e_{k}\right\rangle \\
& =0
\end{aligned}
$$

pois $A_{\alpha}$ é simétrica.

Para (1.8), como $\omega_{j}^{i}=\sum_{k}\left\langle e_{i}, \nabla_{e_{k}} e_{j}\right\rangle \omega^{k}$, temos, por (1.6)

$$
\begin{aligned}
d \omega_{j}^{i} & =\sum_{k, l}\left(e_{l}\left\langle e_{i}, \nabla_{e_{k}} e_{j}\right\rangle\right) \omega^{l} \wedge \omega^{k}+\sum_{k}\left\langle e_{i}, \nabla_{e_{k}} e_{j}\right\rangle d \omega^{k} \\
& =\sum_{k, l}\left(\left\langle\nabla_{e_{l}} e_{i}, \nabla_{e_{k}} e_{j}\right\rangle+\left\langle e_{i}, \nabla_{e_{l}} \nabla_{e_{k}} e_{j}\right\rangle\right) \omega^{l} \wedge \omega^{k}-\sum_{k, l}\left\langle e_{i}, \nabla_{e_{k}} e_{j}\right\rangle \omega_{l}^{k} \wedge \omega^{l}
\end{aligned}
$$

Note que

$$
\begin{aligned}
\sum_{k, l}\left\langle e_{i}, \nabla_{e_{k}} e_{j}\right\rangle \omega_{l}^{k} \wedge \omega^{l} & =\sum_{k, l, q}\left\langle e_{i}, \nabla_{e_{k}} e_{j}\right\rangle\left\langle e_{k}, \nabla_{e_{q}} e_{l}\right\rangle \omega^{q} \wedge \omega^{l} \\
& =\sum_{l, q}\left\langle e_{i}, \sum_{k}\left\langle e_{k}, \nabla_{e_{q}} e_{l}\right\rangle \nabla_{e_{k}} e_{j}\right\rangle \omega^{q} \wedge \omega^{l} \\
& =\sum_{l, q}\left\langle e_{i}, \nabla_{\sum_{k}\left\langle e_{k}, \nabla_{e_{q}} e_{l}\right\rangle e_{k}} e_{j}\right\rangle \omega^{q} \wedge \omega^{l} \\
& =\sum_{l, q}\left\langle e_{i}, \nabla_{\nabla_{e_{q}} e_{l}} e_{j}\right\rangle \omega^{q} \wedge \omega^{l} .
\end{aligned}
$$


Além disso,

$$
\begin{aligned}
\sum_{p} \omega_{p}^{i} \wedge \omega_{j}^{p} & =\sum_{k, l, p}\left\langle e_{i}, \nabla_{e_{l}} e_{p}\right\rangle\left\langle e_{p}, \nabla_{e_{k}} e_{j}\right\rangle \omega^{l} \wedge \omega^{k} \\
& =-\sum_{k, l, p}\left\langle\nabla_{e_{l}} e_{i}, e_{p}\right\rangle\left\langle e_{p}, \nabla_{e_{k}} e_{j}\right\rangle \omega^{l} \wedge \omega^{k} \\
& =-\sum_{k, l}\left\langle\sum_{p}\left\langle\nabla_{e_{l}} e_{i}, e_{p}\right\rangle e_{p}, \nabla_{e_{k}} e_{j}\right\rangle \omega^{l} \wedge \omega^{k} \\
& =-\sum_{k, l}\left\langle\nabla_{e_{l}} e_{i}, \nabla_{e_{k}} e_{j}\right\rangle \omega^{l} \wedge \omega^{k}
\end{aligned}
$$

Logo obtemos a relação

$$
d \omega_{j}^{i}+\sum_{p} \omega_{p}^{i} \wedge \omega_{j}^{p}=\sum_{k, l}\left\langle e_{i}, \nabla_{e_{l}} \nabla_{e_{k}} e_{j}-\nabla_{\nabla_{e_{l}} e_{k}} e_{j}\right\rangle \omega^{l} \wedge \omega^{k} .
$$

Trocando os índices $k$ e $l$ obtemos

$$
d \omega_{j}^{i}+\sum_{p} \omega_{p}^{i} \wedge \omega_{j}^{p}=\sum_{k, l}\left\langle e_{i}, \nabla_{e_{k}} \nabla_{e_{l}} e_{j}-\nabla_{\nabla_{e_{k}} e_{l}} e_{j}\right\rangle \omega^{k} \wedge \omega^{l} .
$$

Somando as equações (1.11) e (1.12) com índices trocados, temos

$$
\begin{aligned}
2\left(d \omega_{j}^{i}+\sum_{p} \omega_{p}^{i} \wedge \omega_{j}^{p}\right) & =\sum_{k, l}\left\langle e_{i}, \nabla_{e_{l}} \nabla_{e_{k}} e_{j}-\nabla_{e_{k}} \nabla_{e_{l}} e_{j}+\nabla_{\nabla_{e_{k}} e_{l}} e_{j}-\nabla_{\nabla_{e_{l}} e_{k}} e_{j}\right\rangle \omega^{l} \wedge \omega^{k} \\
& =\sum_{k, l}\left\langle e_{i}, \nabla_{e_{l}} \nabla_{e_{k}} e_{j}-\nabla_{e_{k}} \nabla_{e_{l}} e_{j}-\nabla_{\left[e_{k}, e_{l}\right]} e_{j}\right\rangle \omega^{l} \wedge \omega^{k} \\
& =\sum_{k, l}\left\langle e_{i}, R\left(e_{k}, e_{l}\right) e_{j}\right\rangle \omega^{l} \wedge \omega^{k} \\
& =\sum_{k, l} R_{k l j}^{i} \omega^{l} \wedge \omega^{k} .
\end{aligned}
$$

Portanto

$$
d \omega_{j}^{i}+\sum_{p} \omega_{p}^{i} \wedge \omega_{j}^{p}=-\frac{1}{2} \sum_{k, l} R_{k l j}^{i} \omega^{k} \wedge \omega^{l}
$$

Agora, para (1.9), como $\omega_{j}^{\alpha}=\sum_{k}\left\langle A_{\alpha}\left(e_{k}\right), e_{j}\right\rangle \omega^{k}$, temos

$$
\begin{aligned}
d \omega_{j}^{\alpha} & =\sum_{k, l} e_{l}\left\langle A_{\alpha}\left(e_{k}\right), e_{j}\right\rangle \omega^{l} \wedge \omega^{k}+\sum_{k}\left\langle A_{\alpha}\left(e_{k}\right), e_{j}\right\rangle d \omega^{k} \\
& =\sum_{k, l}\left(\left\langle\nabla_{e_{l}} A_{\alpha}\left(e_{k}\right), e_{j}\right\rangle+\left\langle A_{\alpha}\left(e_{k}\right), \nabla_{e_{l}} e_{j}\right\rangle\right) \omega^{l} \wedge \omega^{k}-\sum_{k, l}\left\langle A_{\alpha}\left(e_{k}\right), e_{j}\right\rangle \omega_{l}^{k} \wedge \omega^{l} .
\end{aligned}
$$

Note que

$$
\begin{aligned}
\sum_{k, l}\left\langle A_{\alpha}\left(e_{k}\right), e_{j}\right\rangle \omega_{l}^{k} \wedge \omega^{l} & =\sum_{k, l, q}\left\langle A_{\alpha}\left(e_{k}\right), e_{j}\right\rangle\left\langle e_{k}, \nabla_{e_{q}} e_{l}\right\rangle \omega^{q} \wedge \omega^{l} \\
& =\sum_{l, q}\left\langle\sum_{k}\left\langle e_{k}, \nabla_{e_{q}} e_{l}\right\rangle e_{k}, A_{\alpha}\left(e_{j}\right)\right\rangle \omega^{q} \wedge \omega^{l} \\
& =\sum_{l, q}\left\langle A_{\alpha}\left(e_{j}\right), \nabla_{e_{q}} e_{l}\right\rangle \omega^{q} \wedge \omega^{l} .
\end{aligned}
$$


Além disso,

$$
\begin{aligned}
\sum_{p} \omega_{p}^{\alpha} \wedge \omega_{j}^{p} & =\sum_{k, l, p}\left\langle A_{\alpha}\left(e_{k}\right), e_{p}\right\rangle\left\langle e_{p}, \nabla_{e_{l}} e_{j}\right\rangle \omega^{k} \wedge \omega^{l} \\
& =\sum_{k, l}\left\langle A_{\alpha}\left(e_{k}\right), \sum_{p}\left\langle e_{p}, \nabla_{e_{l}} e_{j}\right\rangle e_{p}\right\rangle \omega^{k} \wedge \omega^{l} \\
& =\sum_{k, l}\left\langle A_{\alpha}\left(e_{k}\right), \nabla_{e_{l}} e_{j}\right\rangle \omega^{k} \wedge \omega^{l}
\end{aligned}
$$

Logo

$$
\begin{aligned}
d \omega_{j}^{\alpha}+\sum_{p} \omega_{p}^{\alpha} \wedge \omega_{j}^{p} & =\sum_{k, l}\left[\left\langle\nabla_{e_{l}} A_{\alpha}\left(e_{k}\right), e_{j}\right\rangle+\left\langle A_{\alpha}\left(e_{j}\right), \nabla_{e_{l}} e_{k}\right\rangle\right] \omega^{l} \wedge \omega^{k} \\
& =\sum_{k, l}\left\langle\nabla_{e_{l}} A_{\alpha}\left(e_{k}\right)-A_{\alpha}\left(\nabla_{e_{l}} e_{k}\right), e_{j}\right\rangle \omega^{l} \wedge \omega^{k} \\
& =\sum_{k, l}\left\langle\left(\nabla_{e_{l}} A_{\alpha}\right)\left(e_{k}\right), e_{j}\right\rangle \omega^{l} \wedge \omega^{k}
\end{aligned}
$$

Trocando os índices $k$ e $l$ em (1.13) e somando as equações com índices trocados como anteriormente obtemos

$$
d \omega_{j}^{\alpha}+\sum_{p} \omega_{p}^{\alpha} \wedge \omega_{j}^{p}=\frac{1}{2} \sum_{k, l}\left\langle\left(\nabla_{e_{k}} A_{\alpha}\right)\left(e_{l}\right)-\left(\nabla_{e_{l}} A_{\alpha}\right)\left(e_{k}\right), e_{j}\right\rangle \omega^{k} \wedge \omega^{l}
$$

A demonstração de (1.10) é análoga à de (1.8). Como $\omega_{\alpha}^{\beta}=\sum_{\gamma}\left\langle e_{\beta}, \nabla_{e_{k}}^{\perp} e_{\alpha}\right\rangle \omega^{k}$, segue que

$$
d \omega_{\alpha}^{\beta}=\sum_{k, l}\left[\left\langle\nabla_{e_{l}}^{\perp} e_{\beta}, \nabla_{e_{k}}^{\perp} e_{\alpha}\right\rangle+\left\langle e_{\beta}, \nabla_{e_{l}}^{\perp} \nabla_{e_{k}}^{\perp} e_{\alpha}\right\rangle\right] \omega^{l} \wedge \omega^{k}-\sum_{k, l}\left\langle e_{\beta}, \nabla_{e_{k}}^{\perp} e_{\alpha}\right\rangle \omega_{l}^{k} \wedge \omega^{l}
$$

Temos

$$
\sum_{k, l}\left\langle e_{\beta}, \nabla_{e_{k}}^{\perp} e_{\alpha}\right\rangle \omega_{l}^{k} \wedge \omega^{l}=\sum_{l, q}\left\langle e_{\beta}, \nabla_{\nabla_{e_{q}}^{\perp} e_{l}}^{\perp} e_{\alpha}\right\rangle \omega^{q} \wedge \omega^{l}
$$

e

$$
\sum_{\gamma} \omega_{\gamma}^{\beta} \wedge \omega_{\alpha}^{\gamma}=-\sum_{k, l}\left\langle\nabla_{e_{l}}^{\perp} e_{\beta}, \nabla_{e_{k}}^{\perp} e_{\alpha}\right\rangle \omega^{l} \wedge \omega^{k}
$$

Logo

$$
d \omega_{\alpha}^{\beta}+\sum_{\gamma} \omega_{\gamma}^{\beta} \wedge \omega_{\alpha}^{\gamma}=\sum_{k, l}\left\langle e_{\beta}, \nabla_{e_{l}}^{\perp} \nabla_{e_{k}}^{\perp} e_{\alpha}-\nabla_{\nabla_{e_{l}}^{\perp} e_{k}}^{\perp} e_{\alpha}\right\rangle \omega^{l} \wedge \omega^{k} .
$$

Trocando os índices $k$ e $l$ e somando as equações obtemos

$$
d \omega_{\alpha}^{\beta}+\sum_{\gamma} \omega_{\gamma}^{\beta} \wedge \omega_{\alpha}^{\gamma}=-\frac{1}{2} \sum_{k} \sum_{l} R_{k l \alpha}^{\perp \beta} \omega^{k} \wedge \omega^{l}
$$

\subsection{Subvariedades de $\mathbb{S}^{m} \times \mathbb{R}$ e $\mathbb{H}^{m} \times \mathbb{R}$}

Denote por $\mathbb{L}^{r}$ o espaço de Lorentz, ou seja, $\mathbb{R}^{r}$ munido da forma quadrática

$$
-\left(d x^{0}\right)^{2}+\left(d x^{1}\right)^{2}+\ldots+\left(d x^{r-1}\right)^{2} .
$$


Considere

$$
\mathbb{Q}_{1}^{m}=\mathbb{S}^{m}=\left\{\left(x^{0}, \ldots, x^{m}\right) \in \mathbb{R}^{m+1} ;\left(x^{0}\right)^{2}+\left(x^{1}\right)^{2}+\ldots+\left(x^{m}\right)^{2}=1\right\}
$$

e

$$
\mathbb{Q}_{-1}^{m}=\mathbb{H}^{m}=\left\{\left(x^{0}, \ldots, x^{m}\right) \in \mathbb{L}^{m+1} ;-\left(x^{0}\right)^{2}+\left(x^{1}\right)^{2}+\ldots+\left(x^{m}\right)^{2}=-1, x^{0}>0\right\},
$$

ou seja, $\mathbb{Q}_{\varepsilon}^{m}$ denota a esfera $\mathbb{S}^{m}$ se $\varepsilon=1$ ou o espaço hiperbólico $\mathbb{H}^{m}$ se $\varepsilon=-1$.

Denotaremos por $\mathbb{E}^{r}$ o espaço Euclidiano $\mathbb{R}^{r}$ quando $\varepsilon=1$ ou o espaço de Lorentz $\mathbb{L}^{r}$ quando $\varepsilon=-1$, e denotaremos por $\langle,\rangle_{\varepsilon}$ a métrica semi-riemanniana em $\mathbb{E}^{r}$.

Consideramos também o produto $\mathbb{Q}_{\varepsilon}^{m} \times \mathbb{R}$. Desta maneira temos a inclusão

$$
\mathbb{Q}_{\varepsilon}^{m} \times \mathbb{R} \subset \mathbb{E}^{m+1} \times \mathbb{R}=\mathbb{E}^{m+2},
$$

e portanto $\mathbb{Q}_{\varepsilon}^{m} \times \mathbb{R}$ é subvariedade de $\mathbb{E}^{m+2}$, de codimensão 1 , munida da métrica induzida pela inclusão, que também denotaremos por $\langle,\rangle_{\varepsilon}$. Denotaremos por $\frac{\partial}{\partial t}$ o vetor unitário dando a orientação de $\mathbb{R}$ em $\mathbb{Q}_{\varepsilon}^{m} \times \mathbb{R}$, que chamaremos de vetor vertical.

Qualquer campo vetorial em $\mathbb{Q}_{\varepsilon}^{m} \times \mathbb{R}$ pode ser escrito (localmente) como $X(r, t)=X_{\mathbb{Q}}^{t}(r)+X_{\mathbb{R}}^{r}(t)$, onde para cada $t \in \mathbb{R}, X_{\mathbb{Q}}^{t}$ é um campo vetorial em $\mathbb{Q}_{\varepsilon}^{m}$, e para cada $r \in \mathbb{Q}_{\varepsilon}^{m}, X_{\mathbb{R}}^{r}$ é um campo vetorial em $\mathbb{R}$. Assim, podemos escrever $X=X_{\mathbb{Q}}+X_{\mathbb{R}}=X_{\mathbb{Q}}+\left\langle X, \frac{\partial}{\partial t}\right\rangle_{\varepsilon} \frac{\partial}{\partial t}$. Observe que como $\mathbb{Q}_{\varepsilon}^{m} \subset \mathbb{E}^{m+1}$ é subvariedade de $\mathbb{E}^{m+1}$, a métrica $\langle,\rangle_{\varepsilon}$ coincide com a métrica produto, ou seja, $\langle,\rangle_{\varepsilon}=\langle,\rangle_{\mathbb{Q}} \oplus\langle,\rangle_{\mathbb{R}}$, onde $\langle,\rangle_{\mathbb{Q}}$ denota a métrica em $\mathbb{Q}_{\varepsilon}^{m}$ induzida pela inclusão em $\mathbb{E}^{m+1}$ e $\langle,\rangle_{\mathbb{R}}$ denota a métrica canônica em $\mathbb{R}$. Vamos denotar por $\overline{\bar{\nabla}}$ e $\bar{\nabla}$ as conexões de $\mathbb{E}^{m+2}$ e de $\mathbb{Q}_{\varepsilon}^{m} \times \mathbb{R}$, respectivamente. Então, se $X, Y \in \Gamma\left(\mathbb{Q}_{\varepsilon}^{m} \times \mathbb{R}\right)$, temos $\bar{\nabla}_{Y} X=\nabla_{Y_{\mathbb{Q}}}^{\mathbb{Q}} X_{\mathbb{M}}+\nabla_{Y_{\mathbb{R}}}^{\mathbb{R}} X_{\mathbb{R}}$. Seja também $\bar{R}$ o tensor curvatura de $\mathbb{Q}_{\varepsilon}^{m} \times \mathbb{R}$.

Seja $x \in \mathbb{Q}_{\varepsilon}^{m} \times \mathbb{R}$. Escrevendo $x=\left(x^{0}, \ldots, x^{m+1}\right)$, temos que $p=\left(x^{0}, \ldots, x^{m}\right) \in \mathbb{Q}_{\varepsilon}^{m}$, ou seja, $\varepsilon\left(x^{0}\right)^{2}+\left(x^{1}\right)^{2}+\ldots+\left(x^{m}\right)^{2}=\varepsilon$. Derivando esta expressão, obtemos $\varepsilon x^{0} d x^{0}+x^{1} d x^{1}+\ldots+x^{m} d x^{m}=0$, e portanto se $v_{\mathbb{Q}} \in T_{p} \mathbb{Q}_{\varepsilon}^{m}$, entao $\left\langle p, v_{\mathbb{Q}}\right\rangle=0$. Seja $\bar{N}$ o campo vetorial em $x \in \mathbb{Q}_{\varepsilon}^{m} \times \mathbb{R}$ definido por

$$
\bar{N}(x)=\left(x^{0}, \ldots, x^{m}, 0\right) .
$$

Então pela expressão acima temos que, para todo $v \in T_{x}\left(\mathbb{Q}_{\varepsilon}^{m} \times \mathbb{R}\right)$,

$$
\langle\bar{N}(x), v\rangle_{\varepsilon}=\left\langle\left(x^{0}, \ldots, x^{m}\right), v_{\mathbb{Q}}\right\rangle_{\mathbb{Q}}+\left\langle 0, v_{\mathbb{R}}\right\rangle_{\mathbb{R}}=0,
$$

ou seja, $\bar{N}(x)$ é o campo normal a $\mathbb{Q}_{\varepsilon}^{m} \times \mathbb{R}$ em $\mathbb{E}^{m+2}$ no ponto $x \in \mathbb{Q}_{\varepsilon}^{m} \times \mathbb{R}$. Definimos o operador forma $A_{\bar{N}}$ de $\mathbb{Q}_{\varepsilon}^{m} \times \mathbb{R}$ associado à $\bar{N}$ por

$$
\overline{\bar{\nabla}}_{X} Y=\bar{\nabla}_{X} Y+\left\langle A_{\bar{N}}(X), Y\right\rangle_{\varepsilon} \bar{N}
$$

onde $X, Y \in \Gamma\left(\mathbb{Q}_{\varepsilon}^{m} \times \mathbb{R}\right)$, ou seja,

$$
\left\langle A_{\bar{N}}(X), Y\right\rangle_{\varepsilon}=\varepsilon\left\langle\overline{\bar{\nabla}}_{X} Y, \bar{N}\right\rangle_{\varepsilon}
$$

pois $\langle\bar{N}, \bar{N}\rangle_{\varepsilon}=\varepsilon$. Desta forma temos que $\left\langle A_{\bar{N}}(X), Y\right\rangle_{\varepsilon}=-\varepsilon\left\langle Y, \bar{\nabla}_{X} \bar{N}\right\rangle_{\varepsilon}$ e, portanto, $A_{\bar{N}}(X)=$ $-\varepsilon d \bar{N}(X)=-\varepsilon X_{\mathbb{Q}}=-\varepsilon X+\varepsilon\left\langle X, \frac{\partial}{\partial t}\right\rangle_{\varepsilon} \frac{\partial}{\partial t}$.

A partir de agora, caso não seja mencionado o contrário, faremos a seguinte convenção de índices:

$$
\begin{gathered}
0 \leq A, B, C \ldots \leq m+1, \\
1 \leq i, j, k \ldots \leq n, \\
n+1 \leq \alpha, \beta, \gamma \ldots \leq m+1 .
\end{gathered}
$$

Seja $M$ uma subvariedade Riemanniana de dimensão $n$ de $\mathbb{Q}_{\varepsilon}^{m} \times \mathbb{R}$, com métrica riemanniana induzida pela inclusão, conexão Riemanniana $\nabla$ e tensor curvatura $R$. Considere um referencial 
ortonormal local $\left\{e_{C}\right\}_{C=1}^{m+1}$ em $\mathbb{Q}_{\varepsilon}^{m} \times \mathbb{R}$ adaptado a $M$ e denote por $A_{\alpha}$ o operador forma associado ao campo normal $e_{\alpha}$. Tome a base dual $\left\{\omega^{C}\right\}_{C=1}^{m+1}$ e as formas de conexão $\left\{\omega_{B}^{A}\right\}_{A, B=1}^{m+1}$ de $\mathbb{Q}_{\varepsilon}^{m} \times \mathbb{R}$ no referencial $\left\{e_{C}\right\}_{C=1}^{m+1}$. No que segue só usaremos formas restritas a $M$. Definimos em $M$ o campo vetorial $e_{0}=\bar{N}$ e as 1 -formas

$$
\begin{gathered}
\omega_{C}^{0}\left(e_{k}\right)=\left\langle A_{\bar{N}}\left(e_{k}\right), e_{C}\right\rangle_{\varepsilon}=-\varepsilon\left\langle e_{k}, e_{C}\right\rangle_{\varepsilon}+\varepsilon\left\langle e_{k}, \frac{\partial}{\partial t}\right\rangle_{\varepsilon}\left\langle e_{C}, \frac{\partial}{\partial t}\right\rangle_{\varepsilon}, \\
\omega_{0}^{C}=-\varepsilon \omega_{C}^{0} .
\end{gathered}
$$

Lema 1.3.1. Com as definições acima temos que

$$
\overline{\bar{\nabla}}_{e_{k}} e_{C}=\sum_{B} \omega_{C}^{B}\left(e_{k}\right) e_{B}
$$

Demonstração. Primeiramente observe que

$$
\begin{aligned}
\left\langle\overline{\bar{\nabla}}_{e_{k}} e_{C}, e_{0}\right\rangle_{\varepsilon} & =\left\langle\left\langle A_{\bar{N}}\left(e_{k}\right), e_{C}\right\rangle_{\varepsilon} e_{0}, e_{0}\right\rangle_{\varepsilon} \\
& =\varepsilon\left\langle A_{\bar{N}}\left(e_{k}\right), e_{C}\right\rangle_{\varepsilon} \\
& =\varepsilon \omega_{C}^{0}\left(e_{k}\right)
\end{aligned}
$$

Agora, para $1 \leq B \leq m+1$, temos

$$
\begin{aligned}
\left\langle\overline{\bar{\nabla}}_{e_{k}} e_{0}, e_{B}\right\rangle_{\varepsilon} & =-\left\langle\overline{\bar{\nabla}}_{e_{k}} e_{B}, e_{0}\right\rangle_{\varepsilon} \\
& =-\varepsilon \omega_{B}^{0}\left(e_{k}\right) \\
& =\omega_{0}^{B}\left(e_{k}\right),
\end{aligned}
$$

e se $1 \leq C \leq m+1$, por (1.4) temos

$$
\begin{aligned}
\left\langle\bar{\nabla}_{e_{k}} e_{C}, e_{B}\right\rangle_{\varepsilon} & =\left\langle\bar{\nabla}_{e_{k}} e_{C}, e_{B}\right\rangle_{\varepsilon} \\
& =\omega_{C}^{B}\left(e_{k}\right) .
\end{aligned}
$$

Seja $\left\{E_{B}\right\}_{B=0}^{m+1}$ o referencial canônico de $\mathbb{E}^{m+2}$, com $\left\langle E_{0}, E_{0}\right\rangle_{\varepsilon}=\varepsilon$ e $E_{m+1}=\frac{\partial}{\partial t}$. Seja $P \in$ $\mathcal{M}_{m+2}(\mathbb{R})$ a matriz cujas colunas são as coordenadas de $\left(e_{C}\right)$ no referencial $\left(E_{B}\right)$, com índices variando de 0 a $m+1$, ou seja,

$$
e_{C}=\sum_{B} P_{C}^{B} E_{B}
$$

e vamos supor que $\left(e_{C}\right)$ é uma base positiva com relação à $\left(E_{B}\right)$, ou seja, $\operatorname{det} P=1$. Então temos que

$$
\overline{\bar{\nabla}}_{e_{k}} e_{C}=\overline{\bar{\nabla}}_{e_{k}} \sum_{B} P_{C}^{B} E_{B}=\sum_{B} d P_{C}^{B}\left(e_{k}\right) E_{B}
$$

Por outro lado, utilizando o lema 1.3.1 acima temos

$$
\overline{\bar{\nabla}}_{e_{k}} e_{C}=\sum_{D} \omega_{C}^{D}\left(e_{k}\right) e_{D}=\sum_{D} \omega_{C}^{D}\left(e_{k}\right) \sum_{B} P_{D}^{B} E_{B}=\sum_{B}\left(\sum_{D} P_{D}^{B} \omega_{C}^{D}\left(e_{k}\right)\right) E_{B}
$$

Portanto vale que

$$
d P_{C}^{B}\left(e_{k}\right)=\sum_{D} P_{D}^{B} \omega_{C}^{D}\left(e_{k}\right),
$$

ou seja, se $\Omega=\left(\omega_{C}^{B}\right) \in \mathcal{M}_{m+2}(\mathbb{R})$, então

$$
d P=P \Omega,
$$


ou seja,

$$
P^{-1} d P=\Omega .
$$

Observação 1.3.2. Os elementos da primeira coluna de $P$ são as coordenadas de $\bar{N}$, ou seja, $\left(P_{0}^{0}, \ldots, P_{0}^{m}\right)$ é a componente do vetor posição de $M$ no fator $\mathbb{Q}_{\varepsilon}^{m}$.

Seja $G=\operatorname{diag}(\varepsilon, 1, \ldots, 1) \in \mathcal{M}_{m+2}(\mathbb{R})$. Tome o grupo de Lie

$$
S O\left(\mathbb{E}^{m+2}\right)=\left\{Z \in \mathcal{M}_{m+2}(\mathbb{R}) ; Z^{t} G Z=G, \operatorname{det} Z=1\right\}
$$

e sua álgebra de Lie

$$
\mathfrak{s o}\left(\mathbb{E}^{m+2}\right)=\left\{H \in \mathcal{M}_{m+2}(\mathbb{R}) ; H^{t} G+G H=0\right\} .
$$

Denote por $S O^{+}\left(\mathbb{E}^{m+2}\right)$ a componente conexa da identidade $I$ em $S O\left(\mathbb{E}^{m+2}\right)$. Como

$$
\begin{aligned}
G_{B}^{A}=\left\langle e_{A}, e_{B}\right\rangle_{\varepsilon} & =\left\langle\sum_{C} P_{A}^{C} E_{C}, \sum_{D} P_{B}^{D} E_{D}\right\rangle_{\varepsilon} \\
& =\sum_{C, D} P_{A}^{C} P_{B}^{D}\left\langle E_{C}, E_{D}\right\rangle_{\varepsilon} \\
& =\sum_{C, D} P_{A}^{C} P_{B}^{D} G_{D}^{C} \\
& =\sum_{C, D} P_{A}^{C} G_{D}^{C} P_{B}^{D},
\end{aligned}
$$

ou seja, $P^{-1} G P=G$, $\operatorname{logo} P \in S O^{+}\left(\mathbb{E}^{m+2}\right)$, pois $\operatorname{det} P=1$. Além disso, como

$$
\begin{array}{ll} 
& P^{t} G P=G \\
\Rightarrow & P G P^{t}=G \\
\Rightarrow & P G d P^{t}+d P G P^{t}=0 \\
\Rightarrow & G d P^{t}\left(P^{-1}\right)^{t}+P^{-1} d P G=0 \\
\Rightarrow & d P^{t}\left(P^{-1}\right)^{t} G+G P^{-1} d P=0 \\
\Rightarrow & \Omega^{t} G+G \Omega=0,
\end{array}
$$

$\operatorname{Logo} \Omega \in \mathfrak{s o}\left(\mathbb{E}^{m+2}\right)$.

Vamos agora desenvolver as equações fundamentais que são satisfeitas quando $M$ é subvariedade de $\mathbb{Q}_{\varepsilon}^{m} \times \mathbb{R}$. Para cada índice $\alpha$ definimos as funções

$$
\nu^{\alpha}=\left\langle e_{\alpha}, \frac{\partial}{\partial t}\right\rangle_{\varepsilon}
$$

em $M$ e denotaremos por $T$ a projeção de $\frac{\partial}{\partial t}$ em $T M$. Desta forma podemos escrever

$$
\frac{\partial}{\partial t}=T+\sum_{\alpha} \nu^{\alpha} e_{\alpha}
$$


Proposição 1.3.3. Para $X, Y, Z, W \in \Gamma(T M)$ temos

$$
\begin{aligned}
\langle\bar{R}(X, Y) Z, W\rangle_{\varepsilon}= & {\left[\langle X, Z\rangle_{\varepsilon}\langle Y, W\rangle_{\varepsilon}-\langle Y, Z\rangle_{\varepsilon}\langle X, W\rangle_{\varepsilon}\right.} \\
& -\langle Y, T\rangle_{\varepsilon}\langle W, T\rangle_{\varepsilon}\langle X, Z\rangle_{\varepsilon}-\langle X, T\rangle_{\varepsilon}\langle Z, T\rangle_{\varepsilon}\langle Y, W\rangle_{\varepsilon} \\
& \left.+\langle X, T\rangle_{\varepsilon}\langle W, T\rangle_{\varepsilon}\langle Y, Z\rangle_{\varepsilon}+\langle Y, T\rangle_{\varepsilon}\langle Z, T\rangle_{\varepsilon}\langle X, W\rangle_{\varepsilon}\right], \\
\left\langle\bar{R}(X, Y) e_{\alpha}, Z\right\rangle_{\varepsilon}= & \varepsilon \nu^{\alpha}\left[\langle X, Z\rangle_{\varepsilon}\langle Y, T\rangle_{\varepsilon}-\langle Y, Z\rangle_{\varepsilon}\langle X, T\rangle_{\varepsilon}\right], \\
\left\langle\bar{R}(X, Y) e_{\alpha}, e_{\beta}\right\rangle_{\varepsilon}= & 0 .
\end{aligned}
$$

Demonstração. Para todo $X, Y, Z, W \in \Gamma\left(\mathbb{Q}_{\varepsilon}^{m} \times \mathbb{R}\right)$ temos:

$$
\begin{aligned}
\langle\bar{R}(X, Y) Z, W\rangle_{\varepsilon} & =\left\langle\bar{\nabla}_{Y} \bar{\nabla}_{X} Z-\bar{\nabla}_{X} \bar{\nabla}_{Y} Z+\bar{\nabla}_{[X, Y]} Z, W\right\rangle_{\varepsilon} \\
& =\left\langle R_{\mathbb{Q}}\left(X_{\mathbb{Q}}, Y_{\mathbb{Q}}\right) Z_{\mathbb{Q}}+R_{\mathbb{R}}\left(X_{\mathbb{R}}, Y_{\mathbb{R}}\right) Z_{\mathbb{R}}, W_{\mathbb{Q}}+W_{\mathbb{R}}\right\rangle_{\varepsilon} \\
& =\left\langle R_{\mathbb{Q}}\left(X_{\mathbb{Q}}, Y_{\mathbb{Q}}\right) Z_{\mathbb{Q}}, W_{\mathbb{Q}}\right\rangle_{\mathbb{Q}}+\left\langle R_{\mathbb{R}}\left(X_{\mathbb{R}}, Y_{\mathbb{R}}\right) Z_{\mathbb{R}}, W_{\mathbb{R}}\right\rangle_{\mathbb{R}} .
\end{aligned}
$$

Sabemos que

$$
\left\langle R_{\mathbb{R}}\left(X_{\mathbb{R}}, Y_{\mathbb{R}}\right) Z_{\mathbb{R}}, W_{\mathbb{R}}\right\rangle_{\mathbb{R}}=0 .
$$

Além disso, como $\mathbb{Q}_{\varepsilon}^{m}$ tem curvatura seccional constante igual a $\varepsilon$, temos

$$
\left\langle R_{\mathbb{Q}}\left(X_{\mathbb{Q}}, Y_{\mathbb{Q}}\right) Z_{\mathbb{Q}}, W_{\mathbb{Q}}\right\rangle_{\mathbb{Q}}=\varepsilon\left[\left\langle X_{\mathbb{Q}}, Z_{\mathbb{Q}}\right\rangle_{\mathbb{Q}}\left\langle Y_{\mathbb{Q}}, W_{\mathbb{Q}}\right\rangle_{\mathbb{Q}}-\left\langle Y_{\mathbb{Q}}, Z_{\mathbb{Q}}\right\rangle_{\mathbb{Q}}\left\langle X_{\mathbb{Q}}, W_{\mathbb{Q}}\right\rangle_{\mathbb{Q}}\right] .
$$

Como $\forall X, Z \in \Gamma\left(\mathbb{Q}_{\varepsilon}^{m} \times \mathbb{R}\right)$ vale

$$
\left\langle X_{\mathbb{Q}}, Z_{\mathbb{Q}}\right\rangle_{\mathbb{Q}}=\left\langle X_{\mathbb{Q}}, Z_{\mathbb{Q}}\right\rangle_{\varepsilon}
$$

segue que

$$
\langle\bar{R}(X, Y) Z, W\rangle_{\varepsilon}=\varepsilon\left[\left\langle X_{\mathbb{Q}}, Z_{\mathbb{Q}}\right\rangle_{\varepsilon}\left\langle Y_{\mathbb{Q}}, W_{\mathbb{Q}}\right\rangle_{\varepsilon}-\left\langle Y_{\mathbb{Q}}, Z_{\mathbb{Q}}\right\rangle_{\varepsilon}\left\langle X_{\mathbb{Q}}, W_{\mathbb{Q}}\right\rangle_{\varepsilon}\right] .
$$

Observe agora que se $X \in \Gamma(T M)$, então

$$
\begin{aligned}
\left\langle X, \frac{\partial}{\partial t}\right\rangle_{\varepsilon} & =\left\langle X, \frac{\partial}{\partial t}\right\rangle_{\varepsilon}-\left\langle X, \sum_{\alpha} \nu^{\alpha} e_{\alpha}\right\rangle_{\varepsilon} \\
& =\left\langle X, \frac{\partial}{\partial t}-\sum_{\alpha} \nu^{\alpha} e_{\alpha}\right\rangle_{\varepsilon} \\
& =\langle X, T\rangle_{\varepsilon},
\end{aligned}
$$

$\log 0$

$$
\begin{aligned}
X_{\mathbb{Q}} & =X-\left\langle X, \frac{\partial}{\partial t}\right\rangle_{\varepsilon} \frac{\partial}{\partial t} \\
& =X-\langle X, T\rangle_{\varepsilon} \frac{\partial}{\partial t},
\end{aligned}
$$

e valem expressões similares para $Y, Z, W \in \Gamma(T M)$. Note que

$$
\begin{aligned}
\left\langle X_{\mathbb{Q}}, Z_{\mathbb{Q}}\right\rangle \varepsilon & =\left\langle X-\langle X, T\rangle_{\varepsilon} \frac{\partial}{\partial t}, Z-\langle Z, T\rangle_{\varepsilon} \frac{\partial}{\partial t}\right\rangle_{\varepsilon} \\
& =\langle X, Z\rangle_{\varepsilon}-\langle X, T\rangle_{\varepsilon}\left\langle Z, \frac{\partial}{\partial t}\right\rangle_{\varepsilon}-\langle Z, T\rangle_{\varepsilon}\left\langle X, \frac{\partial}{\partial t}\right\rangle_{\varepsilon}+\langle X, T\rangle_{\varepsilon}\langle Z, T\rangle_{\varepsilon} \\
& =\langle X, Z\rangle_{\varepsilon}-\langle X, T\rangle_{\varepsilon}\langle Z, T\rangle_{\varepsilon} .
\end{aligned}
$$


Portanto, usando a expressão acima, segue que

$$
\begin{aligned}
\langle\bar{R}(X, Y) Z, W\rangle_{\varepsilon}= & \varepsilon\left[\left\langle X_{\mathbb{Q}}, Z_{\mathbb{Q}}\right\rangle_{\varepsilon}\left\langle Y_{\mathbb{Q}}, W_{\mathbb{Q}}\right\rangle_{\varepsilon}-\left\langle Y_{\mathbb{Q}}, Z_{\mathbb{Q}}\right\rangle_{\varepsilon}\left\langle X_{\mathbb{Q}}, W_{\mathbb{Q}}\right\rangle_{\varepsilon}\right] \\
= & \varepsilon\left[\left[\langle, Z\rangle_{\varepsilon}-\langle X, T\rangle_{\varepsilon}\langle Z, T\rangle_{\varepsilon}\right]\left[\langle Y, W\rangle_{\varepsilon}-\langle Y, T\rangle_{\varepsilon}\langle W, T\rangle_{\varepsilon}\right]\right. \\
& \left.-\left[\langle Y, Z\rangle_{\varepsilon}-\langle Y, T\rangle_{\varepsilon}\langle Z, T\rangle_{\varepsilon}\right]\left[\langle X, W\rangle_{\varepsilon}-\langle X, T\rangle_{\varepsilon}\langle W, T\rangle_{\varepsilon}\right]\right] \\
= & \varepsilon\left[\langle X, Z\rangle_{\varepsilon}\langle Y, W\rangle_{\varepsilon}-\langle Y, Z\rangle_{\varepsilon}\langle X, W\rangle_{\varepsilon}\right. \\
& -\langle Y, T\rangle_{\varepsilon}\langle W, T\rangle_{\varepsilon}\langle X, Z\rangle_{\varepsilon}-\langle X, T\rangle_{\varepsilon}\langle Z, T\rangle_{\varepsilon}\langle Y, W\rangle_{\varepsilon} \\
& \left.+\langle X, T\rangle_{\varepsilon}\langle W, T\rangle_{\varepsilon}\langle Y, Z\rangle_{\varepsilon}+\langle Y, T\rangle_{\varepsilon}\langle Z, T\rangle_{\varepsilon}\langle X, W\rangle_{\varepsilon}\right],
\end{aligned}
$$

e temos a equação (1.14).

Escrevendo $\left(e_{\alpha}\right)_{\mathbb{Q}}=e_{\alpha}-\nu^{\alpha} \frac{\partial}{\partial t}$ e usando o fato que

$$
\begin{aligned}
\left\langle X_{\mathbb{Q}},\left(e_{\alpha}\right)_{\mathbb{Q}}\right\rangle_{\varepsilon} & =\left\langle X-\langle X, T\rangle_{\varepsilon} \frac{\partial}{\partial t}, e_{\alpha}-\nu^{\alpha} \frac{\partial}{\partial t}\right\rangle_{\varepsilon} \\
& =\left\langle X, e_{\alpha}\right\rangle_{\varepsilon}-\nu^{\alpha}\langle X, T\rangle_{\varepsilon}-\nu^{\alpha}\left\langle X, \frac{\partial}{\partial t}\right\rangle_{\varepsilon}+\nu^{\alpha}\langle X, T\rangle_{\varepsilon} \\
& =-\nu^{\alpha}\langle X, T\rangle_{\varepsilon},
\end{aligned}
$$

segue que

$$
\begin{aligned}
\left\langle\bar{R}(X, Y) e_{\alpha}, Z\right\rangle_{\varepsilon}= & \varepsilon\left[\left\langle X_{\mathbb{Q}},\left(e_{\alpha}\right)_{\mathbb{Q}}\right\rangle_{\varepsilon}\left\langle Y_{\mathbb{Q}}, Z_{\mathbb{Q}}\right\rangle_{\varepsilon}-\left\langle Y_{\mathbb{Q}},\left(e_{\alpha}\right)_{\mathbb{Q}}\right\rangle_{\varepsilon}\left\langle X_{\mathbb{Q}}, Z_{\mathbb{Q}}\right\rangle_{\varepsilon}\right] \\
= & \varepsilon\left[-\nu^{\alpha}\langle X, T\rangle_{\varepsilon}\left[\langle Y, Z\rangle_{\varepsilon}-\langle Y, T\rangle_{\varepsilon}\langle Z, T\rangle_{\varepsilon}\right]\right. \\
& \left.+\nu^{\alpha}\langle Y, T\rangle_{\varepsilon}\left[\langle X, Z\rangle_{\varepsilon}-\langle X, T\rangle_{\varepsilon}\langle Z, T\rangle_{\varepsilon}\right]\right] \\
= & \varepsilon \nu^{\alpha}\left[\langle Y, T\rangle_{\varepsilon}\langle X, Z\rangle_{\varepsilon}-\langle X, T\rangle_{\varepsilon}\langle Y, Z\rangle_{\varepsilon}\right],
\end{aligned}
$$

e temos a equação (1.15).

Além disso,

$$
\begin{aligned}
\left\langle\bar{R}(X, Y) e_{\alpha}, e_{\beta}\right\rangle_{\varepsilon} & =\varepsilon\left[\left\langle X_{\mathbb{Q}},\left(e_{\alpha}\right)_{\mathbb{Q}}\right\rangle_{\varepsilon}\left\langle Y_{\mathbb{Q}},\left(e_{\beta}\right)_{\mathbb{Q}}\right\rangle_{\varepsilon}-\left\langle Y_{\mathbb{Q}},\left(e_{\alpha}\right)_{\mathbb{Q}}\right\rangle_{\varepsilon}\left\langle X_{\mathbb{Q}},\left(e_{\beta}\right)_{\mathbb{Q}}\right\rangle_{\varepsilon}\right] \\
& =\varepsilon\left[\left(-\nu^{\alpha}\langle X, T\rangle_{\varepsilon}\right)\left(-\nu^{\beta}\langle Y, T\rangle_{\varepsilon}\right)-\left(-\nu^{\alpha}\langle Y, T\rangle_{\varepsilon}\right)\left(-\nu^{\beta}\langle X, T\rangle_{\varepsilon}\right)\right] \\
& =0
\end{aligned}
$$

e temos a equação (1.16).

Corolário 1.3.4. Para $X, Y, Z, W \in \Gamma(T M)$, as equações de Gauss, Codazzi e Ricci podem ser reescritas respectivamente como

$$
\begin{aligned}
\langle R(X, Y) Z, W\rangle_{\varepsilon}= & \sum_{\gamma}\left[\left\langle A_{\gamma} X, Z\right\rangle_{\varepsilon}\left\langle A_{\gamma} Y, W\right\rangle_{\varepsilon}-\left\langle A_{\gamma} Y, Z\right\rangle_{\varepsilon}\left\langle A_{\gamma} X, W\right\rangle_{\varepsilon}\right] \\
& +\varepsilon\left[\langle X, Z\rangle_{\varepsilon}\langle Y, W\rangle_{\varepsilon}-\langle Y, Z\rangle_{\varepsilon}\langle X, W\rangle_{\varepsilon}\right. \\
& -\langle Y, T\rangle_{\varepsilon}\langle W, T\rangle_{\varepsilon}\langle X, Z\rangle_{\varepsilon}-\langle X, T\rangle_{\varepsilon}\langle Z, T\rangle_{\varepsilon}\langle Y, W\rangle_{\varepsilon} \\
& \left.+\langle X, T\rangle_{\varepsilon}\langle W, T\rangle_{\varepsilon}\langle Y, Z\rangle_{\varepsilon}+\langle Y, T\rangle_{\varepsilon}\langle Z, T\rangle_{\varepsilon}\langle X, W\rangle_{\varepsilon}\right], \\
\varepsilon \nu^{\alpha}\left[\langle Y, T\rangle_{\varepsilon} X-\langle X, T\rangle_{\varepsilon} Y\right]= & \left(\nabla_{X} A_{\alpha}\right)(Y)-\left(\nabla_{Y} A_{\alpha}\right)(X) \\
& +\sum_{\gamma}\left[A_{\gamma}(X) \omega_{\alpha}^{\gamma}(Y)-A_{\gamma}(Y) \omega_{\alpha}^{\gamma}(X)\right], \\
\left(d \omega_{\alpha}^{\beta}+\sum_{\gamma} \omega_{\gamma}^{\beta} \wedge \omega_{\alpha}^{\gamma}\right)(X, Y)= & \left\langle\left[A_{\alpha}, A_{\beta}\right](X), Y\right\rangle_{\varepsilon} .
\end{aligned}
$$

Demonstração. Para a equação (1.18), basta usar (1.14) em (1.1). Agora observe que 


$$
A_{\nabla_{X}^{\frac{1}{X}} e_{\alpha}} Y=A_{\sum_{\gamma} \omega_{\alpha}^{\gamma}(X) e_{\gamma}}(Y)=\sum_{\gamma} A_{\gamma}(Y) \omega_{\alpha}^{\gamma}(X)
$$

Usando a relação acima e (1.15) em (1.2), temos a equação (1.19).

Para (1.20), observe que pela equação (1.10) temos

$$
\begin{aligned}
\left(d \omega_{\alpha}^{\beta}+\sum_{\gamma} \omega_{\gamma}^{\beta} \wedge \omega_{\alpha}^{\gamma}\right)(X, Y) & =-\frac{1}{2} \sum_{k} \sum_{l} R_{k l \alpha}^{\perp \beta} \omega^{k} \wedge \omega^{l}(X, Y) \\
& =-\frac{1}{2}\left(\sum_{k} \sum_{l} R_{k l \alpha}^{\perp \beta} \omega^{k}(X) \omega^{l}(Y)-\sum_{k} \sum_{l} R_{k l \alpha}^{\perp \beta} \omega^{k}(Y) \omega^{l}(X)\right) .
\end{aligned}
$$

Note que

$$
\begin{aligned}
\sum_{k} \sum_{l} R_{k l \alpha}^{\perp \beta} \omega^{k}(X) \omega^{l}(Y) & =\sum_{k} \sum_{l}\left\langle R^{\perp}\left(e_{k}, e_{l}\right) e_{\alpha}, e_{\beta}\right\rangle_{\varepsilon} \omega^{k}(X) \omega^{l}(Y) \\
& =\left\langle R^{\perp}\left(\sum_{k} \omega^{k}(X) e_{k}, \sum_{l} \omega^{l}(Y) e_{l}\right) e_{\alpha}, e_{\beta}\right\rangle_{\varepsilon} \\
& =\left\langle R^{\perp}(X, Y) e_{\alpha}, e_{\beta}\right\rangle_{\varepsilon} .
\end{aligned}
$$

Pelas equações (1.3) e (1.16) temos

$$
\left\langle R^{\perp}(X, Y) e_{\alpha}, e_{\beta}\right\rangle_{\varepsilon}=-\left\langle\left[A_{\alpha}, A_{\beta}\right](X), Y\right\rangle_{\varepsilon},
$$

logo, substituindo na primeira equação segue que

$$
\begin{aligned}
\left(d \omega_{\alpha}^{\beta}+\sum_{\gamma} \omega_{\gamma}^{\beta} \wedge \omega_{\alpha}^{\gamma}\right)(X, Y) & =-\frac{1}{2}\left(-\left\langle\left[A_{\alpha}, A_{\beta}\right](X), Y\right\rangle_{\varepsilon}+\left\langle\left[A_{\alpha}, A_{\beta}\right](Y), X\right\rangle_{\varepsilon}\right) \\
& =\left\langle\left[A_{\alpha}, A_{\beta}\right](X), Y\right\rangle_{\varepsilon} .
\end{aligned}
$$

Note que $\frac{\partial}{\partial t}$ é paralelo. De fato, como $\frac{\partial}{\partial t}$ é unitário, temos que para todo $X \in \Gamma\left(T \mathbb{Q}^{m}(c) \times \mathbb{R}\right)$,

$$
\begin{aligned}
& \left\langle\frac{\partial}{\partial t}, \frac{\partial}{\partial t}\right\rangle_{\varepsilon}=1 \\
\Rightarrow \quad & X\left(\left\langle\frac{\partial}{\partial t}, \frac{\partial}{\partial t}\right\rangle_{\varepsilon}\right)=2\left\langle\bar{\nabla}_{X} \frac{\partial}{\partial t}, \frac{\partial}{\partial t}\right\rangle_{\varepsilon}=0 \\
\Rightarrow \quad & \left\langle\nabla_{\frac{\partial}{\partial t}}^{\mathbb{R}} \frac{\partial}{\partial t}, \frac{\partial}{\partial t}\right\rangle_{\varepsilon}=0 .
\end{aligned}
$$

Mas $\nabla_{\frac{\partial}{\partial t}}^{\mathbb{R}} \frac{\partial}{\partial t}$ é tangente a $\mathbb{R}$ em $\mathbb{Q}^{m}(c) \times \mathbb{R}, \log 0 \nabla_{\frac{\partial}{\partial t}}^{\mathbb{R}} \frac{\partial}{\partial t}=0$.

Proposição 1.3.5. Se $X \in \Gamma(T M)$ e $T$ e $\nu^{\gamma}$ são definidos como acima, temos

$$
\begin{gathered}
\nabla_{X} T-\sum_{\gamma} \nu^{\gamma} A_{\gamma} X=0 \\
\sum_{\gamma}\left\langle A_{\gamma} X, T\right\rangle_{\varepsilon} e_{\gamma}+\sum_{\gamma} d \nu^{\gamma}(X) e_{\gamma}+\sum_{\gamma} \nu^{\gamma}\left(\nabla_{X}^{\perp} e_{\gamma}\right)=0
\end{gathered}
$$


Demonstração. Como $\frac{\partial}{\partial t}$ é paralelo e $\frac{\partial}{\partial t}=T+\sum_{\gamma} \nu^{\gamma} e_{\gamma}$, temos

$$
\begin{aligned}
0 & =\bar{\nabla}_{X} \frac{\partial}{\partial t} \\
& =\bar{\nabla}_{X}\left(T+\sum_{\gamma} \nu^{\gamma} e_{\gamma}\right) \\
& =\bar{\nabla}_{X} T+\bar{\nabla}_{X} \sum_{\gamma} \nu^{\gamma} e_{\gamma}
\end{aligned}
$$

Mas $X \in \Gamma(T M)$, então

$$
\begin{aligned}
\bar{\nabla}_{X} T & =\nabla_{X} T+I I(X, T) \\
& =\nabla_{X} T+\sum_{\gamma}\left\langle I I(X, T), e_{\gamma}\right\rangle_{\varepsilon} e_{\gamma} \\
& =\nabla_{X} T+\sum_{\gamma}\left\langle A_{\gamma} X, T\right\rangle_{\varepsilon} e_{\gamma} .
\end{aligned}
$$

Além disso,

$$
\begin{aligned}
\bar{\nabla}_{X} \sum_{\gamma} \nu^{\gamma} e_{\gamma} & =\sum_{\gamma} d \nu^{\gamma}(X) e_{\gamma}+\sum_{\gamma} \nu^{\gamma} \bar{\nabla}_{X} e_{\gamma} \\
& =\sum_{\gamma} d \nu^{\gamma}(X) e_{\gamma}+\sum_{\gamma} \nu^{\gamma}\left(-A_{\gamma} X+\nabla_{X}^{\perp} e_{\gamma}\right) .
\end{aligned}
$$

Logo

$$
\begin{aligned}
0= & \nabla_{X} T+\sum_{\gamma}\left\langle A_{\gamma} X, T\right\rangle_{\varepsilon} e_{\gamma}+\sum_{\gamma} d \nu^{\gamma}(X) e_{\gamma}-\sum_{\gamma} \nu^{\gamma} A_{\gamma} X \\
& +\sum_{\gamma} \nu^{\gamma}\left(\nabla \frac{\perp}{X} e_{\gamma}\right) .
\end{aligned}
$$

Ambas as componentes tangencial e normal se anulam, portanto

$$
\nabla_{X} T-\sum_{\gamma} \nu^{\gamma} A_{\gamma} X=0
$$

e

$$
\sum_{\gamma}\left\langle A_{\gamma} X, T\right\rangle_{\varepsilon} e_{\gamma}+\sum_{\gamma} d \nu^{\gamma}(X) e_{\gamma}+\sum_{\gamma} \nu^{\gamma}\left(\nabla \frac{\perp}{X} e_{\gamma}\right)=0
$$




\section{Capítulo 2}

\section{Imersões Isométricas}

Note que no capítulo anterior nos restringimos apenas à subvariedades mergulhadas, que são, em particular, subconjuntos de $\mathbb{Q}_{\varepsilon}^{m} \times \mathbb{R}$. Na seção 2.1 deste capítulo, iniciaremos um estudo sobre as imersões isométricas nestes espaços. Dada uma imersão isométrica $f: M \longrightarrow \mathbb{Q}_{\varepsilon}^{m} \times \mathbb{R}$ de uma variedade $M$ de dimensão $n \leq m$, vamos transportar as relações encontradas na seção 1.3, que estariam definidas na imagem de $M$ por $f$, para $M$, evitando identificar abertos de $M$ com sua imagem por $f$. Desta forma, obteremos condições necessárias para que $M$ seja isometricamente imersa em $\mathbb{Q}_{\varepsilon}^{m} \times \mathbb{R}$.

Na seção 2.4, provaremos o Teorema Fundamental das Imersões em $\mathbb{Q}_{\varepsilon}^{m} \times \mathbb{R}$, mostrando que tais condições mencionadas acima não são apenas necessárias, mas, num certo sentido, suficientes para que, dada uma variedade Riemanniana simplesmente conexa $M$, exista uma imersão isométrica $f: M \longrightarrow \mathbb{Q}_{\varepsilon}^{m} \times \mathbb{R}$. Sua demonstração é motivada pelo estudo das subvariedades feito na seção 1.3, e utilizamos referenciais móveis e distribuições integráveis como em [Ten71].

\subsection{As Equações de Compatibilidade}

Seja $f: M \longrightarrow \mathbb{Q}_{\varepsilon}^{m} \times \mathbb{R}$ uma imersão isométrica de uma variedade $M$ de dimensão $n \leq m$ em $\mathbb{Q}_{\varepsilon}^{m} \times \mathbb{R}$. Denote por $\langle$,$\rangle e \langle,\rangle_{\varepsilon}$ as métricas Riemannianas, por $\nabla$ e $\bar{\nabla}$ as conexões Riemannianas e por $R$ e $\bar{R}$ os tensores curvatura de $M$ e $\mathbb{Q}_{\varepsilon}^{m} \times \mathbb{R}$ respecivamente. Como $f$ é uma isometria, temos que

$$
\langle u, v\rangle=\left\langle d f_{p}(u), d f_{p}(v)\right\rangle_{\varepsilon}
$$

para todo $p \in M$ e $u, v \in T_{p} M$. Além disso, por ser uma imersão, $f$ é localmente um mergulho. Logo, se $p \in M$ existe uma vizinhança $U$ de $p$ em $M$ tal que $f(U)$ é uma subvariedade de $\mathbb{Q}_{\varepsilon}^{m} \times \mathbb{R}$. Denotaremos por $\tilde{\nabla}$ a conexão Riemanniana e por $\tilde{R}$ o tensor curvatura de $f(U)$. Seja $\left\{\tilde{e}_{C}\right\}_{C=1}^{m+1}$ um referencial ortonormal local em $\mathbb{Q}_{\varepsilon}^{m} \times \mathbb{R}$ adaptado à $f(U)$, e seja $\left\{\tilde{\omega}_{C}\right\}_{C=1}^{m+1}$ sua base dual. Podemos supor que $\left\{\tilde{e}_{C}\right\}_{C=1}^{m+1}$ e $\left\{\tilde{\omega}_{C}\right\}_{C=1}^{m+1}$ estão definidos em todo $f(U)$. Faremos a mesma convenção de índices da seção 1.3. Sejam $\tilde{A}_{\alpha}$ os operadores forma associados aos campos normais $e_{\alpha}$ e tome as formas de conexão $\tilde{\omega}_{B}^{A}$ de $\mathbb{Q}_{\varepsilon}^{m} \times \mathbb{R}$ no referencial $\left\{e_{C}\right\}$. Portanto, como $f_{*}$ é um isomorfismo, para $X, Y, Z \in \Gamma(T U)$, as equações (1.18) - (1.22) são escritas em $f(U)$ da seguinte maneira:

$$
\begin{aligned}
\tilde{R}\left(f_{*} X, f_{*} Y\right) f_{*} Z= & \sum_{\gamma}\left[\left\langle\tilde{A}_{\gamma}\left(f_{*} X\right), f_{*} Z\right\rangle_{\varepsilon} \tilde{A}_{\gamma}\left(f_{*} Y\right)-\left\langle\tilde{A}_{\gamma}\left(f_{*} Y\right), f_{*} Z\right\rangle_{\varepsilon} \tilde{A}_{\gamma}\left(f_{*} X\right)\right] \\
& +\varepsilon\left[\left\langle f_{*} X, f_{*} Z\right\rangle_{\varepsilon} f_{*} Y-\left\langle f_{*} Y, f_{*} Z\right\rangle_{\varepsilon} f_{*} X\right. \\
& -\left\langle f_{*} Y, f_{*} T\right\rangle_{\varepsilon}\left\langle f_{*} X, f_{*} Z\right\rangle_{\varepsilon} f_{*} T-\left\langle f_{*} X, f_{*} T\right\rangle_{\varepsilon}\left\langle f_{*} Z, f_{*} T\right\rangle_{\varepsilon} f_{*} Y \\
& \left.+\left\langle f_{*} X, f_{*} T\right\rangle_{\varepsilon}\left\langle f_{*} Y, f_{*} Z\right\rangle_{\varepsilon} f_{*} T+\left\langle f_{*} Y, f_{*} T\right\rangle_{\varepsilon}\left\langle f_{*} Z, f_{*} T\right\rangle_{\varepsilon} f_{*} X\right],
\end{aligned}
$$




$$
\begin{aligned}
& \varepsilon \nu^{\alpha}\left[\left\langle f_{*} Y, f_{*} T\right\rangle_{\varepsilon} f_{*} X-\left\langle f_{*} X, f_{*} T\right\rangle_{\varepsilon} f_{*} Y\right]=\left(\tilde{\nabla}_{f_{*} X} \tilde{A}_{\alpha}\right)\left(f_{*} Y\right)-\left(\tilde{\nabla}_{f_{*} Y} \tilde{A}_{\alpha}\right)\left(f_{*} X\right) \\
&+\sum_{\gamma}\left[\tilde{A}_{\gamma}\left(f_{*} X\right) \tilde{\omega}_{\alpha}^{\gamma}\left(f_{*} Y\right)-\tilde{A}_{\gamma}\left(f_{*} Y\right) \tilde{\omega}_{\alpha}^{\gamma}\left(f_{*} X\right)\right], \\
&\left(d \tilde{\omega}_{\alpha}^{\beta}-\sum_{\gamma} \tilde{\omega}_{\alpha}^{\gamma} \wedge \tilde{\omega}_{\gamma}^{\beta}\right)\left(f_{*} X, f_{*} Y\right)=\left\langle\left[\tilde{A}_{\alpha}, \tilde{A}_{\beta}\right]\left(f_{*} X\right), f_{*} Y\right\rangle_{\varepsilon}, \\
& \tilde{\nabla}_{f_{*} X} f_{*} T-\sum_{\gamma} \tilde{\nu}^{\gamma} \tilde{A}_{\gamma}\left(f_{*} X\right)=0, \\
&\left\langle\tilde{A}_{\alpha}\left(f_{*} X\right), f_{*} T\right\rangle_{\varepsilon}+d \tilde{\nu}^{\alpha}\left(f_{*} X\right)+\sum_{\gamma} \tilde{\nu}^{\gamma} \tilde{\omega}_{\gamma}^{\alpha}\left(f_{*} X\right)=0,
\end{aligned}
$$

nas quais $f_{*} T$ é um campo de vetores em $f(U)$ e $\tilde{\nu}^{\gamma}$ são funções diferenciáveis em $f(U)$ tais que

$$
\frac{\partial}{\partial t}=f_{*} T+\sum_{\gamma} \tilde{\nu}^{\gamma} e_{\gamma}
$$

O próximo lema nos ajudará a escrever as equações acima no aberto $U$.

Lema 2.1.1. Como $f: U \longrightarrow f(U)$ é uma isometria, temos que para $X, Y, Z \in \Gamma(T U)$,

$$
f_{*} \nabla_{X} Y=\tilde{\nabla}_{f_{*} X} f_{*} Y \quad \text { e } \quad f_{*} R(X, Y) Z=\tilde{R}\left(f_{*} X, f_{*} Y\right) f_{*} Z .
$$

Demonstração. Defina a aplicação $f^{*} \tilde{\nabla}: \Gamma(T U) \times \Gamma(T U) \longrightarrow \Gamma(T U)$ por

$$
f^{*} \tilde{\nabla}_{X} Y=f_{*}^{-1} \tilde{\nabla}_{f_{*} X} f_{*} Y .
$$

Mostraremos que $f^{*} \tilde{\nabla}$ é a conexão Riemanniana em $M$. Sejam $X, Y, Z \in \Gamma(T U)$ e $g, h$ funções diferenciáveis em $U$. Então

$$
\begin{aligned}
\left(f^{*} \tilde{\nabla}\right)_{g X+h Y} Z & =f_{*}^{-1}\left(\tilde{\nabla}_{f_{*}(g X+h Y)} f_{*} Z\right) \\
& =f_{*}^{-1}\left(\tilde{\nabla}_{\left(\left(g \circ f^{-1}\right) f_{*} X+\left(h \circ f^{-1}\right) f_{*} Y\right)} f_{*} Z\right) \\
& =f_{*}^{-1}\left(\left(g \circ f^{-1}\right) \tilde{\nabla}_{f_{*} X} f_{*} Z+\left(h \circ f^{-1}\right) \tilde{\nabla}_{f_{*} Y} f_{*} Z\right) \\
& =\left(g \circ f^{-1} \circ f\right) f_{*}^{-1}\left(\tilde{\nabla}_{f_{*} X} f_{*} Z\right)+\left(h \circ f^{-1} \circ f\right) f_{*}^{-1}\left(\tilde{\nabla}_{f_{*} Y} f_{*} Z\right) \\
& =g\left(f^{*} \tilde{\nabla}\right)_{X} Z+h\left(f^{*} \tilde{\nabla}\right)_{Y} Z \\
\left(f^{*} \tilde{\nabla}\right)_{X}(Y+Z) & =f_{*}^{-1}\left(\tilde{\nabla}_{f_{*} X} f_{*}(Y+Z)\right) \\
& =f_{*}^{-1}\left(\tilde{\nabla}_{f_{*} X} f_{*} Y\right)+f_{*}^{-1}\left(\tilde{\nabla}_{f_{*} X} f_{*} Z\right) \\
& =\left(f^{*} \tilde{\nabla}_{X} Y+\left(f^{*} \tilde{\nabla}\right)_{X} Z\right. \\
& \\
\left(f^{*} \tilde{\nabla}\right)_{X} g Y & f_{*}^{-1}\left(\tilde{\nabla}_{f_{*} X} f_{*}(g Y)\right) \\
& =f_{*}^{-1}\left(\tilde{\nabla}_{f_{*} X}\left(g \circ f^{-1}\right) f_{*} Y\right) \\
& =f_{*}^{-1}\left(\left(f_{*} X\left(g \circ f^{-1}\right)\right) f_{*} Y+g \circ f^{-1} \tilde{\nabla}_{f_{*} X} f_{*} Y\right) \\
& =f_{*}^{-1}\left(\left(X(g) \circ f^{-1}\right) f_{*} Y\right)+f_{*}^{-1}\left(g \circ f^{-1} \tilde{\nabla}_{f_{*} X} f_{*} Y\right) \\
& \left.=\left(X(g) \circ f^{-1} \circ f\right) f_{*}^{-1} f_{*} Y\right)+\left(g \circ f^{-1} \circ f\right) f_{*}^{-1}\left(\tilde{\nabla}_{f_{*} X} f_{*} Y\right) \\
& =X(g) Y+g\left(f^{*} \tilde{\nabla}\right)_{X} Y
\end{aligned}
$$




$$
\begin{aligned}
X\langle Y, Z\rangle & =X\left(\left\langle f_{*} Y, f_{*} Z\right\rangle_{\varepsilon} \circ f\right) \\
& =\left[\left(f_{*} X\right)\left(\left\langle f_{*} Y, f_{*} Z\right\rangle_{\varepsilon}\right)\right] \circ f \\
& =\left\langle\tilde{\nabla}_{f_{*} X} f_{*} Y, f_{*} Z\right\rangle_{\varepsilon} \circ f+\left\langle f_{*} Y, \tilde{\nabla}_{f_{*} X} f_{*} Z\right\rangle_{\varepsilon} \circ f \\
& =\left\langle f_{*}^{-1}\left(\tilde{\nabla}_{f_{*} X} f_{*} Y\right), Z\right\rangle+\left\langle Y, f_{*}^{-1}\left(\tilde{\nabla}_{f_{*} X} f_{*} Z\right)\right\rangle \\
& =\left\langle\left(f^{*} \tilde{\nabla}\right)_{X} Y, Z\right\rangle+\left\langle Y,\left(f^{*} \tilde{\nabla}\right)_{X} Z\right\rangle, \\
& \\
\left(f^{*} \tilde{\nabla}\right)_{X} Y-\left(f^{*} \tilde{\nabla}\right)_{Y} X & =f_{*}^{-1}\left(\tilde{\nabla}_{f_{*} X} f_{*} Y\right)-f_{*}^{-1}\left(\tilde{\nabla}_{f_{*} Y} f_{*} X\right) \\
& =f_{*}^{-1}\left(\tilde{\nabla}_{f_{*} X} f_{*} Y-\tilde{\nabla}_{f_{*} Y} f_{*} X\right) \\
& =f_{*}^{-1}\left[f_{*} X, f_{*} Y\right] \\
& =\left[f_{*}^{-1} f_{*} X, f_{*}^{-1} f_{*} Y\right] \\
& =[X, Y] .
\end{aligned}
$$

Portanto $f^{*} \tilde{\nabla}$ é conexão Riemanniana em $U$. Pela unicidade da conexão, segue que

$$
\nabla=f^{*} \tilde{\nabla} \Rightarrow \nabla_{X} Y=f_{*}^{-1} \tilde{\nabla}_{f_{*} X} f_{*} Y \Rightarrow f_{*} \nabla_{X} Y=\tilde{\nabla}_{f_{*} X} f_{*} Y,
$$

como queríamos. Para a segunda equação do lema, observe que, usando o que fizemos até agora, temos

$$
\begin{aligned}
f_{*} R(X, Y) Z & =f_{*}\left(\nabla_{Y} \nabla_{X} Z-\nabla_{X} \nabla_{Y} Z+\nabla_{[X, Y]} Z\right) \\
& =f_{*}\left(\nabla_{Y} \nabla_{X} Z\right)-f_{*}\left(\nabla_{X} \nabla_{Y} Z\right)+f_{*}\left(\nabla_{[X, Y]} Z\right) \\
& =\tilde{\nabla}_{f_{*} Y} f_{*} \nabla_{X} Z-\tilde{\nabla}_{f_{*} X} f_{*} \nabla_{Y} Z+\nabla_{f_{*}[X, Y]} f_{*} Z \\
& =\tilde{\nabla}_{f_{*} Y} \tilde{\nabla}_{f_{*} X} f_{*} Z-\tilde{\nabla}_{f_{*} X} \tilde{\nabla}_{f_{*} Y} f_{*} Z+\nabla_{\left[f_{*} X, f_{*} Y\right]} f_{*} Z \\
& =\tilde{R}\left(f_{*} X, f_{*} Y\right) f_{*} Z .
\end{aligned}
$$

Defina em $U$ os tensores simétricos $A_{\gamma}: T U \longrightarrow T U$ por

$$
A_{\gamma}=f_{*}^{-1} \tilde{A}_{\gamma} f_{*}
$$

as 1-formas $\omega_{\gamma}^{\alpha}$ por

$$
\omega_{\gamma}^{\alpha}(X)=\left(f^{*} \tilde{\omega}_{\gamma}^{\alpha}\right)(X)=\tilde{\omega}_{\gamma}^{\alpha}\left(f_{*} X\right)
$$

e funções $\nu^{\gamma}$ por

$$
\tilde{\nu}^{\gamma}=\nu^{\gamma} \circ f^{-1}
$$

Desta forma, usando o lema 2.1.1 e o fato de $f_{*}$ ser um isomorfismo linear e uma isometria, as equações (1.18) - (1.22) podem agora ser reescritas em $U$ como

$$
\begin{aligned}
R(X, Y) Z= & \sum_{\gamma}\left[\left\langle A_{\gamma}(X), Z\right\rangle A_{\gamma}(Y)-\left\langle A_{\gamma}(Y), Z\right\rangle A_{\gamma}(X)\right] \\
& +\varepsilon[\langle X, Z\rangle Y-\langle Y, Z\rangle X \\
& -\langle Y, T\rangle\langle X, Z\rangle T-\langle X, T\rangle\langle Z, T\rangle Y \\
& +\langle X, T\rangle\langle Y, Z\rangle T+\langle Y, T\rangle\langle Z, T\rangle X]
\end{aligned}
$$




$$
\begin{aligned}
& \varepsilon \nu^{\alpha}[\langle Y, T\rangle X-\langle X, T\rangle Y]=\left(\nabla_{X} A_{\alpha}\right)(Y)-\left(\nabla_{Y} A_{\alpha}\right)(X) \\
&+\sum_{\gamma}\left[A_{\gamma}(X) \omega_{\alpha}^{\gamma}(Y)-A_{\gamma}(Y) \omega_{\alpha}^{\gamma}(X)\right], \\
&\left(d \omega_{\alpha}^{\beta}-\sum_{\gamma} \omega_{\alpha}^{\gamma} \wedge \omega_{\gamma}^{\beta}\right)(X, Y)=\left\langle\left[A_{\alpha}, A_{\beta}\right] X, Y\right\rangle, \\
& \nabla_{X} T-\sum_{\gamma} \nu^{\gamma} A_{\gamma}(X)=0, \\
&\left\langle A_{\alpha}(X), T\right\rangle+d \nu^{\alpha}(X)+\sum_{\gamma} \nu^{\gamma} \omega_{\gamma}^{\alpha}(X)=0 .
\end{aligned}
$$

Portanto estas relações locais são condições necessárias para que a variedade $M$ seja isometricamente imersa em $\mathbb{Q}_{\varepsilon}^{m} \times \mathbb{R}$. Isso nos motiva a seguinte definição.

Definição 2.1.2. Seja $M$ uma variedade Riemanniana de dimensão $n$, com métrica $\langle$,$\rangle , conexão$ Riemanniana $\nabla$ e tensor curvatura $R$. Sejam $A_{\alpha}: T M \longrightarrow T M$ tensores simétricos em $M$ e $\omega_{\alpha}^{\beta}$ 1-formas anti-simétricas em $M$. Sejam também $T$ um campo de vetores em $M$ e $\nu^{\alpha}$ funções diferenciáveis em $M$ tais que $\|T\|^{2}+\sum_{\alpha}\left(\nu^{\alpha}\right)^{2}=1$. Dizemos que $\left(\langle\rangle,, A_{\alpha}, \omega_{\alpha}^{\beta}, T, \nu^{\alpha}\right)$ satisfazem as equações de compatibilidade para $\mathbb{Q}_{\varepsilon}^{m} \times \mathbb{R}$ se, para todo $X, Y, Z \in \Gamma(T M)$, valem as seguintes relações:

$$
\begin{gathered}
R(X, Y) Z=\sum_{\gamma}\left[\left\langle A_{\gamma}(X), Z\right\rangle A_{\gamma}(Y)-\left\langle A_{\gamma}(Y), Z\right\rangle A_{\gamma}(X)\right] \\
+\varepsilon[\langle X, Z\rangle Y-\langle Y, Z\rangle X \\
-\langle Y, T\rangle\langle X, Z\rangle T-\langle X, T\rangle\langle Z, T\rangle Y \\
+\langle X, T\rangle\langle Y, Z\rangle T+\langle Y, T\rangle\langle Z, T\rangle X], \\
\varepsilon \nu^{\alpha}[\langle Y, T\rangle X-\langle X, T\rangle Y]=\quad\left(\nabla_{X} A_{\alpha}\right)(Y)-\left(\nabla_{Y} A_{\alpha}\right)(X) \\
\quad+\sum_{\gamma}\left[A_{\gamma}(X) \omega_{\alpha}^{\gamma}(Y)-A_{\gamma}(Y) \omega_{\alpha}^{\gamma}(X)\right], \\
\left(d \omega_{\alpha}^{\beta}-\sum_{\gamma} \omega_{\alpha}^{\gamma} \wedge \omega_{\gamma}^{\beta}\right)(X, Y)=\left\langle\left[A_{\alpha}, A_{\beta}\right](X), Y\right\rangle, \\
\nabla_{X} T-\sum_{\gamma} \nu^{\gamma} A_{\gamma}(X)=0, \\
\left\langle A_{\alpha}(X), T\right\rangle+d \nu^{\alpha}(X)+\sum_{\gamma} \nu^{\gamma} \omega_{\gamma}^{\alpha}(X)=0 .
\end{gathered}
$$

Chamaremos uma quíntupla $\left(\langle\rangle,, A_{\alpha}, \omega_{\alpha}^{\beta}, T, \nu^{\alpha}\right)$ satisfazendo as equações de compatibilidade para $\mathbb{Q}_{\varepsilon}^{m} \times \mathbb{R}$ de dados compativeis para $M$.

Se existe uma imersão isométrica $f: M \rightarrow \mathbb{Q}_{\varepsilon}^{n} \times \mathbb{R}$, então $f$ determina os dados compatíveis para $M$, como foi visto acima. No caso em que $M$ tem dimensão $n$, temos apenas uma direção 
normal, e como as 1-formas $\omega_{n+1}^{n+1}=0$, pois são anti-simétricas, então os dados compatíveis podem ser descritos apenas pela quádrupla $(\langle\rangle, A, T,, \nu)$.

Mostraremos na próxima seção que as equações de compatibilidade não são apenas condições necessárias para $M$ ser localmente isometricamente imersa em $\mathbb{Q}_{\varepsilon}^{m} \times \mathbb{R}$, mas também suficientes. Além disso, a imersão será única a menos de uma isometria global de $\mathbb{Q}_{\varepsilon}^{m} \times \mathbb{R}$ que preserva as orientações de $\mathbb{Q}_{\varepsilon}^{m}$ e $\mathbb{R}$, e se $M$ for simplesmente conexa, a imersão é global.

\section{$2.2 \quad$ Imersões isométricas em $\mathbb{S}^{m} \times \mathbb{R}$ e $\mathbb{H}^{m} \times \mathbb{R}$}

Seja $M$ uma variedade Riemanniana simplesmente conexa de dimensão $n$, com métrica $\langle$,$\rangle ,$ conexão $\nabla$ e tensor curvatura $R$. Para $n+1 \leq \alpha, \beta \leq m+1$, sejam $A_{\alpha}: T M \rightarrow T M$ tensores simétricos em $M, \omega_{\alpha}^{\beta}$ 1-formas anti-simétricas em $M$. Sejam também $T$ um campo de vetores em $M$ e $\nu^{\alpha}$ funções diferenciáveis em $M$ tais que $\|T\|^{2}+\sum_{\alpha}\left(\nu^{\alpha}\right)^{2}=1$.

Considere um referencial ortonormal $\left\{e_{i}\right\}_{i=1}^{n}$ em $M$. Seja $\left\{\omega^{i}\right\}_{i=1}^{n}$ a base dual de $\left\{e_{i}\right\}_{i=1}^{n}$ e $\left\{\omega_{j}^{i}\right\}_{i, j=1}^{n}$ as formas de conexão de $M$ no referencial $\left\{e_{i}\right\}_{i=1}^{n}$.

Definimos a 1-forma $\eta$ em $M$ por

$$
\eta(X)=\langle T, X\rangle
$$

e escrevemos

$$
T^{0}=0, \quad T^{k}=\left\langle T, e_{k}\right\rangle, \quad T^{\alpha}=\nu^{\alpha} .
$$

Como $\eta\left(e_{k}\right)=\left\langle T, e_{k}\right\rangle=T^{k}$, no referencial $\left\{e_{i}\right\}_{i=1}^{n}$ podemos escrever $\eta=\sum_{k} T^{k} \omega^{k}$. Definimos também as 1-formas em $M$

$$
\begin{aligned}
& \omega_{j}^{\alpha}=\sum_{k}\left\langle A_{\alpha}\left(e_{k}\right), e_{j}\right\rangle \omega^{k}, \quad \omega_{\alpha}^{j}=-\omega_{j}^{\alpha} . \\
& \omega_{j}^{0}=\varepsilon\left(T^{j} \eta-\omega^{j}\right), \quad \omega_{0}^{j}=-\varepsilon \omega_{j}^{0}, \\
& \omega_{\alpha}^{0}=\varepsilon \nu^{\alpha} \eta, \quad \omega_{0}^{\alpha}=-\varepsilon \omega_{\alpha}^{0}, \\
& \omega_{0}^{0}=0 .
\end{aligned}
$$

A partir da definição de $\omega_{j}^{\alpha}$, temos a seguinte proposição, cuja demonstração é análoga as demonstrações de (1.7) e (1.9) em (1.2.3).

Proposição 2.2.1. Valem as seguintes equações em $M$ :

$$
\begin{aligned}
& \sum_{p} \omega_{p}^{\alpha} \wedge \omega^{p}=0 \\
& d \omega_{j}^{\alpha}+\sum_{p} \omega_{p}^{\alpha} \wedge \omega_{j}^{p}=\frac{1}{2} \sum_{k, l}\left\langle\left(\nabla_{e_{k}} A_{\alpha}\right)\left(e_{l}\right)-\left(\nabla_{e_{l}} A_{\alpha}\right)\left(e_{k}\right), e_{j}\right\rangle .
\end{aligned}
$$

Vamos assumir que $\left(\langle\rangle,, A_{\alpha}, \omega_{\alpha}^{\beta}, T, \nu^{\alpha}\right)$ satisfaz as equações de compatibilidade para $\mathbb{Q}_{\varepsilon}^{m} \times \mathbb{R}$. Provaremos agora algumas consequências destas equações.

Lema 2.2.2. Temos que

$$
d \eta=0
$$

Demonstração. Para $X, Y \in \Gamma(T M)$, temos

$$
\begin{aligned}
d \eta(X, Y) & =X \eta(Y)-Y \eta(X)=\eta([X, Y]) \\
& =X\langle T, Y\rangle-Y\langle T, X\rangle-\langle T,[X, Y]\rangle \\
& =\left\langle\nabla_{X} T, Y\right\rangle+\left\langle T, \nabla_{X} Y\right\rangle-\left\langle\nabla_{Y} T, X\right\rangle-\left\langle T, \nabla_{Y} X\right\rangle-\left\langle T, \nabla_{X} Y-\nabla_{Y} X\right\rangle \\
& =\left\langle\nabla_{X} T, Y\right\rangle-\left\langle\nabla_{Y} T, X\right\rangle
\end{aligned}
$$


Como $\nabla_{X} T=\sum_{\gamma} \nu^{\gamma} A_{\gamma}(X)$, segue que

$$
\begin{aligned}
d \eta(X, Y) & =\left\langle\sum_{\gamma} \nu^{\gamma} A_{\gamma}(X), Y\right\rangle-\left\langle\sum_{\gamma} \nu^{\gamma} A_{\gamma}(Y), X\right\rangle \\
& =\sum_{\gamma} \nu^{\gamma}\left[\left\langle A_{\gamma}(X), Y\right\rangle-\left\langle A_{\gamma}(Y), X\right\rangle\right] \\
& =0,
\end{aligned}
$$

pois $A_{\gamma}$ é simétrico.

Proposição 2.2.3. Valem as seguintes fórmulas

$$
\begin{aligned}
& d \omega_{j}^{i}+\sum_{p} \omega_{p}^{i} \wedge \omega_{j}^{p}+\sum_{\gamma} \omega_{\gamma}^{i} \wedge \omega_{j}^{\gamma}=\varepsilon\left(\omega^{i} \wedge \omega^{j}+T^{i} \omega^{j} \wedge \eta-T^{j} \omega^{i} \wedge \eta\right) \\
& d \omega_{j}^{\alpha}+\sum_{p} \omega_{p}^{\alpha} \wedge \omega_{j}^{p}+\sum_{\gamma} \omega_{\gamma}^{\alpha} \wedge \omega_{j}^{\gamma}=\varepsilon \nu^{\alpha} \omega^{j} \wedge \eta \\
& d \omega_{\beta}^{\alpha}+\sum_{p} \omega_{p}^{\alpha} \wedge \omega_{\beta}^{p}+\sum_{\gamma} \omega_{\gamma}^{\alpha} \wedge \omega_{\beta}^{\gamma}=0 \\
& d T^{i}+\sum_{p} T^{p} \omega_{p}^{i}+\sum_{\gamma} \nu^{\gamma} \omega_{\gamma}^{i}=0 \\
& d \nu^{\alpha}+\sum_{p} T^{p} \omega_{p}^{\alpha}+\sum_{\gamma} \nu^{\gamma} \omega_{\gamma}^{\alpha}=0 .
\end{aligned}
$$

Demonstração. Pela equação de compatibilidade (2.1) temos que

$$
\begin{aligned}
R_{k l j}^{i}=\left\langle R\left(e_{k}, e_{l}\right) e_{j}, e_{i}\right\rangle= & \sum_{\gamma}\left[\left\langle A_{\gamma}\left(e_{k}\right), e_{j}\right\rangle\left\langle A_{\gamma}\left(e_{l}\right), e_{i}\right\rangle-\left\langle A_{\gamma}\left(e_{l}\right), e_{j}\right\rangle\left\langle A_{\gamma}\left(e_{k}\right), e_{i}\right\rangle\right] \\
& +\varepsilon\left(\left\langle e_{k}, e_{j}\right\rangle\left\langle e_{l}, e_{i}\right\rangle+\left\langle e_{k}, T\right\rangle\left\langle e_{l}, e_{j}\right\rangle\left\langle T, e_{i}\right\rangle+\left\langle e_{l}, T\right\rangle\left\langle e_{j}, T\right\rangle\left\langle e_{k}, e_{i}\right\rangle\right. \\
& \left.-\left\langle e_{l}, e_{j}\right\rangle\left\langle e_{k}, e_{i}\right\rangle-\left\langle e_{l}, T\right\rangle\left\langle e_{k}, e_{j}\right\rangle\left\langle T, e_{i}\right\rangle-\left\langle e_{k}, T\right\rangle\left\langle e_{j}, T\right\rangle\left\langle e_{l}, e_{i}\right\rangle\right) \\
= & \sum_{\gamma}\left[\omega_{j}^{\gamma}\left(e_{k}\right) \omega_{i}^{\gamma}\left(e_{l}\right)-\omega_{j}^{\gamma}\left(e_{l}\right) \omega_{i}^{\gamma}\left(e_{k}\right)\right] \\
& +\varepsilon\left(\delta_{k j} \delta_{l i}+T^{k} T^{i} \delta_{l j}+T^{l} T^{j} \delta_{k i}-\delta_{l j} \delta_{k i}-T^{l} T^{i} \delta_{k j}-T^{k} T^{j} \delta_{l i}\right) .
\end{aligned}
$$

Observe que:

$$
\begin{aligned}
& \begin{aligned}
\omega_{j}^{\gamma}\left(e_{k}\right) \omega_{i}^{\gamma}\left(e_{l}\right)-\omega_{j}^{\gamma}\left(e_{l}\right) \omega_{i}^{\gamma}\left(e_{k}\right) & =\omega_{j}^{\gamma} \wedge \omega_{i}^{\gamma}\left(e_{k}, e_{l}\right) \\
& =\omega_{\gamma}^{i} \wedge \omega_{j}^{\gamma}\left(e_{k}, e_{l}\right),
\end{aligned} \\
& \delta_{k j} \delta_{l i}-\delta_{l j} \delta_{k i}= \omega^{j}\left(e_{k}\right) \omega^{i}\left(e_{l}\right)-\omega^{j}\left(e_{l}\right) \omega^{i}\left(e_{k}\right) \\
&=-\omega^{i} \wedge \omega^{j}\left(e_{k}, e_{l}\right), \\
& T^{k} T^{i} \delta_{l j}-T^{l} T^{i} \delta_{k j}=T^{i}\left(\eta\left(e_{k}\right) \omega^{j}\left(e_{l}\right)-\eta\left(e_{l}\right) \omega^{j}\left(e_{k}\right)\right) \\
&=-T^{i} \omega^{j} \wedge \eta\left(e_{k}, e_{l}\right),
\end{aligned}
$$

e

$$
\begin{aligned}
T^{l} T^{j} \delta_{k i}-T^{k} T^{j} \delta_{l i} & =T^{j}\left(\eta\left(e_{l}\right) \omega^{i}\left(e_{k}\right)-\eta\left(e_{k}\right) \omega^{i}\left(e_{l}\right)\right) \\
& =T^{j} \omega^{i} \wedge \eta\left(e_{k}, e_{l}\right)
\end{aligned}
$$


Então, pelas relações acima, temos

$$
R_{k l j}^{i}=\sum_{\gamma} \omega_{j}^{\gamma} \wedge \omega_{i}^{\gamma}\left(e_{k}, e_{l}\right)-\varepsilon\left(\omega^{i} \wedge \omega^{j}+T^{i} \omega^{j} \wedge \eta-T^{j} \omega^{i} \wedge \eta\right)\left(e_{k}, e_{l}\right) .
$$

Por (1.8), temos que

$$
\begin{aligned}
d \omega_{j}^{i}\left(e_{k}, e_{l}\right)+\sum_{p} \omega_{p}^{i} \wedge \omega_{j}^{p}\left(e_{k}, e_{l}\right) & =-\frac{1}{2} \sum_{r, s} R_{r s j}^{i} \omega^{r} \wedge \omega^{s}\left(e_{k}, e_{l}\right) \\
& =-\frac{1}{2} \sum_{r, s} R_{r s j}^{i}\left(\omega^{r}\left(e_{k}\right) \omega^{s}\left(e_{l}\right)-\omega^{r}\left(e_{l}\right) \omega^{s}\left(e_{k}\right)\right) \\
& =-\frac{1}{2} \sum_{r, s} R_{r s j}^{i}\left(\delta_{r k} \delta_{s l}-\delta_{r l} \delta_{s k}\right) \\
& =-\frac{1}{2}\left(R_{k l j}^{i}-R_{l k j}^{i}\right) \\
& =-R_{k l j}^{i} .
\end{aligned}
$$

Logo, pela equação acima temos que

$$
d \omega_{j}^{i}+\sum_{p} \omega_{p}^{i} \wedge \omega_{j}^{p}=-\sum_{\gamma} \omega_{\gamma}^{i} \wedge \omega_{j}^{\gamma}+\varepsilon\left(\omega^{i} \wedge \omega^{j}+T^{i} \omega^{j} \wedge \eta-T^{j} \omega^{i} \wedge \eta\right)
$$

e segue a equação (2.8).

Observe que

$$
\begin{aligned}
\varepsilon \nu^{\alpha}\left(\left\langle e_{l}, T\right\rangle\left\langle e_{k}, e_{j}\right\rangle-\left\langle e_{k}, T\right\rangle\left\langle e_{l}, e_{j}\right\rangle\right) & =\varepsilon \nu^{\alpha}\left(\eta\left(e_{l}\right) \omega^{j}\left(e_{k}\right)-\eta\left(e_{k}\right) \omega^{j}\left(e_{l}\right)\right) \\
& =\varepsilon \nu^{\alpha} \omega^{j} \wedge \eta\left(e_{k}, e_{l}\right)
\end{aligned}
$$

e

$$
\begin{aligned}
\left\langle\sum_{\gamma}\left[A_{\gamma}\left(e_{k}\right) \omega_{\alpha}^{\gamma}\left(e_{l}\right)-A_{\gamma}\left(e_{l}\right) \omega_{\alpha}^{\gamma}\left(e_{k}\right)\right], e_{j}\right\rangle & =\sum_{\gamma}\left[\omega_{\alpha}^{\gamma}\left(e_{l}\right)\left\langle A_{\gamma}\left(e_{k}\right), e_{j}\right\rangle-\omega_{\alpha}^{\gamma}\left(e_{k}\right)\left\langle A_{\gamma}\left(e_{l}\right), e_{j}\right\rangle\right] \\
& =\sum_{\gamma}\left[\omega_{\alpha}^{\gamma}\left(e_{l}\right) \omega_{j}^{\gamma}\left(e_{k}\right)-\omega_{\alpha}^{\gamma}\left(e_{k}\right) \omega_{j}^{\gamma}\left(e_{l}\right)\right] \\
& =\sum_{\gamma} \omega_{\gamma}^{\alpha} \wedge \omega_{j}^{\gamma}\left(e_{k}, e_{l}\right) .
\end{aligned}
$$

Pela equação de compatibilidade (2.2) temos que

$$
\varepsilon \nu^{\alpha} \omega^{j} \wedge \eta\left(e_{k}, e_{l}\right)=\left\langle\left(\nabla_{e_{k}} A_{\alpha}\right)\left(e_{l}\right)-\left(\nabla_{e_{l}} A_{\alpha}\right)\left(e_{k}\right), e_{j}\right\rangle+\sum_{\gamma} \omega_{\gamma}^{\alpha} \wedge \omega_{j}^{\gamma}\left(e_{k}, e_{l}\right) .
$$

Por (2.7), temos que

$$
\begin{aligned}
d \omega_{j}^{\alpha}\left(e_{k}, e_{l}\right)+\sum_{p} \omega_{p}^{\alpha} \wedge \omega_{j}^{p}\left(e_{k}, e_{l}\right)= & \frac{1}{2} \sum_{r, s}\left\langle\left(\nabla_{e_{k}} A_{\alpha}\right)\left(e_{l}\right)-\left(\nabla_{e_{l}} A_{\alpha}\right)\left(e_{k}\right), e_{j}\right\rangle \omega^{k} \wedge \omega^{l}\left(e_{k}, e_{l}\right) \\
= & \frac{1}{2} \sum_{r, s}\left\langle\left(\nabla_{e_{k}} A_{\alpha}\right)\left(e_{l}\right)-\left(\nabla_{e_{l}} A_{\alpha}\right)\left(e_{k}\right), e_{j}\right\rangle\left(\omega^{r}\left(e_{k}\right) \omega^{s}\left(e_{l}\right)\right. \\
& \left.-\omega^{r}\left(e_{l}\right) \omega^{s}\left(e_{k}\right)\right) \\
= & \frac{1}{2} \sum_{r, s}\left\langle\left(\nabla_{e_{k}} A_{\alpha}\right)\left(e_{l}\right)-\left(\nabla_{e_{l}} A_{\alpha}\right)\left(e_{k}\right), e_{j}\right\rangle\left(\delta_{r k} \delta_{s l}-\delta_{r l} \delta_{s k}\right) \\
= & \left\langle\left(\nabla_{e_{k}} A_{\alpha}\right)\left(e_{l}\right)-\left(\nabla_{e_{l}} A_{\alpha}\right)\left(e_{k}\right), e_{j}\right\rangle .
\end{aligned}
$$


Logo, pela equação acima temos

$$
d \omega_{j}^{\alpha}+\sum_{p} \omega_{p}^{\alpha} \wedge \omega_{j}^{p}=\varepsilon \nu^{\alpha} \omega^{j} \wedge \eta-\sum_{\gamma} \omega_{\gamma}^{\alpha} \wedge \omega_{j}^{\gamma},
$$

e segue a equação (2.9).

Pela equação de compatibilidade (2.3), temos

$$
\begin{aligned}
\left(d \omega_{\alpha}^{\beta}+\sum_{\gamma} \omega_{\gamma}^{\beta} \wedge \omega_{\alpha}^{\gamma}\right)(X, Y) & =\left\langle\left[A_{\alpha}, A_{\beta}\right](X), Y\right\rangle \\
& =\left\langle A_{\alpha}\left(A_{\beta}(X)\right)-A_{\beta}\left(A_{\alpha}(X)\right), Y\right\rangle \\
& =\left\langle A_{\alpha}\left(A_{\beta}(X)\right), Y\right\rangle-\left\langle A_{\beta}\left(A_{\alpha}(X)\right), Y\right\rangle \\
& =\left\langle A_{\beta}(X), A_{\alpha}(Y)\right\rangle-\left\langle A_{\alpha}(X), A_{\beta}(Y)\right\rangle \\
& =\left\langle\sum_{p}\left\langle A_{\beta}(X), e_{p}\right\rangle e_{p}, A_{\alpha}(Y)\right\rangle-\left\langle\sum_{p}\left\langle A_{\alpha}(X), e_{p}\right\rangle e_{p}, A_{\beta}(Y)\right\rangle \\
& =\sum_{p}\left\langle A_{\beta}(X), e_{p}\right\rangle\left\langle e_{p}, A_{\alpha}(Y)\right\rangle-\sum_{p}\left\langle A_{\alpha}(X), e_{p}\right\rangle\left\langle e_{p}, A_{\beta}(Y)\right\rangle \\
& =\sum_{p}\left[\omega_{p}^{\beta}(X) \omega_{p}^{\alpha}(Y)-\omega_{p}^{\beta}(Y) \omega_{p}^{\alpha}(X)\right] \\
& =-\sum_{p} \omega_{p}^{\beta} \wedge \omega_{\alpha}^{p}(X, Y),
\end{aligned}
$$

e segue a equação (2.10).

Pela equação de compatibilidade (2.4) temos

$$
\begin{aligned}
0 & =\left\langle\nabla_{X} T, e_{i}\right\rangle-\left\langle\sum_{\gamma} \nu^{\gamma} A_{\gamma}(X), e_{i}\right\rangle \\
& =X\left\langle T, e_{i}\right\rangle-\left\langle T, \nabla_{X} e_{i}\right\rangle-\sum_{\gamma} \nu^{\gamma}\left\langle A_{\gamma}(X), e_{i}\right\rangle \\
& =X\left(T^{i}\right)-\left\langle T, \sum_{p}\left\langle\nabla_{X} e_{i}, e_{p}\right\rangle e_{p}\right\rangle-\sum_{\gamma} \nu^{\gamma} \omega_{i}^{\gamma}(X) \\
& =d T^{i}(X)+\sum_{p} T^{p} \omega_{p}^{i}(X)+\sum_{\gamma} \nu^{\gamma} \omega_{\gamma}^{i}(X)
\end{aligned}
$$

e segue a equação (2.11).

Como

$$
\begin{aligned}
\left\langle A_{\alpha}(X), T\right\rangle & =\left\langle\sum_{p}\left\langle A_{\alpha}(X), e_{p}\right\rangle e_{p}, T\right\rangle \\
& =\sum_{p}\left\langle A_{\alpha}(X), e_{p}\right\rangle\left\langle e_{p}, T\right\rangle \\
& =\sum_{p} \omega_{p}^{\alpha}(X) T^{p},
\end{aligned}
$$

pela equação de compatibilidade (2.5) segue a equação (2.12).

Lema 2.2.4. Temos

$$
d T^{B}=\sum_{C} T^{C} \omega_{B}^{C}
$$

Demonstração. Dividiremos em três casos. 
Caso $B=0$ : Temos que $d T^{0}=0$. Por outro lado,

$$
\begin{aligned}
\sum_{C} T^{C} \omega_{0}^{C} & =\sum_{j} T^{j} \omega_{0}^{j}+\sum_{\alpha} T^{\alpha} \omega_{0}^{\alpha} \\
& =-\sum_{j}\left(T^{j} \eta-\omega^{j}\right)-\sum_{\alpha} T^{\alpha} \nu^{\alpha} \eta \\
& =-\left(\sum_{j}\left(T^{j}\right)^{2}+\sum_{\alpha}\left(\nu^{\alpha}\right)^{2}\right) \eta+\sum_{j} T^{j} \omega^{j} .
\end{aligned}
$$

Como $\|T\|^{2}+\sum_{\alpha}\left(\nu^{\alpha}\right)^{2}=1$, temos

$$
\sum_{C} T^{C} \omega_{0}^{C}=-\eta+\sum_{j} T^{j} \omega^{j}=0
$$

Caso $1 \leq B \leq n$ : Pela equação de compatibilidade (2.4), temos que

$$
\left\langle\nabla_{e_{k}} T, e_{B}\right\rangle=\left\langle\sum_{\gamma} \nu^{\gamma} A_{\gamma}\left(e_{k}\right), e_{B}\right\rangle
$$

Como

$$
\begin{aligned}
\left\langle\nabla_{e_{k}} T, e_{B}\right\rangle & =e_{k}\left\langle T, e_{B}\right\rangle-\left\langle T, \nabla_{e_{k}} e_{B}\right\rangle \\
& =e_{k}\left(T^{B}\right)-\left\langle T, \sum_{j} \omega_{B}^{j}\left(e_{k}\right) e_{j}\right\rangle \\
& =d T^{B}\left(e_{k}\right)-\sum_{j} T^{j} \omega_{B}^{j}\left(e_{k}\right)
\end{aligned}
$$

e

$$
\left\langle\sum_{\gamma} \nu^{\gamma} A_{\gamma}\left(e_{k}\right), e_{B}\right\rangle=\sum_{\gamma} \nu^{\gamma}\left\langle A_{\gamma}\left(e_{k}\right), e_{B}\right\rangle=\sum_{\gamma} T^{\gamma} \omega_{B}^{\gamma}\left(e_{k}\right)
$$

segue que

$$
d T^{B}\left(e_{k}\right)=\sum_{j} T^{j} \omega_{B}^{j}\left(e_{k}\right)+\sum_{\gamma} T^{\gamma} \omega_{B}^{\gamma}\left(e_{k}\right)
$$

Caso $n+1 \leq B \leq m+1$ : Pela equação de compatibilidade (2.5) temos que

$$
\left\langle A_{B}\left(e_{k}\right), T\right\rangle+d \nu^{B}\left(e_{k}\right)+\sum_{\gamma} \nu^{\gamma} \omega_{\gamma}^{B}\left(e_{k}\right)=0
$$

Como

$$
\begin{aligned}
\left\langle A_{B}\left(e_{k}\right), T\right\rangle & =\left\langle A_{B}\left(e_{k}\right), \sum_{j}\left\langle T, e_{j}\right\rangle e_{j}\right\rangle \\
& =\sum_{j} T^{j}\left\langle A_{B}\left(e_{k}\right), e_{j}\right\rangle \\
& =-\sum_{j} T^{j} \omega_{B}^{j}\left(e_{k}\right)
\end{aligned}
$$

e

$$
\sum_{\gamma} \nu^{\gamma} \omega_{\gamma}^{B}=-\sum_{\gamma} T^{\gamma} \omega_{B}^{\gamma}\left(e_{k}\right),
$$


segue que

$$
d T^{B}\left(e_{k}\right)-\sum_{j} T^{j} \omega_{B}^{j}\left(e_{k}\right)-\sum_{\gamma} T^{\gamma} \omega_{B}^{\gamma}\left(e_{k}\right)=0
$$

Tomemos a matriz de 1 -formas $\Omega$ definida por

$$
\Omega=\left(\omega_{C}^{B}\right) \in \mathcal{M}_{m+2}(\mathbb{R}),
$$

onde $0 \leq B, C \leq m+1$.

Lema 2.2.5. Temos

$$
d \Omega+\Omega \wedge \Omega=0
$$

na qual $d \Omega=\left(d \omega_{C}^{B}\right)$ e $\Omega \wedge \Omega=\left(\sum_{D} \omega_{D}^{B} \wedge \omega_{C}^{D}\right)$.

Demonstração. Escreva $\Psi=d \Omega+\Omega \wedge \Omega$. Mostraremos que $\Psi_{C}^{B}=0$. Pela equação (2.8) temos que

$$
\Psi_{j}^{i}=d \omega_{j}^{i}+\sum_{C} \omega_{C}^{i} \wedge \omega_{j}^{C}=\varepsilon\left(\omega^{i} \wedge \omega^{j}+T^{i} \omega^{j} \wedge \eta-T^{j} \omega^{i} \wedge \eta\right)+\omega_{0}^{i} \wedge \omega_{j}^{0} .
$$

Além disso,

$$
\begin{aligned}
\omega_{0}^{i} \wedge \omega_{j}^{0} & =\left(\omega^{i}-T^{i} \eta\right) \wedge \varepsilon\left(T^{j} \eta-\omega^{j}\right) \\
& =\varepsilon\left(\omega^{i} \wedge T^{j} \eta-\omega^{i} \wedge \omega^{j}-T^{i} \eta \wedge T^{j} \eta+T^{i} \eta \wedge \omega^{j}\right) \\
& =-\varepsilon\left(\omega^{i} \wedge \omega^{j}+T^{i} \omega^{j} \wedge \eta-T^{j} \omega^{i} \wedge \eta\right),
\end{aligned}
$$

$\log \Psi_{j}^{i}=0$

Por (2.9), temos que

$$
\Psi_{j}^{\alpha}=d \omega_{j}^{\alpha}+\sum_{C} \omega_{C}^{\alpha} \wedge \omega_{j}^{C}=\varepsilon \nu^{\alpha} \omega^{j} \wedge \eta+\omega_{0}^{\alpha} \wedge \omega_{j}^{0}
$$

Mas

$$
\begin{aligned}
\omega_{0}^{\alpha} \wedge \omega_{j}^{0} & =-\nu^{\alpha} \eta \wedge \varepsilon\left(T^{j} \eta-\omega^{j}\right) \\
& =-\varepsilon \nu^{\alpha} \eta \wedge T^{j} \eta+\nu^{\alpha} \eta \wedge \omega^{j} \\
& =-\varepsilon \nu^{\alpha} \omega^{j} \wedge \eta,
\end{aligned}
$$

$\operatorname{logo} \Psi_{j}^{\alpha}=0$. Note que

$$
\Psi_{\alpha}^{j}=d \omega_{\alpha}^{j}+\sum_{C} \omega_{C}^{j} \wedge \omega_{\alpha}^{C}=-d \omega_{j}^{\alpha}-\sum_{C} \omega_{C}^{\alpha} \wedge \omega_{j}^{C}=-\Psi_{j}^{\alpha}=0 .
$$

Temos

$$
\Psi_{j}^{0}=d \omega_{j}^{0}+\sum_{k} \omega_{k}^{0} \wedge \omega_{j}^{k}+\sum_{\gamma} \omega_{\gamma}^{0} \wedge \omega_{j}^{\gamma} .
$$

Observe que pelo lema (2.2.2) e pela equação (1.6),

$$
\begin{aligned}
d \omega_{j}^{0} & =d\left(\varepsilon\left(T^{j} \eta-\omega^{j}\right)\right) \\
& =\varepsilon\left(d T^{j} \wedge \eta+T^{j} d \eta-d \omega^{j}\right) \\
& =\varepsilon d T^{j} \wedge \eta+\varepsilon \sum_{k} \omega_{k}^{j} \wedge \omega^{k} .
\end{aligned}
$$


Também temos

$$
\begin{aligned}
\sum_{k} \omega_{k}^{0} \wedge \omega_{j}^{k} & =\sum_{k} \varepsilon\left(T^{k} \eta-\omega^{k}\right) \wedge \omega_{j}^{k} \\
& =-\varepsilon \sum_{k} T^{k} \omega_{j}^{k} \wedge \eta-\varepsilon \sum_{k} \omega_{k}^{j} \wedge \omega^{k},
\end{aligned}
$$

e

$$
\sum_{\gamma} \omega_{\gamma}^{0} \wedge \omega_{j}^{\gamma}=\sum_{\gamma} \varepsilon \nu^{\gamma} \eta \wedge \omega_{j}^{\gamma}=-\varepsilon \sum_{\gamma} T^{\gamma} \omega_{j}^{\gamma} \wedge \eta .
$$

Substituindo na equação acima temos

$$
\Psi_{j}^{0}=\varepsilon\left(d T^{j}-\sum_{C} T^{C} \omega_{j}^{C}\right) \wedge \eta
$$

e pelo lema (2.2.4) segue que

$$
\Psi_{j}^{0}=0
$$

Temos

$$
\Psi_{\alpha}^{0}=d \omega_{\alpha}^{0}+\sum_{k} \omega_{k}^{0} \wedge \omega_{\alpha}^{k}+\sum_{\gamma} \omega_{\gamma}^{0} \wedge \omega_{\alpha}^{\gamma} .
$$

Observe que pelo lema (2.2.2),

$$
d \omega_{\alpha}^{0}=d\left(\varepsilon \nu^{\alpha} \eta\right)=\varepsilon d \nu^{\alpha} \wedge \eta+\varepsilon \nu^{\alpha} \wedge d \eta=\varepsilon d \nu^{\alpha} \wedge \eta .
$$

Temos que, pela equação (2.6),

$$
\begin{aligned}
\sum_{k} \omega_{k}^{0} \wedge \omega_{\alpha}^{k} & =\sum_{k} \varepsilon\left(T^{k} \eta-\omega^{k}\right) \wedge \omega_{\alpha}^{k} \\
& =-\varepsilon \sum_{k} T^{k} \omega_{\alpha}^{k} \wedge \eta-\varepsilon \sum_{k} \omega_{k}^{\alpha} \wedge \omega^{k} \\
& =-\varepsilon \sum_{k} T^{k} \omega_{\alpha}^{k} \wedge \eta
\end{aligned}
$$

e também

$$
\sum_{\gamma} \omega_{\gamma}^{0} \wedge \omega_{\alpha}^{\gamma}=\sum_{\gamma} \varepsilon \nu^{\gamma} \eta \wedge \omega_{\alpha}^{\gamma}=-\varepsilon \sum_{\gamma} T^{\gamma} \omega_{\alpha}^{\gamma} \wedge \eta .
$$

Substituindo na equação acima temos

$$
\Psi_{j}^{0}=\varepsilon\left(d T^{\alpha}-\sum_{C} T^{C} \omega_{\alpha}^{C}\right) \wedge \eta,
$$

e novamente pelo lema (2.2.4) segue que

$$
\Psi_{\alpha}^{0}=0 .
$$

Note que

$$
\Psi_{0}^{B}=d \omega_{0}^{B}+\sum_{C} \omega_{C}^{B} \wedge \omega_{0}^{C}=-\varepsilon d \omega_{j}^{\alpha}-\varepsilon \sum_{C} \omega_{C}^{0} \wedge \omega_{B}^{C}=-\Psi_{B}^{0}=0
$$

Pela proposição (2.10) temos

$$
\Psi_{\beta}^{\alpha}=d \omega_{\beta}^{\alpha}+\omega_{0}^{\alpha} \wedge \omega_{\beta}^{0}+\sum_{k} \omega_{k}^{\alpha} \wedge \omega_{\beta}^{k}+\sum_{\gamma} \omega_{\gamma}^{\alpha} \wedge \omega_{\beta}^{\gamma}=\omega_{0}^{\alpha} \wedge \omega_{\beta}^{0}
$$


Mas

$$
\omega_{0}^{\alpha} \wedge \omega_{\beta}^{0}=-\nu^{\alpha} \eta \wedge \varepsilon \nu^{\beta} \eta=0
$$

$\operatorname{logo} \Psi_{\beta}^{\alpha}=0$.

Como

$$
\Psi_{0}^{0}=d \omega_{0}^{0}+\sum_{C} \omega_{C}^{0} \wedge \omega_{0}^{C}=0
$$

concluímos que $d \Omega+\Omega \wedge \Omega=0$

Definimos agora, para cada $x \in M$, o conjunto

$$
\mathcal{Z}(x)=\left\{Z \in S O^{+}\left(\mathbb{E}^{m+2}\right) ; Z_{C}^{m+1}=T^{C}(x)\right\} .
$$

Lema 2.2.6. Para todo $x \in M, \mathcal{Z}(x)$ é uma variedade diferenciável de dimensão $\frac{m(m+1)}{2}$.

Demonstração. Seja $\mathbb{S}\left(\mathbb{E}^{m+2}\right)=\left\{x \in \mathbb{E}^{m+2} ;\langle x, x\rangle_{\varepsilon}=1\right\}$ e $F: S O^{+}\left(\mathbb{E}^{m+2}\right) \rightarrow \mathbb{S}\left(\mathbb{E}^{m+2}\right)$ dada por $F(Z)=\left(Z_{C}^{m+1}\right)_{C}$, ou seja, $F(Z)$ é a última linha de $Z$. Vamos primeiramente mostrar que $F$ é uma submersão. Sejam $Z \in S O^{+}\left(\mathbb{E}^{m+2}\right)$ e $\zeta \in T_{Z} S O^{+}\left(\mathbb{E}^{m+2}\right)$ e vamos calcular $d F_{Z}(\zeta)$. Defina $\bar{F}: \mathcal{M}_{m+2}(\mathbb{R}) \rightarrow \mathbb{R}^{m+2}$ dada por $\bar{F}(Z)=\left(Z_{C}^{m+1}\right)_{C}$. Note que $\left.\bar{F}\right|_{S O^{+}\left(\mathbb{E}^{m+2}\right)}=F$. Como $\bar{F}$ é linear em $\mathcal{M}_{m+2}(\mathbb{R})$, temos que para $A \in T_{Z} \mathcal{M}_{m+2}(\mathbb{R}), d \bar{F}_{Z}(A)=\left(A_{C}^{m+1}\right)_{C}$. Como $\bar{F}$ é extensão de $F$ concluímos que $d F_{Z}(\zeta)=\left(\zeta_{C}^{m+1}\right)_{C}$.

Mostraremos agora que $d F_{Z}$ é sobrejetora. Sabemos que se $\zeta \in T_{Z} S O^{+}\left(\mathbb{E}^{m+2}\right)$, então $\zeta=Z X$, para algum $X \in T_{I} S O^{+}\left(\mathbb{E}^{m+2}\right)=\mathfrak{s o}\left(E^{m+2}\right)$, ou seja, $X^{t} G+G X=0$ e portanto $X=-G X^{t} G$. Então temos

$$
X_{j}^{i}=-X_{i}^{j}
$$

Para os demais índices, observe que

$$
\begin{aligned}
X_{\alpha}^{i} & =-\left(G X^{t} G\right)_{\alpha}^{i}=-\sum_{C} G_{C}^{i}\left(X^{t} G\right)_{\alpha}^{C}=-\left(X^{t} G\right)_{\alpha}^{i} \\
& =-\sum_{C}\left(X^{t}\right)_{C}^{i} G_{\alpha}^{C}=-X_{i}^{\alpha}
\end{aligned}
$$

Analogamente,

$$
X_{\alpha}^{\beta}=-X_{\beta}^{\alpha}
$$

Também temos

$$
\begin{aligned}
X_{B}^{0}= & =-\left(G X^{t} G\right)_{B}^{0}=-\sum_{C} G_{C}^{0}\left(X^{t} G\right)_{B}^{C}=-\varepsilon\left(X^{t} G\right)_{B}^{0} \\
& =-\varepsilon \sum_{C}\left(X^{t}\right)_{C}^{0} G_{B}^{C}=-\varepsilon X_{0}^{B} G_{B}^{B} \\
& \Rightarrow X_{0}^{0}=0, \quad X_{i}^{0}=-\varepsilon X_{0}^{i}, \quad X_{\alpha}^{0}=-\varepsilon X_{0}^{\alpha}
\end{aligned}
$$

Sabemos que $d F_{Z}(Z X)=\left((Z X)_{C}^{m+1}\right)_{C}=\left(\sum_{B} Z_{B}^{m+1} X_{C}^{B}\right)_{C}$. Observe que para cada índice $C$ temos: 
- Se $C=0$,

$$
\begin{aligned}
(Z X)_{0}^{m+1} & =\sum_{B} Z_{B}^{m+1} X_{0}^{B} \\
& =Z_{0}^{m+1} X_{0}^{0}+\sum_{j} Z_{j}^{m+1} X_{0}^{j}+\sum_{\gamma} Z_{\gamma}^{m+1} X_{0}^{\gamma} \\
& =Z_{0}^{m+1}\left(-\varepsilon X_{0}^{0}\right)+\sum_{j} Z_{j}^{m+1}\left(-\varepsilon X_{j}^{0}\right)+\sum_{\gamma} Z_{\gamma}^{m+1}\left(-\varepsilon X_{\gamma}^{0}\right) \\
& =-\varepsilon \sum_{B} X_{B}^{0} Z_{B}^{m+1} .
\end{aligned}
$$

- Se $0<C \leq m+1$,

$$
\begin{aligned}
(Z X)_{C}^{m+1} & =\sum_{B} Z_{B}^{m+1} X_{C}^{B} \\
& =Z_{0}^{m+1} X_{C}^{0}+\sum_{j} Z_{j}^{m+1} X_{C}^{j}+\sum_{\gamma} Z_{\gamma}^{m+1} X_{C}^{\gamma} \\
& =-\varepsilon Z_{0}^{m+1} X_{0}^{C}-\sum_{j} X_{j}^{C} Z_{j}^{m+1}-\sum_{\gamma} X_{\gamma}^{C} Z_{\gamma}^{m+1} .
\end{aligned}
$$

Seja $v \in T_{F(Z)} \mathbb{S}\left(\mathbb{E}^{m+2}\right)$. Escrevendo $v=\left(v_{0}, \ldots, v_{m+1}\right)$, temos

$$
\varepsilon v_{0} Z_{0}^{m+1}+\sum_{j} v_{j} Z_{j}^{m+1}+\sum_{\gamma} v_{\gamma} Z_{\gamma}^{m+1}=0
$$

Vamos mostrar que existe $X \in T_{I} S O^{+}\left(\mathbb{E}^{m+2}\right)$ tal que $d F_{Z}(Z X)=v$. Como $Z \in S O^{+}\left(\mathbb{E}^{m+2}\right)$, existe $B$ tal que $Z_{B}^{m+1} \neq 0$. Dividiremos em dois casos.

1. Se $B=0$, então

$$
v_{0}=\varepsilon\left(\frac{-\sum_{j} v_{j} Z_{j}^{m+1}-\sum_{\gamma} v_{\gamma} Z_{\gamma}^{m+1}}{Z_{0}^{m+1}}\right)
$$

Para cada índice $D$, com $D \neq B$, definimos a matriz $X(D)$ por $(X(D))_{D}^{0}=1,(X(D))_{0}^{D}=$ $-\varepsilon(X(D))_{D}^{0}=-\varepsilon$ e os demais termos nulos. Note que para cada $D, X(D) \in T_{I} S O^{+}\left(\mathbb{E}^{m+2}\right)$. Pelas relações (2.14) e (2.15), temos que

$$
d F_{Z}(Z X(D))=\left((Z X(D))_{C}^{m+1}\right)_{C}=\left(-\varepsilon Z_{D}^{m+1}, 0, \ldots, 0, Z_{0}^{m+1}, 0 \ldots, 0\right),
$$

onde $Z_{0}^{m+1}$ é o D-ésimo termo.

Tomando

$$
X=\sum_{D} \frac{v_{D}}{Z_{0}^{m+1}} X(D)
$$

segue que $X \in T_{I} S O^{+}\left(\mathbb{E}^{m+2}\right)$ e

$$
d F_{Z}(Z X)=\left(\varepsilon\left(\frac{-\sum_{j} v_{j} Z_{j}^{m+1}-\sum_{\gamma} v_{\gamma} Z_{\gamma}^{m+1}}{Z_{0}^{m+1}}\right), v_{1}, \ldots, v_{m+1}\right)=v .
$$

2. Se $B \neq 0$, então

$$
v_{B}=\left(\frac{-\varepsilon v_{0} Z_{0}^{m+1}-v_{1} Z_{1}^{m+1}-\ldots-\widehat{v_{B} Z_{B}^{m+1}}-\ldots-v_{m+1} Z_{m+1}^{m+1}}{Z_{B}^{m+1}}\right)
$$


Para cada índice $D$, com $D \neq B$ e $D \neq 0$, definimos a matriz $X(D)$ por $(X(D))_{D}^{i}=1$, $(X(D))_{i}^{D}=-1$ e os demais termos nulos, se $D \neq 0$, e $X(0)$ por $X(0)_{0}^{i}=1, X(0)_{i}^{0}=$ $-\varepsilon X(0)_{0}^{i}=-\varepsilon$ e os demais termos nulos. Note que para cada $D, X(D) \in T_{I} S O^{+}\left(\mathbb{E}^{m+2}\right)$.

Pelas relações (2.14) e (2.15) acima, temos que

$$
d F_{Z}(Z X(0))=\left((Z X(0))_{C}^{m+1}\right)_{C}=\left(Z_{B}^{m+1}, 0, \ldots, 0,-\varepsilon Z_{0}^{m+1}, 0 \ldots, 0\right),
$$

onde $-\varepsilon Z_{0}^{m+1}$ é o B-ésimo termo, e

$$
d F_{Z}(Z X(D))=\left((Z X(D))_{C}^{m+1}\right)_{C}=\left(0, \ldots, 0, Z_{B}^{m+1}, 0 \ldots, 0,-Z_{D}^{m+1}, 0, \ldots, 0\right),
$$

onde $Z_{B}^{m+1}$ é o D-ésimo termo e $-Z_{D}^{m+1}$ é o B-ésimo termo.

Tomando

$$
X=\frac{v_{0}}{Z_{B}^{m+1}} X(0)+\ldots+\frac{v_{B-1}}{Z_{B}^{m+1}} X(B-1)+\frac{v_{B+1}}{Z_{B}^{m+1}} X(B+1)+\ldots+\frac{v_{m+1}}{Z_{B}^{m+1}} X(m+1),
$$

segue que $X \in T_{I} S O^{+}\left(\mathbb{E}^{m+2}\right)$ e $d F_{Z}(Z X)=v$.

Portanto $F$ é submersão. Então, se $x \in M$, o conjunto $F^{-1}\left(T^{0}(x), \ldots, T^{m+1}(x)\right)=\mathcal{Z}(x)$ é uma variedade diferenciável. Além disso, como $\operatorname{dim}\left(S O^{+}\left(\mathbb{E}^{m+2}\right)\right)=\frac{(m+1)(m+2)}{2} \operatorname{edim}\left(\mathbb{S}\left(\mathbb{E}^{m+2}\right)\right)=m+1$, temos que $\operatorname{dim}(\mathcal{Z}(x))=\frac{(m+1)(m+2)}{2}-(m+1)=\frac{m(m+1)}{2}$.

Vamos agora demonstrar a seguinte proposição, que será central para a demonstração do teorema principal deste capítulo. Note que esta proposição é motivada pelo que fizemos na seção 1.3.

Proposição 2.2.7. Assuma que as equações de compatibilidade para $\mathbb{Q}_{\varepsilon}^{m} \times \mathbb{R}$ estão satisfeitas. Seja $x_{0} \in M$ e $P_{0} \in \mathcal{Z}\left(x_{0}\right)$. Então existe uma vizinhança $U_{1}$ de $x_{0}$ e uma única aplicação $P: U_{1} \longrightarrow$ $S O^{+}\left(\mathbb{E}^{m+2}\right)$ tal que

$$
\begin{gathered}
P^{-1} d P=\Omega ; \\
\forall x \in U_{1}, P(x) \in \mathcal{Z}(x) ; \\
P\left(x_{0}\right)=P_{0} .
\end{gathered}
$$

Demonstração. Vamos resolver a equação diferencial $P^{-1} d P=\Omega$ com condição inicial $P\left(x_{0}\right)=P_{0}$.

Primeiramente vamos construir uma aplicação $Y: U \rightarrow S O^{+}\left(\mathbb{E}^{m+2}\right)$ diferenciável tal que $Y(x) \in$ $\mathcal{Z}(x)$, para todo $x \in U$, onde $U$ é uma vizinhança coordenada de $x_{0}$. Seja $V$ uma vizinhança coordenada de $x_{0}$ em $M$. Sejam $t_{0}, \ldots t_{m}$ vetores em $\mathbb{E}^{m+2}$ tais que $\left\{T^{C}\left(x_{0}\right), t_{0}, \ldots t_{m}\right\}$ forma uma base ortonormal para $\mathbb{E}^{m+2}$ e

$$
\operatorname{det}\left[\begin{array}{c}
t_{0} \\
\vdots \\
t_{m} \\
T^{C}\left(x_{0}\right)
\end{array}\right]=1
$$

onde $T^{C}(x)$ denota o vetor $\left(T^{0}(x), \ldots, T^{m+1}(x)\right)$. Então existe uma vizinhança $U$ suficientemente pequena, $U \subset V$ tal que $\left\{T^{C}(x), t_{0}, \ldots t_{m}\right\}$ é um conjunto de vetores linearmente independente em $\mathbb{E}^{m+2}$, para todo $x \in U$. Aplicando o processo de Gram-Schmidt neste conjunto para cada $x \in U$, obtemos vetores $t_{0}(x), \ldots t_{m}(x)$ definidos em $U$, com $t_{0}\left(x_{0}\right)=t_{0}, \ldots, t_{m}\left(x_{0}\right)=t_{m}$, tais que $\left\{T^{C}(x), t_{0}(x), \ldots t_{m}(x)\right\}$ é base ortonormal para $\mathbb{E}^{m+2}$ para cada $x \in U$. Definimos entao $Y: U \rightarrow$ $S O^{+}\left(\mathbb{E}^{m+2}\right)$ por

$$
Y(x)=\left[\begin{array}{c}
t_{0}(x) \\
\vdots \\
t_{m}(x) \\
T^{C}(x)
\end{array}\right] .
$$


Note que $Y(x) \in \mathcal{Z}(x)$, para todo $x \in U$.

Mostraremos agora que para $x \in U, Z \in \mathcal{Z}(x)$ se e somente se

$$
Z Y^{-1}(x)=\left[\begin{array}{cc}
B & 0 \\
0 & 1
\end{array}\right]
$$

para algum $B \in S O^{+}\left(\mathbb{E}^{(m+1)}\right)$.

Suponha que $Z \in \mathcal{Z}(x)$. Como $Y(x)$ pertence ao grupo $S O^{+}\left(\mathbb{E}^{m+2}\right)$, temos que $Y^{-1}(x) \in$ $S O^{+}\left(\mathbb{E}^{m+2}\right)$. Além disso, $\mathcal{Z} \subset S O^{+}\left(\mathbb{E}^{m+2}\right)$, e temos $Z Y^{-1}(x) \in S O^{+}\left(\mathbb{E}^{m+2}\right)$. Podemos escrever

$$
Z Y^{-1}(x)=\left[\begin{array}{cc}
B_{(m+1) \times(m+1)} & A_{(m+1) \times 1} \\
C_{1 \times(m+1)} & D_{1 \times 1}
\end{array}\right],
$$

e basta verificar que $A=0, C=0$ e $D=1$. Note que $\left(Y^{-1}\right)(x)^{t} G Y^{-1}(x)=G \Rightarrow Y^{-1}(x)=$ $G Y^{t}(x) G$, logo a última coluna de $Y^{-1}(x)$ é $\left(-T^{0}(x), T^{1}(x), \ldots, T^{m+1}(x)\right)=\left(0, T^{1}(x), \ldots, T^{m+1}(x)\right)$. Observe que a última linha de $Z$ também é $\left(0, T^{1}(x), \ldots, T^{m+1}(x)\right)$, e como $\left(T^{1}\right)^{2}+\ldots+\left(T^{m+1}\right)^{2}=$ $\|T\|^{2}+\sum_{\alpha}\left(\nu^{\alpha}\right)^{2}=1$, temos $D=1$. Agora, como $Z \in S O^{+}\left(\mathbb{E}^{m+2}\right)$, as linhas de $Z$ são ortogonais com relação à métrica de $\mathbb{E}^{m+2}$, logo as $m+1$ primeiras linhas de $Z$ são ortogonais à última coluna de $Y^{-1}(x)$, e segue que $A=0$. Como $Y^{-1}(x) \in S O^{+}\left(\mathbb{E}^{m+2}\right)$, as colunas de $Y^{-1}(x)$ também são ortogonais com relação à métrica de $\mathbb{E}^{m+2}$, e portanto as $m+1$ primeiras colunas de $Y^{-1}(x)$ são ortogonais à última linha de $Z$, e segue que $C=0$, e temos o que queríamos. Claramente $B \in S O^{+}\left(\mathbb{E}^{m+1}\right)$.

Reciprocamente, seja $B \in S O^{+}\left(\mathbb{E}^{m+1}\right)$ e

$$
Z=\left[\begin{array}{cc}
B & 0 \\
0 & 1
\end{array}\right] Y(x)
$$

Como $B \in S O^{+}\left(\mathbb{E}^{m+1}\right)$, segue que

$$
\left[\begin{array}{cc}
B & 0 \\
0 & 1
\end{array}\right] \in S O^{+}\left(\mathbb{E}^{m+2}\right)
$$

Como $Y(x) \in S O^{+}\left(\mathbb{E}^{m+2}\right)$, temos que $Z \in S O^{+}\left(\mathbb{E}^{m+2}\right)$. Note agora que a última linha de $Z$ é igual a última linha de $Y(x)$, ou seja, é igual a $\left(0, T^{1}(x), \ldots, T^{m+1}(x)\right), \log 0 Z \in \mathcal{Z}(x)$.

Definimos o conjunto:

$$
\mathcal{F}=\left\{(x, Z) \in U \times S O^{+}\left(\mathbb{E}^{m+2}\right) ; Z \in \mathcal{Z}(x)\right\} .
$$

Vamos mostrar que $\mathcal{F}$ é uma variedade diferenciável de dimensão $n+\frac{m(m+1)}{2}$.

Como $S O^{+}\left(\mathbb{E}^{m+1}\right)$ é uma variedade diferenciável de dimensão $\frac{m(m+1)}{2}$, se $\mathcal{A}$ é um atlas para $S O^{+}\left(\mathbb{E}^{m+1}\right)$, para cada $(\phi, W) \in \mathcal{A}$ seja

$$
\Phi(v)=\left[\begin{array}{cc}
\phi(v) & 0 \\
0 & 1
\end{array}\right]
$$

com inversa

$$
\Phi^{-1}\left(\left[\begin{array}{cc}
B & 0 \\
0 & 1
\end{array}\right]\right)=\phi^{-1}(B) .
$$

Pelo que vimos acima, se $x \in U$, então $\Phi(v) Y(x) \in \mathcal{Z}(x)$ e podemos definir a aplicação $F_{\phi}$ : $U \times W \subset \mathbb{R}^{n} \times \mathbb{R}^{\frac{m(m+1)}{2}} \rightarrow \mathcal{F}$ dada por $F_{\phi}(x, v)=(x, \Phi(v) Y(x))$. Se $\left(\phi_{1}, W_{1}\right),\left(\phi_{2}, W_{2}\right) \in \mathcal{A}$, com $F_{\phi_{1}}\left(U \times W_{1}\right) \cap F_{\phi_{2}}\left(U \times W_{2}\right) \neq \emptyset$, então

$$
F_{\phi_{2}}^{-1} \circ F_{\phi_{1}}: F_{\phi_{1}}^{-1}\left(F_{\phi_{1}}\left(U \times W_{1}\right) \cap F_{\phi_{2}}\left(U \times W_{2}\right)\right) \rightarrow F_{\phi_{2}}^{-1}\left(F_{\phi_{1}}\left(U \times W_{1}\right) \cap F_{\phi_{2}}\left(U \times W_{2}\right)\right)
$$


é diferenciável. De fato, se $(x, v) \in F_{\phi_{1}}^{-1}\left(F_{\phi_{1}}\left(U \times W_{1}\right) \cap F_{\phi_{2}}\left(U \times W_{2}\right)\right)$, então $F_{\phi_{2}}^{-1} \circ F_{\phi_{1}}(x, v)=$ $F_{\phi_{2}}^{-1}\left(x, \Phi_{1}(v) Y(x)\right)=\left(x, \Phi_{2}^{-1}\left(\Phi_{1}(v) Y(x) Y^{-1}(x)\right)\right)=\left(x, \Phi_{2}^{-1}\left(\Phi_{1}(v)\right)\right)=\left(x, \phi_{2}^{-1}\left(\phi_{1}(v)\right)\right)$. Desta forma, a partir de $\mathcal{A}$ podemos construir um atlas para $\mathcal{F}$, portanto $\mathcal{F}$ é uma variedade diferenciável de dimensão $n$. Além disso, temos que

$$
T_{(x, Z)} \mathcal{F}=\left\{(u, \zeta) \in T_{x} U \times T_{Z} S O^{+}\left(\mathbb{E}^{m+2}\right) ; \zeta_{C}^{m+1}=\left(d T^{C}\right)_{x}(u)\right\} .
$$

Denote por $\pi_{1}$ e por $\pi_{2}$ as projeções

$$
\begin{aligned}
& \pi_{1}: U \times S O^{+}\left(\mathbb{E}^{m+2}\right) \rightarrow U, \\
& \pi_{2}: U \times S O^{+}\left(\mathbb{E}^{m+2}\right) \rightarrow S O^{+}\left(\mathbb{E}^{m+2}\right) .
\end{aligned}
$$

Seja $g: G L\left(\mathbb{E}^{m+2}\right) \rightarrow G L\left(\mathbb{E}^{m+2}\right)$ dada por $g(A)=A^{-1}$. Definimos a seguinte matriz de 1 -formas em $\mathcal{F}$ :

$$
\theta=\left(g \circ \pi_{2}\right)\left[d\left(a_{J}^{I} \circ \pi_{2}\right)\right]_{J}^{I}-\pi_{1}^{*} \Omega,
$$

na qual $a_{j}^{i}(Z)=Z_{j}^{i}$. Então, como

$$
\begin{aligned}
{\left[d\left(a_{j}^{i} \circ \pi_{2}\right)\right]_{j}^{i} } & =\left[d a_{j}^{i} \circ d \pi_{2}\right]_{j}^{i} \\
& =\left[\left(d \pi_{2}\right)_{j}^{i}\right]_{j}^{i} \\
& =d \pi_{2},
\end{aligned}
$$

temos que $\theta_{(x, Z)}: T_{(x, Z)} \mathcal{F} \rightarrow \mathcal{M}_{m+2}(\mathbb{R})$ é dada por

$$
\theta_{(x, Z)}(u, \zeta)=\left(g \circ \pi_{2}\right)(x, Z)\left[d\left(a_{j}^{i} \circ \pi_{2}\right)_{(x, Z)}(u, \zeta)\right]_{j}^{i}-\left(\pi_{1}^{*} \Omega\right)_{(x, Z)}(u, \zeta)=Z^{-1} \zeta-\Omega_{x}(u) .
$$

Note que

$$
\begin{aligned}
d\left(g \circ \pi_{2}\right) & =d g_{\pi_{2}} \circ d \pi_{2} \\
& =-\left(g \circ \pi_{2}\right) d \pi_{2}\left(g \circ \pi_{2}\right) .
\end{aligned}
$$

Como $d^{2}=0$ e $d$ comuta com o pullback, temos

$$
d \theta=-\left(g \circ \pi_{2}\right) d \pi_{2} \wedge\left(g \circ \pi_{2}\right) d \pi_{2}-\pi_{1}^{*} d \Omega,
$$

e como $\pi_{2}(x, Z)=Z$, vamos denotar $\pi_{2}=Z$. Denotaremos também $\pi_{1}^{*} \Omega=\Omega$. Desta forma podemos escrever

$$
\begin{aligned}
& \theta=Z^{-1} d Z-\Omega, \\
& d \theta=-Z^{-1} d Z \wedge Z^{-1} d Z-d \Omega .
\end{aligned}
$$

Para cada ponto $(x, Z) \in \mathcal{F}$, definimos o espaço

$$
\mathcal{D}_{(x, Z)}=\operatorname{ker} \theta(x, Z)
$$

. Vamos mostrar que $\mathcal{D}_{(x, Z)}$ tem dimensão $n$. Observe que

- $\Omega \in \mathfrak{s o}\left(\mathbb{E}^{m+2}\right)$;

- Se $Z \in \mathcal{Z}(x)$ e $\zeta \in T_{Z} S O^{+}\left(\mathbb{E}^{m+2}\right)$, com $\zeta_{C}^{m+1}=d T_{x}^{C}(u), u \in U$, então $\zeta=Z X$, com $X \in \mathfrak{s o}\left(\mathbb{E}^{m+2}\right), \log \left(Z^{-1} \zeta\right)^{t} G+G\left(Z^{-1} \zeta\right)=\left(Z^{-1} Z X\right)^{t} G+G\left(Z^{-1} Z X\right)=X^{t} G+G X=0$, $\operatorname{logo} Z^{-1} \zeta \in \mathfrak{s o}\left(\mathbb{E}^{m+2}\right)$.

Portanto $\theta \in \mathfrak{s o}\left(\mathbb{E}^{m+2}\right)$. Além disso, temos que pelo lema (2.2.4),

$$
(Z \theta)_{C}^{m+1}=(\zeta-Z \Omega)_{C}^{m+1}=\zeta_{C}^{m+1}-\sum_{B} Z_{B}^{m+1} \omega_{C}^{B}=d T^{C}-\sum_{B} T^{B} \omega_{C}^{B}=0,
$$


portanto imagem de $\theta_{(x, Z)}$ está no espaço

$$
\mathcal{H}=\left\{h \in \mathfrak{s o}\left(\mathbb{E}^{m+2}\right) ;(Z H)_{C}^{m+1}=0\right\} .
$$

Então $\operatorname{dim}\left(\operatorname{Im}\left(\theta_{(x, Z)}\right)\right) \leq \operatorname{dim}(\mathcal{H})$. Note que se $H \in \mathcal{H}$, entao $Z H \in T_{Z} S O^{+}\left(\mathbb{E}^{m+2}\right)$, e como $(Z H)_{C}^{m+1}=0=d T_{x}^{m+1}(0)$, então $\{(0, Z H) ; H \in \mathcal{H}\} \subset T_{(x, Z)} \mathcal{F}$. Como $\theta_{(x, Z)}(0, Z H)=H$, temos $\mathcal{H} \subset \operatorname{Im}\left(\theta_{(x, Z)}\right) \Rightarrow \operatorname{dim}(\mathcal{H}) \leq \operatorname{dim}\left(\operatorname{Im}\left(\theta_{(x, Z)}\right)\right), \operatorname{logo} \operatorname{dim}(\mathcal{H})=\operatorname{dim}\left(\operatorname{Im}\left(\theta_{(x, Z)}\right)\right)$. Basta agora calcular $\operatorname{dim}(\mathcal{H})$. Pelo que vimos na demonstração do lema $(2.2 .6)$, a aplicação $F: S O^{+}\left(\mathbb{E}^{m+2}\right) \rightarrow \mathbb{S}\left(\mathbb{E}^{m+2}\right)$ dada por $F(Z)=\left(Z_{C}^{m+1}\right)_{C}$ é uma submersão. Note que $H \in \mathcal{H} \Leftrightarrow Z H \in \operatorname{ker}\left(d F_{Z}\right)$, daí segue que $\operatorname{dim}\left(\operatorname{Im}\left(\theta_{(x, Z)}\right)\right)=\operatorname{dim}(\mathcal{H})=\operatorname{dim}\left(\operatorname{ker}\left(d F_{Z}\right)\right)=\frac{m(m+1)}{2}$. Pelo teorema do Núcleo e Imagem temos

$$
\operatorname{dim}\left(\operatorname{Im}\left(\theta_{(x, Z)}\right)\right)+\operatorname{dim}\left(\operatorname{ker}\left(\theta_{(x, Z)}\right)\right)=\operatorname{dim} \mathcal{F} \Rightarrow \operatorname{dim} \mathcal{D}_{(x, Z)}=n
$$

Provaremos agora que $\mathcal{D}$ é uma distribuição involutiva. Temos que

$$
\begin{aligned}
d \theta & =-Z^{-1} d Z \wedge Z^{-1} d Z-d \Omega \\
& =-(\theta+\Omega) \wedge(\theta+\Omega)-d \Omega \\
& =-\theta \wedge \theta-\theta \wedge \Omega-\Omega \wedge \theta-\Omega \wedge \Omega-d \Omega .
\end{aligned}
$$

Pelo lema (2.2.5), segue que

$$
d \theta=-\theta \wedge \theta-\theta \wedge \Omega-\Omega \wedge \theta
$$

Sejam $\xi_{1}, \xi_{2} \in \mathcal{D}$. Então $\theta\left(\xi_{1}\right)=\theta\left(\xi_{2}\right)=0, \log \mathrm{o}$

$$
\begin{aligned}
d \theta\left(\xi_{1}, \xi_{2}\right) & =-\theta \wedge \theta\left(\xi_{1}, \xi_{2}\right)-\theta \wedge \Omega\left(\xi_{1}, \xi_{2}\right)-\Omega \wedge \theta\left(\xi_{1}, \xi_{2}\right) \\
& =-\theta\left(\xi_{1}\right) \theta\left(\xi_{2}\right)+\theta\left(\xi_{2}\right) \theta\left(\xi_{1}\right)-\theta\left(\xi_{1}\right) \Omega\left(\xi_{2}\right)+\theta\left(\xi_{2}\right) \Omega\left(\xi_{1}\right)-\Omega\left(\xi_{1}\right) \theta\left(\xi_{2}\right)+\Omega\left(\xi_{2}\right) \theta\left(\xi_{1}\right) \\
& =0 .
\end{aligned}
$$

Além disso,

$$
\begin{aligned}
d \theta\left(\xi_{1}, \xi_{2}\right) & =\xi_{1} \theta\left(\xi_{2}\right)-\xi_{2} \theta\left(\xi_{1}\right)-\theta\left(\left[\xi_{1}, \xi_{2}\right]\right) \\
& =-\theta\left(\left[\xi_{1}, \xi_{2}\right]\right)
\end{aligned}
$$

portanto

$$
\theta\left(\left[\xi_{1}, \xi_{2}\right]\right)=0 \Rightarrow\left[\xi_{1}, \xi_{2}\right] \in \mathcal{D}
$$

ou seja, $\mathcal{D}$ é involutiva. Pelo Teorema de Frobenius (veja [Lee12]), temos que $\mathcal{D}$ é integrável. Seja $\mathcal{P}$ a variedade integral passando por $\left(x_{0}, P_{0}\right)$. Se $\zeta \in T_{P_{0}} S O^{+}\left(\mathbb{E}^{m+2}\right)$ é tal que $(0, \zeta) \in T_{\left(x_{0}, P_{0}\right)} \mathcal{P}=$ $\mathcal{D}\left(x_{0}, P_{0}\right)$, então temos que

$$
\theta_{\left(x_{0}, P_{0}\right)}(0, \zeta)=P_{0}^{-1} \zeta-\Omega_{x_{0}}(0)=P_{0}^{-1} \zeta \Rightarrow \zeta=0,
$$

portanto

$$
T_{\left(x_{0}, P_{0}\right)} \mathcal{P} \cap\left(\{0\} \times T_{P_{0}} S O^{+}\left(\mathbb{E}^{m+2}\right)\right)=0 .
$$

Portanto, restringindo a projeção $\pi_{1}$ em $\mathcal{P}$, temos que

$$
\left.d \pi_{1_{\left(x_{0}, P_{0}\right)}}\right|_{T_{\left(x_{0}, P_{0}\right)} \mathcal{P}}: T_{\left(x_{0}, P_{0}\right)} \mathcal{P} \subset T_{x_{0}} U \times T_{P_{0}} S O^{+}\left(\mathbb{E}^{m+2}\right) \rightarrow T_{x_{0}} U
$$

é um isomorfismo, e pelo teorema da função inversa a projeção $\left.\pi_{1}\right|_{\mathcal{P}}: \mathcal{P} \rightarrow U$ admite uma inversa local, logo $\mathcal{P}$ pode ser escrita localmente como gráfico de uma função $P: U_{1} \longrightarrow S O^{+}\left(\mathbb{E}^{m+2}\right)$, onde $U_{1}$ é uma vizinhança de $x_{0}$ em $U$. Então

- Claramente $P\left(x_{0}\right)=P_{0}$;

- Como $\mathcal{P}$ é subvariedade de $\mathcal{F}, P(x) \in \mathcal{Z}(x)$; 
- Como $\theta_{(x, P)}=0$, se $v \in T_{(x, P(x))}$, com $v=\left(u, d P_{x}(u)\right)$, então $P(x)^{-1} d P_{x}(u)=\Omega_{x}(u)$.

Portanto, $P$ satisfaz as propriedades da proposição.

Vamos agora demonstrar o teorema principal deste capítulo, que chamaremos de Teorema Fundamental das Imersões. A demonstração é motivada novamente pelo que fizemos na seção 1.3, mais precisamente pela observação (1.3.2).

Teorema 2.2.8 (Teorema Fundamental das Imersões). Seja $M$ uma variedade Riemanniana simplesmente conexa de dimensão $n$, com métrica $\langle$,$\rangle , conexão \nabla$ e tensor curvatura $R$. Para $n+1 \leq$ $\alpha, \beta \leq m+1, \operatorname{sejam} A_{\alpha}: T M \rightarrow T M$ tensores simétricos em $M, \omega_{\alpha}^{\beta} 1$-formas em $M, \operatorname{com} \omega_{\beta}^{\alpha}=-\omega_{\alpha}^{\bar{\beta}}$, e $\nu^{\alpha}$ funções diferenciáveis em $M$. Seja $T$ um campo de vetores em $M$ tal que $\|T\|^{2}+\sum_{\alpha}\left(\nu^{\alpha}\right)^{2}=1$. Suponha que $\left(\langle\rangle,, A_{\alpha}, \omega_{\alpha}^{\beta}, T, \nu^{\alpha}\right)$ satisfaz as equações de compatibilidade para $\mathbb{Q}_{\varepsilon}^{m} \times \mathbb{R}$. Então existe uma imersão

$$
f: M \rightarrow \mathbb{Q}_{\varepsilon}^{m} \times \mathbb{R}
$$

tal que

1. Existem seções ortonormais $N_{\alpha}$ do fibrado normal de $f(M)$ em $\mathbb{Q}_{\varepsilon}^{m} \times \mathbb{R}$;

2. O operador forma com respeito a $N_{\alpha}$ associado a $f$ é

$$
\tilde{A}_{\alpha}=d f \circ A_{\alpha} \circ d f^{-1}
$$

3. As formas de conexão normais de $f(M)$ em $\mathbb{Q}_{\varepsilon}^{m} \times \mathbb{R}$ são $\tilde{\omega}_{\alpha}^{\beta}$ tais que

$$
f^{*} \tilde{\omega}_{\alpha}^{\beta}=\omega_{\alpha}^{\beta}
$$

4. O vetor vertical $\frac{\partial}{\partial t}$ associado a $\mathbb{R}$ satisfaz

$$
\frac{\partial}{\partial t}=d f(T)+\sum_{\gamma} \nu^{\gamma} N_{\gamma}
$$

Além disso, a imersão é única a menos de uma isometria global de $\mathbb{Q}_{\varepsilon}^{m} \times \mathbb{R}$ que preserva as orientações de $\mathbb{Q}_{\varepsilon}^{m}$ e $\mathbb{R}$.

Demonstração. Seja $x_{0} \in M, P_{0} \in \mathcal{Z}\left(x_{0}\right)$, com $\left(P_{0}\right)_{0}^{0}>0$, e $t_{0} \in \mathbb{R}$. Considere em $M$ um referencial ortonormal local $\left\{e_{i}\right\}_{i=1}^{n}$ em uma vizinhança de $x_{0}$, e use as mesmas notações. Pela Proposição (2.2.7), existe uma única aplicação $P: U_{1} \subset M \rightarrow S O^{+}\left(\mathbb{E}^{m+2}\right)$ tal que

$$
\begin{gathered}
P^{-1} d P=\Omega \\
\forall x \in U_{1}, P(x) \in \mathcal{Z}(x) \\
P\left(x_{0}\right)=P_{0}
\end{gathered}
$$

onde $U_{1}$ é uma vizinhança de $x_{0}$. Podemos assumir que $U_{1}$ é simplesmente conexo e $P_{0}^{0}>0$ em $U_{1}$ quando $U_{1}$ for suficientemente pequeno.

Definimos em $U_{1}$ as seguintes funções: $f^{0}=P_{0}^{0}, f^{1}=P_{0}^{1}, \ldots, f^{n}=P_{0}^{n}, f^{n+1}=P_{0}^{n+1}, \ldots, f^{m}=$ $P_{0}^{m}$.

Como $U_{1}$ é simplesmente conexo e $d \eta=0$, existe uma única função $f^{m+1}$ definida em $U_{1}$ tal que

$$
d f^{m+1}=\eta, \quad f^{m+1}\left(x_{0}\right)=t_{0} .
$$

Definimos agora a aplicação $f: U_{1} \rightarrow \mathbb{E}^{m+2}$ dada por

$$
f(x)=\left(f^{0}(x), \ldots, f^{m+1}(x)\right) .
$$


Como $P \in S O^{+}\left(\mathbb{E}^{m+2}\right)$ e $P_{0}^{m+1}=T^{0}=0$, temos que

$$
\varepsilon\left(f^{0}\right)^{2}+\sum_{i}\left(f^{i}\right)^{2}+\sum_{\alpha=n+1}^{m}\left(f^{\alpha}\right)^{2}=\varepsilon\left(P_{0}^{0}\right)^{2}+\sum_{i}\left(P_{0}^{i}\right)^{2}+\sum_{\alpha}\left(P_{0}^{\alpha}\right)^{2}=\varepsilon,
$$

e como $f^{0}=P_{0}^{0}>0$ podemos concluir que $\left(f^{0}, \ldots, f^{m}\right) \in \mathbb{Q}_{\varepsilon}^{m}, \operatorname{logo} f \in \mathbb{Q}_{\varepsilon}^{m} \times \mathbb{R}$. Como $P^{-1} d P=\Omega$, temos $d P=P \Omega$, logo, para $B<m+1$,

$$
\begin{aligned}
d f^{B}\left(e_{k}\right) & =P_{0}^{B} \omega_{0}^{0}\left(e_{k}\right)+\sum_{j} P_{j}^{B} \omega_{0}^{j}\left(e_{k}\right)+\sum_{\alpha} P_{\alpha}^{B} \omega_{0}^{\alpha}\left(e_{k}\right) \\
& =\sum_{j} P_{j}^{B}\left(\omega^{j}\left(e_{k}\right)-T^{j} \eta\left(e_{k}\right)\right)+\sum_{\alpha} P_{\alpha}^{B}\left(-\nu^{\alpha} \eta\left(e_{k}\right)\right) \\
& =\sum_{j} P_{j}^{B}\left(\delta_{j k}-T^{j} T^{k}\right)-\sum_{\alpha} P_{\alpha}^{B} T^{\alpha} T^{k} \\
& =P_{k}^{B}-T^{k}\left(\sum_{j} P_{j}^{B} P_{j}^{m+1}+\sum_{\alpha} P_{\alpha}^{B} P_{j}^{m+1}\right) \\
& =P_{k}^{B}-T^{k}\left(\sum_{C} P_{C}^{B} P_{C}^{m+1}\right) \\
& =P_{k}^{B} .
\end{aligned}
$$

Além disso,

$$
d f^{m+1}\left(e_{k}\right)=\eta\left(e_{k}\right)=T^{k}=P_{k}^{m+1} .
$$

Isto implica que $d f\left(e_{k}\right)$ é a k-ésima coluna de $P$. Como $P$ é invertível, $d f$ tem posto máximo, logo $f$ é imersão. Agora, como $P \in S O^{+}\left(\mathbb{E}^{m+2}\right)$, temos que

$$
\left\langle d f\left(e_{p}\right), d f\left(e_{q}\right)\right\rangle_{\varepsilon}=\sum_{C} P_{p}^{C} P_{q}^{C}=\delta_{p q},
$$

$\operatorname{logo} f$ é uma isometria. Agora, podemos diminuir $U_{1}$ de maneira que $f$ seja um mergulho. Em particular, $f$ é injetora.

Observe que a coluna 0 de $P(x)$ é $\left(f^{0}(x), \ldots, f^{m}(x), 0\right)$. Como $\left(f^{0}, \ldots, f^{m}\right) \in \mathbb{Q}_{\varepsilon}^{m}$, a coluna 0 de $P(x)$ é o vetor unitário $\bar{N}(f(x))$ a $\mathbb{Q}_{\varepsilon}^{m} \times \mathbb{R}$ em $\mathbb{E}^{m+2}$ no ponto $f(x)$. As colunas de 1 a $n$ de $P$ são $\left(d f\left(e_{1}\right), \ldots, d f\left(e_{n}\right)\right)$, que formam um referencial ortonormal em $f\left(U_{1}\right)$ para $\mathbb{Q}_{\varepsilon}^{m} \times \mathbb{R}$ no ponto $f(x)$. Agora note que as colunas restantes de $P$, isto é, as colunas de $n+1$ a $m+1$, formam um referencial ortonormal de $f\left(U_{1}\right)$ em $\mathbb{Q}_{\varepsilon}^{m} \times \mathbb{R}$ no ponto $f(x)$, e denotaremos este referencial por $\left\{N_{\alpha}(f(x))\right\}_{\alpha=n+1}^{m+1}$. Vamos denotar a B-ésima coluna de $P$ por $P_{B}$. Escrevendo $X_{j}=d f\left(e_{j}\right)$ e denotando por $\tilde{A}_{\alpha}$ o operador forma de $f\left(U_{1}\right)$ com respeito à $N_{\alpha}$ associado à $f$, temos que

$$
\begin{aligned}
\left\langle\tilde{A}_{\alpha}\left(X_{k}\right), X_{j}\right\rangle_{\varepsilon} & =\left\langle\left(\bar{\nabla}_{X_{k}} X_{j}\right)^{\perp}, N_{\alpha}\right\rangle_{\varepsilon}=\left\langle\bar{\nabla}_{X_{k}} X_{j}, N_{\alpha}\right\rangle_{\varepsilon}=\left\langle\left(\bar{\nabla}_{X_{k}} X_{j}\right)^{\top}, N_{\alpha}\right\rangle_{\varepsilon} \\
& =\left\langle\bar{\nabla}_{X_{k}} X_{j}, N_{\alpha}\right\rangle_{\varepsilon}=\left\langle d X_{j}\left(X_{k}\right), N_{\alpha}\right\rangle_{\varepsilon}=\varepsilon d P_{j}^{0}\left(e_{k}\right) P_{\alpha}^{0}+\sum_{C=1}^{m+1} d P_{j}^{C}\left(e_{k}\right) P_{\alpha}^{C} \\
& =\varepsilon \sum_{B} P_{B}^{0} \omega_{j}^{B}\left(e_{k}\right) P_{\alpha}^{0}+\sum_{B} \sum_{C=1}^{m+1} P_{B}^{C} \omega_{j}^{B}\left(e_{k}\right) P_{\alpha}^{C} \\
& =\sum_{B} \omega_{j}^{B}\left(e_{k}\right)\left(\varepsilon P_{B}^{0} P_{\alpha}^{0}+\sum_{C=1}^{m+1} P_{B}^{C} P_{\alpha}^{C}\right)
\end{aligned}
$$


Como $P \in S O^{+}\left(\mathbb{E}^{m+2}\right)$,

$$
\varepsilon P_{B}^{0} P_{\alpha}^{0}+\sum_{C=1}^{m+1} P_{B}^{C} P_{\alpha}^{C}= \begin{cases}0 & \text { if } B \neq \alpha, \\ 1 & \text { if } B=\alpha .\end{cases}
$$

Logo $\left\langle\tilde{A}_{\alpha}\left(X_{k}\right), X_{j}\right\rangle_{\varepsilon}=\omega_{j}^{\alpha}\left(e_{k}\right)=\left\langle A_{\alpha}\left(e_{k}\right), e_{j}\right\rangle$. Por outro lado,

$$
\begin{aligned}
\left\langle\tilde{A}_{\alpha}\left(X_{k}\right), X_{j}\right\rangle_{\varepsilon} & =\left\langle\tilde{A}_{\alpha} \circ d f\left(e_{k}\right), d f\left(e_{j}\right)\right\rangle_{\varepsilon} \\
& =\left\langle d f\left(d f^{-1} \circ \tilde{A}_{\alpha} \circ d f\left(e_{k}\right)\right), d f\left(e_{j}\right)\right\rangle_{\varepsilon} \\
& =\left\langle d f^{-1} \circ \tilde{A}_{\alpha} \circ d f\left(e_{k}\right), e_{j}\right\rangle .
\end{aligned}
$$

Portanto $\left\langle A_{\alpha}\left(e_{k}\right), e_{j}\right\rangle=\left\langle d f^{-1} \circ \tilde{A}_{\alpha} \circ d f\left(e_{k}\right), e_{j}\right\rangle$, e concluímos que

$$
\tilde{A}_{\alpha}=d f \circ A_{\alpha} \circ d f^{-1} .
$$

Denote as formas de conexão em $T^{\perp}\left(f\left(U_{1}\right)\right)$ por $\tilde{\omega}_{\alpha}^{\beta}$. Então

$$
\begin{aligned}
\tilde{\omega}_{\alpha}^{\beta}\left(d f\left(e_{k}\right)\right) & =\tilde{\omega}_{\alpha}^{\beta}\left(X_{k}\right)=\left\langle\left(\bar{\nabla}_{X_{k}} N_{\alpha}\right)^{\perp}, N_{\beta}\right\rangle_{\varepsilon}=\left\langle\bar{\nabla}_{X_{k}} N_{\alpha}, N_{\beta}\right\rangle_{\varepsilon} \\
& =\left\langle\left(\overline{\bar{\nabla}}_{X_{k}} N_{\alpha}\right)^{\top}, N_{\beta}\right\rangle_{\varepsilon}=\left\langle\overline{\bar{\nabla}}_{X_{k}} N_{\alpha}, N_{\beta}\right\rangle_{\varepsilon}=\left\langle d N_{\alpha}\left(X_{k}\right), N_{\beta}\right\rangle_{\varepsilon} \\
& =\varepsilon d P_{\alpha}^{0}\left(e_{k}\right) P_{\beta}^{0}+\sum_{C=1}^{m+1} d P_{\alpha}^{C}\left(e_{k}\right) P_{\beta}^{C} \\
& =\varepsilon \sum_{B} P_{B}^{0} \omega_{\alpha}^{B}\left(e_{k}\right) P_{\beta}^{0}+\sum_{B} \sum_{C=1}^{m+1} P_{B}^{C} \omega_{\alpha}^{B}\left(e_{k}\right) P_{\beta}^{C} \\
& =\sum_{B} \omega_{\alpha}^{B}\left(e_{k}\right)\left(\varepsilon P_{B}^{0} P_{\beta}^{0}+\sum_{C=1}^{m+1} P_{B}^{C} P_{\beta}^{C}\right) .
\end{aligned}
$$

Mas

$$
\varepsilon P_{B}^{0} P_{\beta}^{0}+\sum_{C=1}^{m+1} P_{B}^{C} P_{\beta}^{C}= \begin{cases}0 & \text { if } B \neq \beta, \\ 1 & \text { if } B=\beta .\end{cases}
$$

Portanto $\tilde{\omega}_{\alpha}^{\beta}\left(d f\left(e_{k}\right)\right)=\omega_{\alpha}^{\beta}\left(e_{k}\right)$, e concluímos que

$$
f^{*} \tilde{\omega}_{\alpha}^{\beta}=\omega_{\alpha}^{\beta}
$$

Considere agora a representação de $\partial / \partial t=E_{m+1}$ no referencial

$$
\left(\bar{N}, X_{1}, \ldots, X_{n}, N_{n+1}, \ldots, N_{m+1}\right) .
$$

Pela proposição (2.2.7), a matriz $P$ é única, logo, pelo que fizemos na seção 1.3 , as colunas de $P$ são as coordenadas de $\bar{N}, X_{1}, \ldots, X_{n}, N_{n+1}, \ldots, N_{m+1}$ no referencial $\left\{E_{C}\right\}_{C=0}^{m+1}$, e portanto

$$
\begin{gathered}
\bar{N}=\sum_{C} P_{0}^{C} E_{C} \Rightarrow\left\langle\bar{N}, \frac{\partial}{\partial t}\right\rangle_{\varepsilon}=P_{0}^{m+1}=T^{0}=0, \\
X_{j}=\sum_{C} P_{j}^{C} E_{C} \Rightarrow\left\langle X_{j}, \frac{\partial}{\partial t}\right\rangle_{\varepsilon}=P_{j}^{m+1}=T^{j}, \\
N_{\alpha}=\sum_{C} P_{\alpha}^{C} E_{C} \Rightarrow\left\langle N_{\alpha}, \frac{\partial}{\partial t}\right\rangle_{\varepsilon}=P_{\alpha}^{m+1}=\nu^{\alpha},
\end{gathered}
$$


e portanto

$$
\frac{\partial}{\partial t}=\sum_{j} T^{j} X_{j}+\sum_{\alpha} \nu^{\alpha} N_{\alpha}=d f(T)+\sum_{\alpha} \nu^{\alpha} N_{\alpha} .
$$

Provaremos agora que a imersão é única a menos de uma isometria global de $\mathbb{Q}_{\varepsilon}^{m} \times \mathbb{R}$. Seja $\tilde{f}: U_{2} \rightarrow \mathbb{Q}_{\varepsilon}^{m} \times \mathbb{R}$ outra imersão satisfazendo a conclusão do teorema, onde $U_{2}$ é uma vizinhança de $x_{0}$ simplesmente conexa contida em $U_{1}$. Seja $\left\{\tilde{X}_{C}\right\}_{C=0}^{m+1}$ o referencial associado, ou seja, $\tilde{X}_{j}=d \tilde{f}\left(e_{j}\right)$, $\tilde{X}_{\alpha}$ normais à $\tilde{f}\left(U_{1}\right)$ em $\mathbb{Q}_{\varepsilon}^{m} \times \mathbb{R}$ e $\tilde{X}_{0}$ normal à $\mathbb{Q}_{\varepsilon}^{m} \times \mathbb{R}$ em $\mathbb{E}^{m+2}$, e seja $\tilde{P}$ a matriz cujas colunas são as coordenadas de $\tilde{X}_{C}$ na base $\left\{E_{C}\right\}$. Pela seção 1.3 , segue que $d \tilde{P}^{-1} d \tilde{P}=\Omega$, e como $\tilde{f}$ satisfaz a conclusão do teorema, por (4) segue que a última linha de $\tilde{P}$ é $\left(T^{0}, \ldots T^{m+1}\right)$, ou seja, $\tilde{P}(x) \in \mathcal{Z}(x)$. Pela demonstração da proposição (2.2.7), existe uma única matriz $B \in S O^{+}\left(\mathbb{E}^{m+1}\right)$ tal que

$$
P\left(x_{0}\right)=\left(\begin{array}{cc}
B & 0 \\
0 & 1
\end{array}\right) \tilde{P}\left(x_{0}\right)
$$

pois $P\left(x_{0}\right), \tilde{P}\left(x_{0}\right) \in \mathcal{Z}\left(x_{0}\right)$. Seja $S: \mathbb{E}^{m+2} \rightarrow \mathbb{E}^{m+2}$ a transformação linear tal que, na base $\left\{E_{C}\right\}$, a matriz associada a $S$ é

$$
[S]=\left(\begin{array}{cc}
B & 0 \\
0 & 1
\end{array}\right) \text {. }
$$

Claramente temos que $[S] \in S O^{+}\left(\mathbb{E}^{m+2}\right)$, logo $S$ é uma isometria em $\mathbb{E}^{m+2}$. Agora, como $B \in$ $S O^{+}\left(\mathbb{E}^{m+1}\right)$, segue que a restrição de $S$ em $\mathbb{Q}_{\varepsilon}^{m} \times \mathbb{R}$ é uma isometria de $\mathbb{Q}_{\varepsilon}^{m} \times \mathbb{R}$. Observe que como $S$ é linear, $[d S]=[S]$. Seja agora $R: \mathbb{E}^{m+2} \rightarrow \mathbb{E}^{m+2}$ dada por $R(x)=x+\left(0, \ldots, 0, f^{m+1}\left(x_{0}\right)-\tilde{f}^{m+1}\left(x_{0}\right)\right)$. Note que $d R=I d$, onde $I d$ denota a aplicação identidade. Assim $R$ é uma isometria em $\mathbb{Q}_{\varepsilon}^{m} \times \mathbb{R}$, e portanto podemos definir a isometria $L: \mathbb{Q}_{\varepsilon}^{m} \times \mathbb{R} \rightarrow \mathbb{Q}_{\varepsilon}^{m} \times \mathbb{R}$ dada por $L(x)=R(S(x))$. Observe que $d L=I d \circ d S=d S$.

Como $P\left(x_{0}\right)=[S] \tilde{P}\left(x_{0}\right)=[d L] \tilde{P}\left(x_{0}\right)$, temos que $L$ preserva as orientações de $\mathbb{Q}_{\varepsilon}^{m}$ e de $\mathbb{R}$ e

$$
\begin{gathered}
\bar{N}\left(f\left(x_{0}\right)\right)=d L_{f\left(x_{0}\right)} \tilde{X}_{0}\left(f\left(x_{0}\right)\right), \\
X_{i}\left(f\left(x_{0}\right)\right)=d L_{f\left(x_{0}\right)} \tilde{X}_{i}\left(f\left(x_{0}\right)\right), \\
N_{\alpha}\left(f\left(x_{0}\right)\right)=d L_{f\left(x_{0}\right)} \tilde{X}_{\alpha}\left(f\left(x_{0}\right)\right) .
\end{gathered}
$$

Além disso, como $\bar{N}\left(f\left(x_{0}\right)\right)=\left(f^{0}\left(x_{0}\right), \ldots, f^{m}\left(x_{0}\right), 0\right)$ e $\tilde{X}_{0}\left(f\left(x_{0}\right)\right)=\left(\tilde{f}^{0}\left(x_{0}\right), \ldots, \tilde{f}^{m}\left(x_{0}\right), 0\right)$, segue que $f\left(x_{0}\right)=L\left(\tilde{f}\left(x_{0}\right)\right)$. Portanto, a menos de uma isometria em $\mathbb{Q}_{\varepsilon}^{m} \times \mathbb{R}$, podemos assumir que $f\left(x_{0}\right)=\tilde{f}\left(x_{0}\right), \bar{N}\left(f\left(x_{0}\right)\right)=\tilde{X}_{0}\left(f\left(x_{0}\right)\right), X_{i}\left(f\left(x_{0}\right)\right)=\tilde{X}_{i}\left(f\left(x_{0}\right)\right)$ e $N_{\alpha}\left(f\left(x_{0}\right)\right)=\tilde{X}_{\alpha}\left(f\left(x_{0}\right)\right)$, ou seja, $P\left(x_{0}\right)=\tilde{P}\left(x_{0}\right)$.

As matrizes $P$ e $\tilde{P}$ satisfazem as equações $P^{-1} d P=\Omega$ e $\tilde{P}^{-1} d \tilde{P}=\Omega, P(x), \tilde{P}(x) \in \mathcal{Z}(x)$ e $P\left(x_{0}\right)=\tilde{P}\left(x_{0}\right)$. Pela unicidade de soluções da equação na proposição $(2.2 .7)$, temos que $P(x)=$ $\tilde{P}(x)$, para todo $x \in U_{1}$. Considerando a coluna 0 dessas matrizes, temos que $f^{0}=\tilde{f}^{0}, \ldots, f^{m}=\tilde{f}^{m}$. Como $d \tilde{f}^{m+1}\left(e_{j}\right)=\left\langle\partial / \partial t, d \tilde{f}\left(e_{j}\right)\right\rangle=T^{j}=\left\langle T, e_{j}\right\rangle=\eta\left(e_{j}\right)$, temos $d \tilde{f}^{m+1}=\eta=d f^{m+1}$. Mas $f^{m+1}\left(x_{0}\right)=\tilde{f}^{m+1}\left(x_{0}\right)$, logo, pela unicidade, temos $f^{m+1}=\tilde{f}^{m+1}$ e concluímos que $f=\tilde{f}$ em $U_{2}$.

Falta mostrar que esta imersão $f$ pode ser estendida a $M$ de maneira única. Seja $x_{1} \in M$. Como $M$ é simplesmente conexo, existe uma curva $\Gamma:[0,1] \rightarrow M$ tal que $\Gamma(0)=x_{0}$ e $\Gamma(1)=x_{1}$. Pelo que vimos até agora, para cada ponto $p \in \Gamma$, existem uma vizinhança $W_{p} \operatorname{com} p \in W_{p}$ e uma imersão isométrica de $W_{p}$ em $\mathbb{Q}_{\varepsilon}^{m} \times \mathbb{R}$ que satisfaz as propriedades do teorema. Logo $\Gamma \subset \cup_{p \in \Gamma} W_{p}$. Como $\Gamma$ é compacta, obtemos uma subcobertura finita $W_{1}, W_{2}, \ldots W_{r}$ de $\Gamma$, e podemos supor que $W_{1}=U_{1}$. Seja $f_{l}$ a imersão isométrica de $W_{l}$ em $\mathbb{Q}_{\varepsilon}^{m} \times \mathbb{R}$ com as propriedades do teorema, com $f_{1}=f$. Se $W_{1} \cap W_{l} \neq \emptyset$, compondo $f_{l}$ com uma isometria se nessessário, temos $f_{1}=f_{l}$ em $W_{1} \cap W_{l}$, logo podemos estender $f$ a $W_{1} \cup W_{l}$ de maneira única. Aplicando o mesmo método sucessivamente, podemos estender $f$ para cada $W_{k}$ de maneira única. Em particular, $f\left(x_{1}\right)$ está definido. Como $M$ é simplesmente conexa, o valor de $f\left(x_{1}\right)$ não depende da escolha da curva $\Gamma$. Concluímos que podemos estender $f$ para toda a variedade $M$. 
Proposição 2.2.9. Seja $f: M \rightarrow \mathbb{Q}_{\varepsilon}^{n} \times \mathbb{R}$ uma imersão de uma variedade Riemanniana $M$ de dimensão $n$ em $\mathbb{Q}_{\varepsilon}^{n} \times \mathbb{R}$ e $(\langle\rangle, A, T,, \nu)$ os dados compatíveis determinados por $f$. Então $(\langle\rangle,-A, T,,-\nu)$, $(\langle\rangle,-A,-T,, \nu)$ e $(\langle\rangle, A,-T,,-\nu)$ também satisfazem as equações de compatibilidade e correspondem a imersão $\sigma \circ f$, onde $\sigma$ é uma isometria de $\mathbb{Q}_{\varepsilon}^{n} \times \mathbb{R}$ :

1. que troca a orientação de $\mathbb{Q}_{\varepsilon}^{n}$ e preserva a orientação de $\mathbb{R}$ no caso de $(\langle\rangle,-A, T,,-\nu)$,

2. que preserva a orientação de $\mathbb{Q}_{\varepsilon}^{n}$ e troca orientação de $\mathbb{R}$ no caso de $(\langle\rangle,-A,-T,, \nu)$,

3. que troca ambas as orientações de $\mathbb{Q}_{\varepsilon}^{n}$ e de $\mathbb{R}$ no caso de $(\langle\rangle, A,-T,,-\nu)$.

Demonstração. Vamos provar apenas o item 1. Os demais itens são análogos. Seja $\tilde{f}=\sigma \circ f$. Então o normal a $\mathbb{Q}_{\varepsilon}^{n} \times \mathbb{R}$ em $\mathbb{E}^{n+2}$ é $\sigma \circ \bar{N}$, e como $\sigma$ troca a orientação de $\mathbb{Q}_{\varepsilon}^{n} \times \mathbb{R}$, o normal a $\tilde{f}(M)$ em $\mathbb{Q}_{\varepsilon}^{n} \times \mathbb{R}$ é $\tilde{N}=-\sigma \circ N$. Pelo que vimos na demonstração do teorema 2.2 .8 , temos $\tilde{A}=-A$. Além disso temos $\frac{\partial}{\partial t}=d f(T)+\nu N$, logo, como $\sigma$ preserva a orientação de $\mathbb{R}$, temos

$$
\frac{\partial}{\partial t}=\sigma \circ \frac{\partial}{\partial t}=\sigma \circ d f(t)+\nu \sigma \circ N=d \tilde{f}(T)-\nu \tilde{N}
$$

e concluímos que $\tilde{T}=T$ e $\tilde{\nu}=-\nu$. 


\section{Capítulo 3}

\section{Imersões Mínimas}

Neste capítulo vamos aplicar o Teorema Fundamental das Imersões no estudo de superfícies mínimas de $\mathbb{Q}_{\varepsilon}^{2} \times \mathbb{R}$. Como trabalharemos com superfícies com codimensão 1, podemos reescrever o teorema 2.2 .8 , juntamente com as equações de compatibilidade para superfícies em $\mathbb{Q}_{\varepsilon}^{2} \times \mathbb{R}$, da seguinte maneira:

Teorema 3.0.10. Seja $\Sigma$ uma variedade Riemanniana simplesmente conexa de dimensão 2, com métrica $\langle$,$\rangle , conexão \nabla$ e tensor curvatura $R$ e $K$ a curvatura de $\langle$,$\rangle . Sejam A: T \Sigma \rightarrow T \Sigma$ tensor simétrico em $\Sigma, \nu$ uma função diferenciável em $\Sigma$ e $T$ um campo de vetores em $\Sigma$ tais que $\|T\|^{2}+\nu^{2}=1$. Denote por $K$ a curvatura de $\langle$,$\rangle . Suponha que (\langle\rangle, A, T,, \nu)$ satisfaz as equações de compatibilidade para superfícies em $\mathbb{Q}_{\varepsilon}^{2} \times \mathbb{R}$ :

$$
\begin{gathered}
K=\operatorname{det} A+\varepsilon \nu^{2} \\
\nabla_{X} A(Y)-\nabla_{Y} A(X)-A([X, Y])=\varepsilon \nu(\langle Y, T\rangle X-\langle X, T\rangle Y), \\
\nabla_{X} T=\nu A(X), \\
d \nu(X)+\langle A(X), T\rangle=0 .
\end{gathered}
$$

Então existe uma imersão

$$
f: M \rightarrow \mathbb{Q}_{\varepsilon}^{2} \times \mathbb{R}
$$

tal que o operador forma com respeito ao campo normal unitário $N$ associado a $f$ é

$$
\tilde{A}=d f \circ A \circ d f^{-1}
$$

e o vetor vertical $\frac{\partial}{\partial t}$ associado a $\mathbb{R}$ satisfaz

$$
\frac{\partial}{\partial t}=d f(T)+\nu N
$$

Além disso, a imersão é única a menos de uma isometria global de $\mathbb{Q}_{\varepsilon}^{2} \times \mathbb{R}$ que preserva as orientações de $\mathbb{Q}_{\varepsilon}^{2}$ e $\mathbb{R}$.

Na seção 3.1, utilizando o Teorema Fundamental, provaremos a existência de uma família a 1 parâmetro de deformações isométricas mínimas de uma dada imersão mínima $f: \Sigma \rightarrow \mathbb{Q}_{\varepsilon}^{2} \times \mathbb{R}$ de uma superfície Riemanniana $\Sigma$ em $\mathbb{Q}_{\varepsilon}^{2} \times \mathbb{R}$. Tal família, chamada de família associada, é obtida rotacionando o operador forma e o campo vetorial $T$ em $\Sigma$. Além disso, no caso de superfícies mínimas, reduzimos as equações de compatibilidade a um sistema de duas equações diferenciais parciais envolvendo apenas a função $\nu$, relacionando o problema de encontrar superfícies mínimas em $\mathbb{Q}_{\varepsilon}^{2} \times \mathbb{R}$ ao de encontrar soluções para este sistema.

Feito isso, na seção 3.2 mostraremos como calcular o vetor normal de um superfície parametrizada em $\mathbb{Q}_{\varepsilon}^{2} \times \mathbb{R}$ e seu operador forma, a fim de, nas seções 3.3 e 3.4, dar exemplos de superfícies conjugadas em $\mathbb{S}^{2} \times \mathbb{R}$ e em $\mathbb{H}^{2} \times \mathbb{R}$, respectivamente. Terminaremos este trabalho mostrando que, 
diferentemente do que acontece em $\mathbb{R}^{3}$, existem exemplos de superfícies que possuem duas imersões isométricas mínimas em $\mathbb{H}^{2} \times \mathbb{R}$ que não pertencem a mesma família associada.

\subsection{A Família Associada}

Seja $\Sigma$ uma superfície Riemanniana com métrica $\langle$,$\rangle , conexão Riemanniana \nabla$ e tensor curvatura $R$. Seja $J: T \Sigma \rightarrow T \Sigma$ tal que para todo $p \in \Sigma, J_{p}$ é a rotação de $\frac{\pi}{2}$ no sentido anti-horário em $T_{p} \Sigma$. Sejam também $A: T \Sigma \longrightarrow T \Sigma$ um tensor simétrico em $\Sigma, T$ um campo de vetores em $\Sigma$ e $\nu$ uma função diferenciável em $\Sigma$ tais que $\|T\|^{2}+\nu^{2}=1$. A partir de agora, chamaremos a função $\nu$ de função ângulo.

Proposição 3.1.1. Assuma que $A$ tem traço zero e que $(\langle\rangle, A, T,, \nu)$ satisfaz as equações de compatibilidade para superfícies em $\mathbb{Q}_{\varepsilon}^{2} \times \mathbb{R}$. Para $\theta \in \mathbb{R}$, definimos:

$$
\begin{aligned}
& A_{\theta}=e^{\theta J} A=\cos \theta A+\operatorname{sen} \theta J A, \\
& T_{\theta}=e^{\theta J} T=\cos \theta T+\operatorname{sen} \theta J T,
\end{aligned}
$$

ou seja, $A_{\theta}$ e $T_{\theta}$ são obtidos rotacionando $A$ e $T$ por um ângulo $\theta$. Então $A_{\theta}$ é simétrico e tem traço zero, $\left\|T_{\theta}\right\|^{2}+\nu^{2}=1$ e $\left(\langle\rangle,, A_{\theta}, T_{\theta}, \nu\right)$ satisfaz as equações de compatibilidade para superfícies em $\mathbb{Q}_{\varepsilon}^{2} \times \mathbb{R}$.

Demonstração. Seja $y \in \Sigma$. Tome $e_{1} \in T_{y} \Sigma$ campo de vetores unitário em uma vizinhança de $y$. Então $\left\{e_{1}, J e_{1}\right\}$ é um referencial ortonormal local para $\Sigma$. Observe que como $A$ tem traço zero, $\left\langle A\left(e_{1}\right), e_{1}\right\rangle=-\left\langle A\left(J e_{1}\right), J e_{1}\right\rangle$. Seja $X=x_{1} e_{1}+x_{2} J e_{1} \in \Gamma(T \Sigma)$. Então

$$
\begin{aligned}
\left\langle J A(X), e_{1}\right\rangle & =\left\langle J A\left(x_{1} e_{1}+x_{2} J e_{1}\right), e_{1}\right\rangle \\
& =\left\langle-A\left(x_{1} e_{1}+x_{2} J e_{1}\right), J e_{1}\right\rangle \\
& =-x_{1}\left\langle A\left(e_{1}\right), J e_{1}\right\rangle-x_{2}\left\langle A\left(J e_{1}\right), J e_{1}\right\rangle \\
& =-x_{1}\left\langle e_{1}, A\left(J e_{1}\right)\right\rangle+x_{2}\left\langle A\left(e_{1}\right), e_{1}\right\rangle \\
& =\left\langle e_{1}, A\left(-x_{1} J e_{1}+x_{2} e_{1}\right)\right\rangle \\
& =\left\langle e_{1},-A(J X)\right\rangle
\end{aligned}
$$

$\mathrm{e}$

$$
\begin{aligned}
\left\langle J A(X), J e_{1}\right\rangle & =\left\langle A(X), e_{1}\right\rangle \\
& =x_{1}\left\langle A\left(e_{1}\right), e_{1}\right\rangle+x_{2}\left\langle A\left(J e_{1}\right), e_{1}\right\rangle \\
& =-x_{1}\left\langle A\left(J e_{1}\right), J e_{1}\right\rangle+x_{2}\left\langle J e_{1}, A\left(e_{1}\right)\right\rangle \\
& =\left\langle J e_{1}, A\left(-x_{1} J e_{1}+x_{2} e_{1}\right)\right\rangle \\
& =\left\langle J e_{1},-A(J X)\right\rangle,
\end{aligned}
$$

portanto temos que

$$
\begin{aligned}
J A(X) & =\left\langle J A(X), e_{1}\right\rangle e_{1}+\left\langle J A(X), J e_{1}\right\rangle J e_{1} \\
& =\left\langle e_{1},-A(J X)\right\rangle e_{1}+\left\langle J e_{1},-A(J X)\right\rangle J e_{1} \\
& =-A(J X),
\end{aligned}
$$


ou seja, $J A=-A J$. Seja agora $Y \in \Gamma(T \Sigma)$. Então

$$
\begin{aligned}
\left\langle A_{\theta}(X), Y\right\rangle & =\langle\cos \theta A(X)+\operatorname{sen} \theta J A(X), Y\rangle \\
& =\cos \theta\langle A(X), Y\rangle+\operatorname{sen} \theta\langle J A(X), Y\rangle \\
& =\cos \theta\langle X, A(Y)\rangle+\operatorname{sen} \theta\langle-A(X), J Y\rangle \\
& =\cos \theta\langle X, A(Y)\rangle-\operatorname{sen} \theta\langle X, A(J Y)\rangle \\
& =\cos \theta\langle X, A(Y)\rangle-\operatorname{sen} \theta\langle X,-J A(Y)\rangle \\
& =\langle X,(\cos \theta) A(Y)+(\operatorname{sen} \theta) J A(Y)\rangle \\
& =\left\langle X, A_{\theta}(Y)\right\rangle,
\end{aligned}
$$

portanto $A_{\theta}$ é simétrico. Além disso, se $\operatorname{tr}\left(A_{\theta}\right)$ denota o traço de $A_{\theta}$, temos que

$$
\operatorname{tr}\left(A_{\theta}\right)=\operatorname{tr}(\cos \theta A+\operatorname{sen} \theta J A)=\cos \theta \operatorname{tr}(A)+\operatorname{sen} \theta \operatorname{tr}(J A) .
$$

Como $\operatorname{tr}(A)=0 \mathrm{e}$

$$
\begin{aligned}
\operatorname{tr}(J A) & =\left\langle J A\left(e_{1}\right), e_{1}\right\rangle+\left\langle J A\left(J e_{1}\right), J e_{1}\right\rangle \\
& =\left\langle-A\left(e_{1}\right), J e_{1}\right\rangle+\left\langle A\left(J e_{1}\right), e_{1}\right\rangle \\
& =-\left\langle e_{1}, A\left(J e_{1}\right)\right\rangle+\left\langle A\left(J e_{1}\right), e_{1}\right\rangle \\
& =0
\end{aligned}
$$

segue que $\operatorname{tr}\left(A_{\theta}\right)=0$.

Agora, como $T_{\theta}$ é rotação de $T$, temos que $\left\|T_{\theta}\right\|=\|T\|$, logo $\left\|T_{\theta}\right\|^{2}+\nu^{2}=1$.

Definimos a aplicação $e^{\theta J}: T \Sigma \longrightarrow T \Sigma$ dada por $e^{\theta J}(X)=\cos \theta X+\operatorname{sen} \theta J X$. Note que $e^{\theta J}$ é linear. Temos que

$$
\begin{aligned}
\operatorname{det}\left(e^{\theta J}\right)= & \left\langle e^{\theta J}\left(e_{1}\right), e_{1}\right\rangle\left\langle e^{\theta J}\left(J e_{1}\right), J e_{1}\right\rangle-\left\langle e^{\theta J}\left(e_{1}\right), J e_{1}\right\rangle\left\langle e^{\theta J}\left(J e_{1}\right), e_{1}\right\rangle \\
= & \left\langle\cos \theta e_{1}+\operatorname{sen} \theta J e_{1}, e_{1}\right\rangle\left\langle\cos \theta J e_{1}-\operatorname{sen} \theta e_{1}, J e_{1}\right\rangle \\
& -\left\langle\cos \theta e_{1}+\operatorname{sen} \theta J e_{1}, J e_{1}\right\rangle\left\langle\cos \theta J e_{1}-\operatorname{sen} \theta e_{1}, e_{1}\right\rangle \\
= & \cos ^{2} \theta+\operatorname{sen}^{2} \theta \\
= & 1
\end{aligned}
$$

logo $\operatorname{det}\left(A_{\theta}\right)=\operatorname{det}\left(e^{\theta J} A\right)=\operatorname{det}(A)$. Portanto $\operatorname{det}\left(A_{\theta}\right)+\varepsilon\left(1-\left\|T_{\theta}\right\|^{2}\right)=\operatorname{det}(A)+\varepsilon\left(1-\|T\|^{2}\right)=K$, onde $K$ é a curvatura de $\langle$,$\rangle , ou seja, a equação (3.1) está satisfeita.$

Vamos verificar agora que a aplicação $e^{\theta J}$ comuta com $\nabla_{X}$. Observe que

$$
\nabla_{X} e^{\theta J} Y=\nabla_{X}(\cos \theta Y+\operatorname{sen} \theta J Y)=\cos \theta \nabla_{X} Y+\operatorname{sen} \theta \nabla_{X} J Y .
$$

Como

$$
\begin{aligned}
\nabla_{X} J e_{1} & =\left\langle\nabla_{X} J e_{1}, e_{1}\right\rangle e_{1}+\left\langle\nabla_{X} J e_{1}, J e_{1}\right\rangle J e_{1} \\
& =\left\langle\nabla_{X} J e_{1}, e_{1}\right\rangle e_{1} \\
& =\left\langle-J e_{1}, \nabla_{X} e_{1}\right\rangle e_{1} \\
& =\left\langle e_{1}, J \nabla_{X} e_{1}\right\rangle e_{1} \\
& =\left\langle e_{1}, J \nabla_{X} e_{1}\right\rangle e_{1}+\left\langle\nabla_{X} e_{1}, e_{1}\right\rangle J e_{1} \\
& =\left\langle e_{1}, J \nabla_{X} e_{1}\right\rangle e_{1}+\left\langle J \nabla_{X} e_{1}, J e_{1}\right\rangle J e_{1} \\
& =J \nabla_{X} e_{1},
\end{aligned}
$$


então

$$
\begin{aligned}
\nabla_{X} J Y & =\left\langle\nabla_{X} J Y, e_{1}\right\rangle e_{1}+\left\langle\nabla_{X} J Y, J e_{1}\right\rangle J e_{1} \\
& =\left(X\left\langle J Y, e_{1}\right\rangle-\left\langle J Y, \nabla_{X} e_{1}\right\rangle\right) e_{1}+\left(X\left\langle J Y, J e_{1}\right\rangle-\left\langle J Y, \nabla_{X} J e_{1}\right\rangle\right) J e_{1} \\
& =\left(-X\left\langle Y, J e_{1}\right\rangle+\left\langle Y, J \nabla_{X} e_{1}\right\rangle\right) e_{1}+\left(X\left\langle Y, e_{1}\right\rangle-\left\langle J Y, J \nabla_{X} e_{1}\right\rangle\right) J e_{1} \\
& =\left(-X\left\langle Y, J e_{1}\right\rangle+\left\langle Y, \nabla_{X} J e_{1}\right\rangle\right) e_{1}+\left(X\left\langle Y, e_{1}\right\rangle-\left\langle Y, \nabla_{X} e_{1}\right\rangle\right) J e_{1} \\
& =-\left\langle\nabla_{X} Y, J e_{1}\right\rangle e_{1}+\left\langle\nabla_{X} Y, e_{1}\right\rangle J e_{1} \\
& =\left\langle J \nabla_{X} Y, e_{1}\right\rangle e_{1}+\left\langle J \nabla_{X} Y, J e_{1}\right\rangle J e_{1} \\
& =J \nabla_{X} Y .
\end{aligned}
$$

Desta forma podemos concluir que $\nabla_{X} e^{\theta J} Y=e^{\theta J} \nabla_{X} Y$. Além disso, $e^{\theta J}$ preserva a métrica, pois

$$
\begin{aligned}
\left\langle e^{\theta J} X, e^{\theta J} Y\right\rangle & =\langle\cos \theta X+\operatorname{sen} \theta J X, \cos \theta Y+\operatorname{sen} \theta J Y\rangle \\
& =\cos ^{2} \theta\langle X, Y\rangle+\cos \theta \operatorname{sen} \theta\langle X, J Y\rangle+\cos \theta \operatorname{sen} \theta\langle J X, Y\rangle+\operatorname{sen}^{2} \theta\langle J X, J Y\rangle \\
& =\cos ^{2} \theta\langle X, Y\rangle+\operatorname{sen}^{2} \theta\langle X, Y\rangle \\
& =\langle X, Y\rangle,
\end{aligned}
$$

$\log 0$

$$
\nu A_{\theta}(X)=\nu e^{\theta J} A(X)=e^{\theta J} \nu A(X)=e^{\theta J} \nabla_{X} T=\nabla_{X} e^{\theta J} T=\nabla_{X} T_{\theta}
$$

e

$$
-\left\langle A_{\theta}(X), T_{\theta}\right\rangle=-\left\langle e^{\theta J} A(X), e^{\theta J} T\right\rangle=\langle A(X), T\rangle=d \nu(X) .
$$

Portanto as equações (3.3) e (3.4) estão satisfeitas. Observe agora que

$$
\begin{aligned}
\nabla_{X} e^{\theta J} A(Y)-\nabla_{Y} e^{\theta J} A(X)-e^{\theta J} A([X, Y]) & =e^{\theta J}\left(\nabla_{X} A(Y)-\nabla_{Y} A(X)-A([X, Y])\right) \\
& =\varepsilon \nu e^{\theta J}(\langle T, Y\rangle X-\langle T, X\rangle Y) .
\end{aligned}
$$

Precisamos mostrar que

$$
e^{\theta J}(\langle T, Y\rangle X-\langle T, X\rangle Y)=\left\langle e^{\theta J} T, Y\right\rangle X-\left\langle e^{\theta J} T, X\right\rangle Y .
$$

Nos pontos em que $X=0$, a relação é trivialmente satisfeita. Quando $X \neq 0$, podemos escrever $Y=\lambda X+\mu J X$. Daí

$$
\begin{aligned}
\left\langle e^{\theta J} T, Y\right\rangle X-\left\langle e^{\theta J} T, X\right\rangle Y= & \langle\cos \theta T+\operatorname{sen} \theta J T, \lambda X+\mu J X\rangle X \\
& -\langle\cos \theta T+\operatorname{sen} \theta J T, X\rangle(\lambda X+\mu J X) \\
= & \mu \cos \theta\langle T, J X\rangle X+\mu \operatorname{sen} \theta\langle J T, J X\rangle X \\
& -\mu \cos \theta\langle T, X\rangle J X-\mu \operatorname{sen} \theta\langle J T, X\rangle J X .
\end{aligned}
$$

Por outro lado,

$$
\begin{aligned}
e^{\theta J}(\langle T, Y\rangle X-\langle T, X\rangle Y)= & \langle T, \lambda X+\mu J X\rangle(\cos \theta X+\operatorname{sen} \theta J X) \\
& -\langle T, X\rangle(\lambda \cos \theta X+\lambda \operatorname{sen} \theta J X+\mu \cos \theta J X-\mu \operatorname{sen} \theta X) \\
= & \mu \cos \theta\langle T, J X\rangle X+\mu \operatorname{sen} \theta\langle J T, J X\rangle X \\
& -\mu \cos \theta\langle T, X\rangle J X-\mu \operatorname{sen} \theta\langle J T, X\rangle J X .
\end{aligned}
$$

Comparando as duas expressões, podemos concluir que a equação (3.2) está satisfeita. Portanto $\left(\langle\rangle,, A_{\theta}, T_{\theta}, \nu\right)$ satisfaz as equações de compatibilidade para superfícies em $\mathbb{Q}_{\varepsilon}^{2} \times \mathbb{R}$.

Definição 3.1.2. Uma imersão $f: \Sigma \rightarrow \mathbb{Q}_{\varepsilon}^{2} \times \mathbb{R}$ é chamada de conforme se para todo $p \in \Sigma$ e 
quaisquer $\xi_{1}, \xi_{2} \in T_{p} \Sigma$ temos

$$
\left\langle d f_{p}\left(\xi_{1}\right), d f_{p}\left(\xi_{2}\right)\right\rangle_{\varepsilon}=\lambda(p)^{2}\left\langle\xi_{1}, \xi_{2}\right\rangle,
$$

onde $\lambda$ é uma função diferenciável em $\Sigma$ que nunca se anula.

Utilizando a proposição 3.1.1 e o Teorema Fundamental 2.2.8, mostraremos agora que dada uma imersão isométrica mínima conforme de uma superfície Riemanniana em $\mathbb{Q}_{\varepsilon}^{2} \times \mathbb{R}$, é possível construir uma família a um parâmetro de imersões isometricas mínimas conformes da mesma superfície em $\mathbb{Q}_{\varepsilon}^{2} \times \mathbb{R}$

Teorema 3.1.3. Sejam $\Sigma$ uma superfície Riemanniana simplesmente conexa e $f: \Sigma \longrightarrow \mathbb{Q}_{\varepsilon}^{2} \times \mathbb{R}$ uma imersão mínima conforme. Sejam $N$ o normal induzido por $f$ e $A$ o operador simétrico induzido pelo operador forma $\tilde{A}$ em $f(\Sigma)$, isto é, $A=d f^{-1} \circ \tilde{A} \circ d f$. Sejam ainda $T$ o campo vetorial em $\Sigma$ tal que $d f(T)$ é a projeção de $\frac{\partial}{\partial t}$ em $T f(\Sigma)$ e $\nu=\left\langle N, \frac{\partial}{\partial t}\right\rangle$.

Seja $z_{0} \in \Sigma$. Então existe uma única família $\left(f_{\theta}\right)_{\theta \in \mathbb{R}}$ de imersões mínimas conformes $f_{\theta}: \Sigma \longrightarrow$ $\mathbb{Q}_{\varepsilon}^{2} \times \mathbb{R}$ tais que:

1. $f_{\theta}\left(z_{0}\right)=f\left(z_{0}\right)$ e $\left(d f_{\theta}\right)_{z_{0}}=(d f)_{z_{0}}$

2. As métricas induzidas em $\Sigma$ por $f$ e $f_{\theta}$ são as mesmas;

3. O operador simétrico em $\Sigma$ induzido pelo operador forma de $f_{\theta}(\Sigma)$ é $e^{\theta J} A$;

4. $\frac{\partial}{\partial t}=d f_{\theta}\left(e^{\theta J} T\right)+\nu N^{\theta}$, onde $N^{\theta}$ é o normal unitário a $f_{\theta}$. Além disso temos $f_{0}=f$, e a família $f_{\theta}$ é contínua com relação a $\theta$.

Demonstração. Seja $\langle$,$\rangle a métrica em \Sigma$ induzida por $f$. Então $(\langle\rangle, A, T,, \nu)$ satisfaz as equações de compatibilidade para $\mathbb{Q}_{\varepsilon}^{2} \times \mathbb{R}$. Pela proposição 3.1.1, $\left(\langle\rangle,, A_{\theta}, T_{\theta}, \nu\right)$ também satisfaz as equações de compatibilidade para $\mathbb{Q}_{\varepsilon}^{2} \times \mathbb{R}$. Portanto, pelo teorema 2.2 .8 , para cada $\theta \in \mathbb{R}$ existe uma única imersão $f_{\theta}$ (a menos de isometria de $\mathbb{Q}_{\varepsilon}^{2} \times \mathbb{R}$ ) satisfazendo as propriedades do teorema. De fato, utilizando uma isometria de $\mathbb{Q}_{\varepsilon}^{2} \times \mathbb{R}$ se necessário, podemos supor que a imersão $f_{\theta}$ satisfaz (1), para todo $\theta \in \mathbb{R}$. Note que (2), (3) e (4) são conclusões diretas do teorema 2.2.8. Além disso, como $f$ é conforme, por (2) temos que $f_{\theta}$ também é conforme.

Provaremos agora que a família $f_{\theta}$ é contínua com relação à $\theta$. Considere um referencial ortonormal $\left\{e_{1}, e_{2}\right\}$ em $\Sigma$, com $e_{2}=J e_{1}$. Seja $\left\{\omega^{1}, \omega^{2}\right\}$ a base dual de $\left\{e_{1}, e_{2}\right\}$ e $\left\{\omega_{j}^{i}\right\}_{i, j=1}^{2}$ as formas de conexão de $\Sigma$. Então $\left(\langle\rangle,, A_{\theta}, T_{\theta}, \nu\right)$ define uma matriz $\Omega_{\theta}$ de 1-formas em $\Sigma$ dadas por

$$
\begin{aligned}
& \left(\Omega_{\theta}\right)_{j}^{i}=\omega_{j}^{i}, \quad\left(\Omega_{\theta}\right)_{i}^{j}=-\left(\Omega_{\theta}\right)_{j}^{i}, \\
& \left(\Omega_{\theta}\right)_{j}^{3}(X)=\left\langle A_{\theta}(X), e_{j}\right\rangle, \quad\left(\Omega_{\theta}\right)_{3}^{j}=-\left(\Omega_{\theta}\right)_{j}^{3}, \\
& \left(\Omega_{\theta}\right)_{j}^{0}(X)=\varepsilon\left(\left\langle T_{\theta}, e_{j}\right\rangle\left\langle T_{\theta}, X\right\rangle-\omega^{1}(X)\right), \quad\left(\Omega_{\theta}\right)_{0}^{j}=-\varepsilon\left(\Omega_{\theta}\right)_{j}^{0}, \\
& \left(\Omega_{\theta}\right)_{3}^{0}(X)=\varepsilon \nu\left\langle T_{\theta}, X\right\rangle, \quad\left(\Omega_{\theta}\right)_{0}^{3}=-\varepsilon\left(\Omega_{\theta}\right)_{3}^{0}, \\
& \left(\Omega_{\theta}\right)_{0}^{0}=\left(\Omega_{\theta}\right)_{3}^{3}=0 .
\end{aligned}
$$

Note então que $\Omega_{\theta}$ é contínua com relação a $\theta$. Pela proposição 2.2 .7 , para cada $\theta \in \mathbb{R}$ existe uma única matriz de funções $P_{\theta}$ satisfazendo $P_{\theta}^{-1} d P_{\theta}=\Omega_{\theta}$, e pela continuidade de $\Omega_{\theta}$ com relação a $\theta$, segue a continuidade de $P_{\theta}$ com relação a $\theta$. Pela demonstração de 2.2 .8 , temos que $f_{\theta}$ é definida em termos de $A_{\theta}$ e de $\eta(X)=\left\langle T_{\theta}, X\right\rangle$, e segue a continuidade de $f_{\theta}$ com relação a $\theta$, como queríamos.

A família de imersões $\left(f_{\theta}\right)_{\theta \in \mathbb{R}}$ dada pelo teorema 3.1 .3 é chamada de família associada da imersão $f$. A imersão $f_{\frac{\pi}{2}}$ é chamada imersão conjugada de $f$ e a imersão $f_{\pi}$ é chamada imersão oposta de $f$.

Vamos agora à seguinte definição. 
Definição 3.1.4. O operador de Jacobi de uma imersão $x: \Sigma \rightarrow \bar{M}$ de uma superfície Riemanniana $\Sigma$ em uma variedade Riemanniana $\bar{M}$ de dimensão 3 é o operador dado por

$$
L=\Delta+\|A\|^{2}+\operatorname{Ric}(N)
$$

onde $\Delta$ é o Laplaciano da métrica induzida em $\Sigma, N$ é o normal unitário a $x(\Sigma), A$ é o operador forma de $x$ e Ric é a curvatura de Ricci em $\bar{M}$.

Mais detalhes sobre este operador e algumas propriedades podem ser encontradas em [Xin03]. A proposição a seguir será utilizada na demonstração do teorema 3.1.7.

Proposição 3.1.5. Sejam $\Sigma$ uma superfície Riemanniana simplesmente conexa e $x: \Sigma \rightarrow \mathbb{Q}_{\varepsilon}^{2} \times \mathbb{R}$ uma imersão mínima. Então o operador de Jacobi induzido por $x$ é dado por

$$
L=\Delta-2 K+\varepsilon\left(1+\nu^{2}\right),
$$

onde $\Delta$ é o Laplaciano da métrica induzida em $\Sigma$ e $K$ é a curvatura intrinseca de $\Sigma$.

Demonstração. Por (3.1) temos que

$$
-2 \operatorname{det} A=-2 K+2 \varepsilon \nu^{2} .
$$

Como $x$ é imersão mínima,

$$
\begin{aligned}
\|A\|^{2} & =4 H^{2}-2 \operatorname{det} A \\
& =-2 K+2 \varepsilon \nu^{2} \\
\Rightarrow L & =\Delta-2 K+2 \varepsilon \nu^{2}+\operatorname{Ric}(N) .
\end{aligned}
$$

Precisamos mostrar que

$$
2 \varepsilon \nu^{2}+\operatorname{Ric}(N)=\varepsilon\left(1+\nu^{2}\right),
$$

ou seja,

$$
\operatorname{Ric}(N)=\varepsilon\left(1-\nu^{2}\right) .
$$

Sejam $p \in \Sigma$ e $E_{1}, E_{2} \in T_{p} \Sigma$ de maneira que $\left(N(p), E_{1}, E_{2}\right)$ seja uma base ortonormal para $T_{x(p)} \mathbb{Q}_{\varepsilon}^{2} \times \mathbb{R}$. Então a curvatura de Ricci em $p$ é dada por (Veja [Pet06])

$$
\operatorname{Ric}(N)=\left\langle\bar{R}\left(N, E_{1}\right) N, E_{1}\right\rangle_{\varepsilon}+\left\langle\bar{R}\left(N, E_{2}\right) N, E_{2}\right\rangle_{\varepsilon}
$$

Por (1.17) temos

$$
\left\langle\bar{R}\left(N, E_{i}\right) N, E_{i}\right\rangle_{\varepsilon}=\varepsilon\left[\left\langle N_{\mathbb{Q}}, N_{\mathbb{Q}}\right\rangle_{\varepsilon}\left\langle E_{i \mathbb{Q}}, E_{i \mathbb{Q}}\right\rangle_{\varepsilon}-\left\langle E_{i \mathbb{Q}}, N_{\mathbb{Q}}\right\rangle_{\varepsilon}^{2}\right]
$$

Se $p \in \Sigma$ é tal que $\nu(p)^{2}=1$, temos $N=\nu \frac{\partial}{\partial t}$, ou seja, $N_{\mathbb{Q}}=0$. Desta maneira, segue que

$$
\left\langle\bar{R}\left(N, E_{i}\right) N, E_{i}\right\rangle_{\varepsilon}=0,
$$

$\operatorname{logo} \operatorname{Ric}(N)=0$. Se $\nu(p)^{2} \neq 1$, tome $E_{1}=\frac{\frac{\partial}{\partial t}-\nu N}{\sqrt{1-\nu^{2}}}$ e $E_{2}=N \times E_{1}$. Temos

1.

$$
\langle N, N\rangle_{\varepsilon}=1 \Rightarrow\left\langle N_{\mathbb{Q}}, N_{\mathbb{Q}}\right\rangle_{\varepsilon}+\left\langle N_{\mathbb{R}}, N_{\mathbb{R}}\right\rangle_{\varepsilon}=1 \Rightarrow\left\langle N_{\mathbb{Q}}, N_{\mathbb{Q}}\right\rangle_{\varepsilon}=1-\nu^{2},
$$

pois $N_{\mathbb{R}}=\nu \frac{\partial}{\partial t}$.

2.

$$
\left\langle E_{1}, N\right\rangle_{\varepsilon}=0 \Rightarrow\left\langle E_{1 \mathbb{Q}}, N_{\mathbb{Q}}\right\rangle_{\varepsilon}+\left\langle E_{1 \mathbb{R}}, N_{\mathbb{R}}\right\rangle_{\varepsilon}=0
$$


Como

$$
\begin{aligned}
\left\langle E_{1 \mathbb{R}}, N_{\mathbb{R}}\right\rangle_{\varepsilon} & =\left\langle\left\langle E_{1 \mathbb{R}}, \frac{\partial}{\partial t}\right\rangle_{\varepsilon} \frac{\partial}{\partial t}, \nu \frac{\partial}{\partial t}\right\rangle_{\varepsilon} \\
& =\nu\left\langle E_{1 \mathbb{R}}, \frac{\partial}{\partial t}\right\rangle_{\varepsilon} \\
& =\nu \sqrt{1-\nu^{2}},
\end{aligned}
$$

$\operatorname{temos}\left\langle E_{i \mathbb{Q}}, N_{\mathbb{Q}}\right\rangle_{\varepsilon}=-\nu \sqrt{1-\nu^{2}}$.

3.

$$
\left\langle E_{1}, E_{1}\right\rangle_{\varepsilon}=1 \Rightarrow\left\langle E_{1 \mathbb{Q}}, E_{1 \mathbb{Q}}\right\rangle_{\varepsilon}+\left\langle E_{1 \mathbb{R}}, E_{1 \mathbb{R}}\right\rangle_{\varepsilon}=1 \Rightarrow\left\langle E_{1 \mathbb{Q}}, E_{1 \mathbb{Q}}\right\rangle_{\varepsilon}=1-\left(1-\nu^{2}\right)=\nu^{2}
$$

4.

$$
\left\langle E_{2}, N\right\rangle_{\varepsilon}=0 \Rightarrow\left\langle E_{2 \mathbb{Q}}, N_{\mathbb{Q}}\right\rangle_{\varepsilon}+\left\langle E_{2 \mathbb{R}}, N_{\mathbb{R}}\right\rangle_{\varepsilon}=0
$$

Como

$$
\begin{aligned}
\left\langle E_{2}, \frac{\partial}{\partial t}\right\rangle_{\varepsilon} & =\left\langle E_{2}, \frac{\partial}{\partial t}\right\rangle_{\varepsilon}-\nu\left\langle E_{2}, N\right\rangle_{\varepsilon} \\
& =\left\langle E_{2}, \sqrt{1-\nu^{2}} E_{1}\right\rangle_{\varepsilon} \\
& =0
\end{aligned}
$$

temos $E_{2 \mathbb{R}}=0, \operatorname{logo}\left\langle E_{2 \mathbb{Q}}, N_{\mathbb{Q}}\right\rangle_{\varepsilon}=0$.

5.

$$
\left\langle E_{2}, E_{2}\right\rangle_{\varepsilon}=1 \Rightarrow\left\langle E_{2 \mathbb{Q}}, E_{2 \mathbb{Q}}\right\rangle_{\varepsilon}+\left\langle E_{2 \mathbb{R}}, E_{2 \mathbb{R}}\right\rangle \varepsilon=1 \Rightarrow\left\langle E_{2 \mathbb{Q}}, E_{2 \mathbb{Q}}\right\rangle_{\varepsilon}=1
$$

Pelos itens acima, substituindo em (3.6), temos que

$$
\left\langle\bar{R}\left(N, E_{1}\right) N, E_{1}\right\rangle_{\varepsilon}=\varepsilon\left[\left(1-\nu^{2}\right) \nu^{2}-\left(-\nu \sqrt{1-\nu^{2}}\right)^{2}\right]=0
$$

e

$$
\left\langle\bar{R}\left(N, E_{2}\right) N, E_{2}\right\rangle_{\varepsilon}=\varepsilon\left(1-\nu^{2}\right) .
$$

Portanto

$$
\operatorname{Ric}(N)=\varepsilon\left(1-\nu^{2}\right)
$$

como queríamos.

Antes de prosseguir com o próximo teorema, vamos recordar o conceito de campo de Killing: $X \in \Gamma(T M)$ é um campo de Killing $\Leftrightarrow\left\langle\nabla_{Y} X, Z\right\rangle+\left\langle\nabla_{Z} X, Y\right\rangle=0$, para todo $Y, Z \in \Gamma(T M)$. Esta equação é chamada de equação de Killing. Para maiores detalhes sobre campos de Killing, veja [dC79].

Lema 3.1.6. Seja $x: \Sigma \rightarrow \bar{M}$ uma imersão mínima de uma superfície Riemanniana $\Sigma$ em uma variedade Riemanniana $\bar{M}$ de dimensão 3 com métrica $\bar{g}$. Seja $N$ o normal unitário a $x(\Sigma)$. Se $X \in \Gamma(T \bar{M})$ é um campo de Killing, então a função $h=\bar{g}(X, N)$ satisfaz $L h=0$, onde $L$ é o operador de Jacobi.

Demonstração. Veja [Xin03]. 
Teorema 3.1.7. Seja $\Sigma$ uma superfície mínima em $\mathbb{Q}_{\varepsilon}^{2} \times \mathbb{R}$. Então a função ângulo $\nu: \Sigma \rightarrow[-1,1]$ satisfaz

$$
\begin{gathered}
\|\nabla \nu\|^{2}=-\left(1-\nu^{2}\right)\left(K-\varepsilon \nu^{2}\right) \\
\Delta \nu-2 K \nu+\varepsilon\left(1+\nu^{2}\right) \nu=0,
\end{gathered}
$$

onde $K$ denota a curvatura intrínseca de $\Sigma$. Reciprocamente, seja $\Sigma$ uma superfície Riemanniana simplesmente conexa, e $\nu: \Sigma \rightarrow(-1,1)$ uma função diferenciável satisfazendo (3.7) e (3.8), onde $K$ é a curvatura de $\Sigma$. Então existe uma imersão isométrica mínima $f: \Sigma \rightarrow \mathbb{Q}_{\varepsilon}^{2} \times \mathbb{R}$ com função ângulo $\nu$. Além disso, se $g: \Sigma \rightarrow \mathbb{Q}_{\varepsilon}^{2} \times \mathbb{R}$ é outra imersão isométrica mínima com função ângulo $\nu$, então $f$ e $g$ são associadas.

Demonstração. Seja $\Sigma$ uma superfície mínima em $\mathbb{Q}_{\varepsilon}^{2} \times \mathbb{R}$. Considerando o recobrimento duplo orientável se necessário (veja [dC79]), podemos assumir que $\Sigma$ está orientada. Sejam $(\langle\rangle, A, T,, \nu)$ os dados compatíveis de $\Sigma$. Pela equação (3.4) e pela simetria de $A$, temos

$$
\begin{aligned}
& d \nu(X)+\langle A(X), T\rangle=0 \\
\Rightarrow \quad & \langle X, A(T)\rangle=-\langle X, \nabla \nu\rangle \\
\Rightarrow \quad & A(T)=-\nabla \nu .
\end{aligned}
$$

Além disso, como $\Sigma$ é mínima, $A$ tem traço nulo, e como vimos no início da demonstração da proposição 3.1.1, se $J$ denota a rotação por $\frac{\pi}{2}$ em $T \Sigma$, então $A J=-J A$.

Nos pontos em que $T \neq 0$, podemos considerar o referencial ortonormal $\left\{\frac{T}{\|T\|}, \frac{J T}{\|T\|}\right\}$. Daí

$$
\begin{aligned}
\operatorname{det} A & =\left\langle A\left(\frac{T}{\|T\|}\right), \frac{T}{\|T\|}\right\rangle\left\langle A\left(\frac{J T}{\|T\|}\right), \frac{J T}{\|T\|}\right\rangle-\left\langle A\left(\frac{J T}{\|T\|}\right), \frac{T}{\|T\|}\right\rangle\left\langle A\left(\frac{T}{\|T\|}\right), \frac{J T}{\|T\|}\right\rangle \\
& =\frac{1}{\|T\|^{4}}\langle A(T), T\rangle\langle A(J T), J T\rangle-\langle A(J T), T\rangle\langle A(T), J T\rangle \\
& =\frac{1}{\|T\|^{4}}\langle-\nabla \nu, T\rangle\langle\nabla \nu, T\rangle-\langle-\nabla \nu, J T\rangle\langle-\nabla \nu, J T\rangle \\
& =-\frac{\|\nabla \nu\|^{2}}{\|T\|^{2}} .
\end{aligned}
$$

Então, pela equação (3.1) e por $\|T\|^{2}+\nu^{2}=1$, temos

$$
\begin{aligned}
& K=\operatorname{det} A+\varepsilon \nu^{2} \\
\Rightarrow & K=-\frac{\|\nabla \nu\|^{2}}{\|T\|^{2}}+\varepsilon \nu^{2} \\
\Rightarrow & \|\nabla \nu\|^{2}=-\|T\|^{2} K+\|T\|^{2} \varepsilon \nu^{2} \\
\Rightarrow & \|\nabla \nu\|^{2}=-K\left(1-\nu^{2}\right)+\left(1-\nu^{2}\right) \varepsilon \nu^{2} \\
\Rightarrow & \|\nabla \nu\|^{2}=-\left(1-\nu^{2}\right)\left(K-\varepsilon \nu^{2}\right),
\end{aligned}
$$

logo temos (3.7). Para os pontos em que $T=0$, ou seja, $\nu=1$, basta observar que por (3.4) temos

$$
d \nu(X)+\langle A(X), T\rangle=0 \Rightarrow d \nu(X)=0 \Rightarrow\langle\nabla \nu, X\rangle=0,
$$

para todo $X \in \Gamma(T \Sigma)$, $\operatorname{logo} \nabla \nu=0$, e portanto (3.7) está trivialmente satisfeita.

Falta verificar que $\nu$ satisfaz (3.8). Temos que $\frac{\partial}{\partial t}$ é um campo de Killing. De fato, como $\frac{\partial}{\partial t}$ é paralelo,

$$
\bar{\nabla}_{Y} \frac{\partial}{\partial t}=\nabla_{Y_{\mathbb{Q}}}^{\mathbb{Q}} 0+\nabla_{\left\langle Y, \frac{\partial}{\partial t}\right\rangle \frac{\partial}{\partial t}}^{\mathbb{R}} \frac{\partial}{\partial t}=0,
$$

e segue que $\frac{\partial}{\partial t}$ satisfaz a equação de Killing. Assim, pelo lema 3.1.6, a função $\nu=\left\langle\frac{\partial}{\partial t}, N\right\rangle_{\varepsilon}$ satisfaz 
$L \nu=0$, onde $L$ é o operador de Jacobi. Pelo lema 3.1.5 segue que $\nu$ satisfaz (3.8). Note que além disso, para os pontos em que $\nu^{2} \neq 1$, ou seja, $T \neq 0$,

$$
\begin{aligned}
\nabla_{X} T & =\nu A(X) \\
& =\nu\left(\left\langle A(X), \frac{T}{\|T\|}\right\rangle \frac{T}{\|T\|}+\left\langle A(X), \frac{J T}{\|T\|}\right\rangle \frac{J T}{\|T\|}\right) \\
& =\frac{\nu}{\|T\|^{2}}(\langle X, A(T)\rangle T+\langle X, A(J T)\rangle J T) \\
& =\frac{\nu}{1-\nu^{2}}(-\langle X, \nabla \nu\rangle T+\langle X, J \nabla \nu\rangle J T) .
\end{aligned}
$$

Seja agora $\Sigma$ uma superfície Riemanniana simplesmente conexa com métrica $\langle$,$\rangle , e seja \nu: \Sigma \rightarrow$ $(-1,1)$ uma função satisfazendo (3.7) e (3.8). Primeiramente vamos encontrar um campo vetorial $T$ satisfazendo $\|T\|^{2}+\nu^{2}=1$ e (3.9). Sejam $\left\{e_{1}, e_{2}\right\}$, com $e_{2}=J e_{1}$, um referencial ortonormal definido num aberto $U \subset \Sigma$, e seja $\left\{\omega^{1}, \omega^{2}\right\}$ seu referencial dual. Podemos supor que $U$ é simplesmente conexo. Temos que $\nabla_{X} e_{1}=\omega_{1}^{2}(X) e_{2}$ e $\nabla_{X} e_{2}=\omega_{2}^{1}(X) e_{1}$, para todo $X \in \Gamma(T \Sigma)$. Sejam $\theta: U \rightarrow \mathbb{R}$ uma função diferenciável e $T=\sqrt{1-\nu^{2}} e^{\theta J} e_{1}$. Como $\nu^{2} \neq 1$, temos que

$$
\begin{aligned}
\nabla_{X} T & =\nabla_{X}\left(\sqrt{1-\nu^{2}} e^{\theta J} e_{1}\right) \\
& =X\left(\sqrt{1-\nu^{2}}\right) e^{\theta J} e_{1}+\sqrt{1-\nu^{2}} \nabla_{X} e^{\theta J} e_{1} \\
& \left.=d\left(\sqrt{1-\nu^{2}}\right)(X) e^{\theta J} e_{1}\right)+\sqrt{1-\nu^{2}} \nabla_{X}\left(\cos \theta e_{1}+\operatorname{sen} \theta e_{2}\right) \\
& =-\frac{\nu}{1-\nu^{2}} d \nu(X) T+\sqrt{1-\nu^{2}} d \theta(X) e^{\theta J} e_{2}+\sqrt{1-\nu^{2}} \omega_{1}^{2}(X) e^{\theta J} e_{2} \\
& =-\frac{\nu}{1-\nu^{2}} d \nu(X) T+d \theta(X) J T+\omega_{1}^{2}(X) J T
\end{aligned}
$$

Portanto, $T$ satisfaz (3.9) se e somente se

$$
\frac{\nu}{1-\nu^{2}}\langle X, J \nabla \nu\rangle=d \theta(X)+\omega_{1}^{2}(X)
$$

para todo $X \in \Gamma(T U)$, ou seja, se e somente se

$$
d \theta=-\frac{\nu}{1-\nu^{2}}(d \nu \circ J)-\omega_{1}^{2}
$$

Vamos mostrar que $-\frac{\nu}{1-\nu^{2}}(d \nu \circ J)-\omega_{1}^{2}$ é uma forma fechada, e portanto existe $\theta$ satisfazendo (3.10) e, consequentemente, existe um campo $T$ satisfazendo (3.9). Temos

$$
d\left(\frac{\nu}{1-\nu^{2}}(d \nu \circ J)+\omega_{1}^{2}\right)=\frac{1+\nu^{2}}{1-\nu^{2}} d \nu \wedge(d \nu \circ J)+\frac{\nu}{1-\nu^{2}} d(d \nu \circ J)+d \omega_{1}^{2} .
$$

Observe que, se $X, Y \in \Gamma(T U)$, escrevendo $X=x_{1} e_{1}+x_{2} e_{2}$ e $Y=y_{1} e_{1}+y_{2} e_{2}$, temos

$$
\begin{aligned}
(d \nu \wedge(d \nu \circ J))(X, Y)= & d \nu(X)(d \nu \circ J)(Y)-d \nu(Y)(d \nu \circ J)(X) \\
= & {\left[x_{1} d \nu\left(e_{1}\right)+x_{2} d \nu\left(e_{2}\right)\right]\left[y_{1} d \nu\left(J e_{1}\right)+y_{2} d \nu\left(J e_{2}\right)\right] } \\
& -\left[y_{1} d \nu\left(e_{1}\right)+y_{2} d \nu\left(e_{2}\right)\right]\left[x_{1} d \nu\left(J e_{1}\right)+x_{2} d \nu\left(J e_{2}\right)\right] \\
= & x_{1} y_{2} d \nu\left(e_{1}\right) d \nu\left(J e_{2}\right)+x_{2} y_{1} d \nu\left(e_{2}\right) d \nu\left(J e_{1}\right) \\
& -y_{1} x_{2} d \nu\left(e_{1}\right) d \nu\left(J e_{2}\right)-y_{2} x_{1} d \nu\left(e_{2}\right) d \nu\left(J e_{1}\right) \\
= & -x_{1} y_{2} d \nu\left(e_{1}\right)^{2}+x_{2} y_{1} d \nu\left(e_{2}\right)^{2}+y_{1} x_{2} d \nu\left(e_{1}\right)^{2}-y_{2} x_{1} d \nu\left(e_{2}\right)^{2} \\
= & -\left(d \nu\left(e_{1}\right)^{2}+d \nu\left(e_{2}\right)^{2}\right) \omega^{1} \wedge \omega^{2}(X, Y) \\
= & -\|\nabla \nu\|^{2} \omega^{1} \wedge \omega^{2}(X, Y)
\end{aligned}
$$


$\log 0$

$$
d \nu \wedge(d \nu \circ J)=-\|\nabla \nu\|^{2} \omega^{1} \wedge \omega^{2}
$$

Além disso, como

$$
\begin{aligned}
X d \nu(J Y)-Y d \nu(J X) & =x_{1} y_{2} e_{1} d \nu\left(J e_{2}\right)+x_{2} y_{1} e_{2} d \nu\left(J e_{1}\right)-x_{1} y_{2} e_{2} d \nu\left(J e_{1}\right)-x_{2} y_{1} e_{1} d \nu\left(J e_{2}\right) \\
& =-x_{1} y_{2} e_{1} d \nu\left(e_{1}\right)+x_{2} y_{1} e_{2} d \nu\left(e_{2}\right)-x_{1} y_{2} e_{2} d \nu\left(e_{2}\right)+x_{2} y_{1} e_{1} d \nu\left(e_{1}\right) \\
& =-\left(e_{1} d \nu\left(e_{1}\right)+e_{2} d \nu\left(e_{2}\right)\right) \omega^{1} \wedge \omega^{2}(X, Y)
\end{aligned}
$$

e

$$
\begin{aligned}
d \nu \circ(J[X, Y]) & =x_{1} y_{2} d \nu\left(J\left[e_{1}, e_{2}\right]\right)+x_{2} y_{1} d \nu\left(J\left[e_{2}, e_{1}\right]\right) \\
& =x_{2} y_{1} d \nu\left(J \nabla_{e_{2}} e_{1}\right)-x_{2} y_{1} d \nu\left(J \nabla_{e_{1}} e_{2}\right)+x_{1} y_{2} d \nu\left(J \nabla_{e_{1}} e_{2}\right)-x_{1} y_{2} d \nu\left(J \nabla_{e_{2}} e_{1}\right) \\
& =x_{2} y_{1} d \nu\left(\nabla_{e_{2}} e_{2}\right)+x_{2} y_{1} d \nu\left(\nabla_{e_{1}} e_{1}\right)-x_{1} y_{2} d \nu\left(\nabla_{e_{1}} e_{1}\right)-x_{1} y_{2} d \nu\left(\nabla_{e_{2}} e_{2}\right) \\
& =-\left(d \nu\left(\nabla_{e_{1}} e_{1}\right)+d \nu\left(\nabla_{e_{2}} e_{2}\right)\right) \omega^{1} \wedge \omega^{2}(X, Y) \\
& =-\left\langle\nabla \nu, \nabla_{e_{1}} e_{1}+\nabla_{e_{2}} e_{2}\right\rangle \omega^{1} \wedge \omega^{2}(X, Y),
\end{aligned}
$$

então

$$
\begin{aligned}
d(d \nu \circ J)(X, Y) & =X d \nu(J Y)-Y d \nu(J X)-d \nu \circ(J[X, Y]) \\
& =-\left(e_{1} d \nu\left(e_{1}\right)+e_{2} d \nu\left(e_{2}\right)-\left\langle\nabla \nu, \nabla_{e_{1}} e_{1}+\nabla_{e_{2}} e_{2}\right\rangle\right) \omega^{1} \wedge \omega^{2}(X, Y) \\
& =-\left(e_{1}\left\langle\nabla \nu, e_{1}\right\rangle-\left\langle\nabla \nu, \nabla_{e_{1}} e_{1}\right\rangle+e_{2}\left\langle\nabla \nu, e_{2}\right\rangle-\left\langle\nabla \nu, \nabla_{e_{2}} e_{2}\right\rangle\right) \omega^{1} \wedge \omega^{2}(X, Y) \\
& =-\left(\left\langle\nabla_{e_{1}} \nabla \nu, e_{1}\right\rangle+\left\langle\nabla_{e_{2}} \nabla \nu, e_{2}\right\rangle\right) \omega^{1} \wedge \omega^{2}(X, Y) \\
& =-\Delta \nu \omega^{1} \wedge \omega^{2}(X, Y),
\end{aligned}
$$

e logo

$$
d(d \nu \circ J)=-\Delta \nu \omega^{1} \wedge \omega^{2} .
$$

Agora note que, como $\left\langle\nabla_{X} e_{1}, \nabla_{Y} e_{2}\right\rangle=\left\langle\nabla_{X} e_{2}, \nabla_{Y} e_{1}\right\rangle=0$, temos

$$
\begin{aligned}
d \omega_{1}^{2}(X, Y) & =X \omega_{1}^{2}(Y)-Y \omega_{1}^{2}(X)-\omega_{1}^{2}([X, Y]) \\
& =X\left\langle\nabla_{Y} e_{1}, e_{2}\right\rangle-Y\left\langle\nabla_{X} e_{1}, e_{2}\right\rangle-\left\langle\nabla_{[X, Y]} e_{1}, e_{2}\right\rangle \\
& =\left\langle\nabla_{X} \nabla_{Y} e_{1}, e_{2}\right\rangle-\left\langle\nabla_{Y} \nabla_{X} e_{1}, e_{2}\right\rangle-\left\langle\nabla_{[X, Y]} e_{1}, e_{2}\right\rangle \\
& =-\left\langle R(X, Y) e_{1}, e_{2}\right\rangle \\
& =-\left\langle R\left(e_{1}, e_{2}\right) e_{1}, e_{2}\right\rangle \omega^{1} \wedge \omega^{2}(X, Y) \\
& =-K \omega^{1} \wedge \omega^{2}(X, Y),
\end{aligned}
$$

e portanto

$$
d \omega_{1}^{2}=-K \omega^{1} \wedge \omega^{2}
$$

Substituindo (3.12), (3.13) e (3.14) em (3.11) temos

$$
d\left(\frac{\nu}{1-\nu^{2}}(d \nu \circ J)+\omega_{1}^{2}\right)=\left(\frac{1+\nu^{2}}{1-\nu^{2}}\left(-\|\nabla \nu\|^{2}\right)+\frac{\nu}{1-\nu^{2}}(-\Delta \nu)-K\right) \omega^{1} \wedge \omega^{2},
$$

e por (3.7) e (3.8) segue que

$$
d\left(\frac{\nu}{1-\nu^{2}}(d \nu \circ J)+\omega_{1}^{2}\right)=0
$$

Daí, como $U$ é simplesmente conexo, a equação (3.10) possui uma solução $\theta: U \rightarrow \mathbb{R}$ que é única a menos de uma constante $\theta_{0}$. Portanto existe $T$ satisfazendo $\|T\|^{2}+\nu^{2}=1$ e (3.9), e pela 
definição de $T$ vemos que é único a menos de uma rotação por um ângulo fixado $\theta_{0}$. Fixamos agora o campo vetorial $T$. Como $T$ não se anula em $U$, podemos definir o operador $A: T U \rightarrow T U$ por

$$
A(X)=-\langle X, \nabla \nu\rangle \frac{T}{\|T\|^{2}}+\langle X, J \nabla \nu\rangle \frac{J T}{\|T\|^{2}} .
$$

O operador $A$ tem as seguintes propriedades:

1. $A(T)=-\nabla \nu$;

2. A tem traço nulo:

$$
\begin{aligned}
\left\langle A\left(\frac{T}{\|T\|}\right), \frac{T}{\|T\|}\right\rangle+\left\langle A\left(\frac{J T}{\|T\|}\right), \frac{J T}{\|T\|}\right\rangle= & -\left\langle\nabla \nu, \frac{T}{\|T\|}\right\rangle\left\langle\frac{T}{\|T\|^{2}}, \frac{T}{\|T\|}\right\rangle \\
& +\left\langle J \nabla \nu, \frac{T}{\|T\|}\right\rangle\left\langle\frac{J T}{\|T\|^{2}}, \frac{T}{\|T\|}\right\rangle \\
& -\left\langle\nabla \nu, \frac{J T}{\|T\|}\right\rangle\left\langle\frac{T}{\|T\|^{2}}, \frac{J T}{\|T\|}\right\rangle \\
& +\left\langle J \nabla \nu, \frac{J T}{\|T\|}\right\rangle\left\langle\frac{J T}{\|T\|^{2}}, \frac{J T}{\|T\|}\right\rangle \\
= & -\frac{1}{\|T\|^{2}}\langle\nabla \nu, T\rangle+\frac{1}{\|T\|^{2}}\langle\nabla \nu, T\rangle \\
= & 0 ;
\end{aligned}
$$

3. A é simétrico:

$$
\begin{aligned}
\left\langle A\left(\frac{T}{\|T\|}\right), \frac{J T}{\|T\|}\right\rangle & =-\left\langle\nabla \nu, \frac{T}{\|T\|}\right\rangle\left\langle\frac{T}{\|T\|^{2}}, \frac{J T}{\|T\|}\right\rangle+\left\langle J \nabla \nu, \frac{T}{\|T\|}\right\rangle\left\langle\frac{J T}{\|T\|^{2}}, \frac{J T}{\|T\|}\right\rangle \\
& =\frac{1}{\|T\|^{2}}\langle J \nabla \nu, T\rangle \\
& =-\left\langle\nabla \nu, \frac{J T}{\|T\|}\right\rangle\left\langle\frac{T}{\|T\|^{2}}, \frac{T}{\|T\|}\right\rangle+\left\langle J \nabla \nu, \frac{J T}{\|T\|}\right\rangle\left\langle\frac{J T}{\|T\|^{2}}, \frac{T}{\|T\|}\right\rangle \\
& =\left\langle A\left(\frac{J T}{\|T\|}\right), \frac{T}{\|T\|}\right\rangle ;
\end{aligned}
$$

4. $A: T U \rightarrow T U$ é o único operador linear com as propriedades 1,2 e 3 acima. Se $\bar{A}: T U \rightarrow T U$ é outro operador linear satisfazendo tais propriedades, então, se $X \in \Gamma(T U)$

$$
\begin{aligned}
\left\langle\bar{A}(X), \frac{T}{\|T\|}\right\rangle & =\left\langle X, \bar{A}\left(\frac{T}{\|T\|}\right)\right\rangle=\left\langle X,-\frac{\nabla \nu}{\|T\|}\right\rangle=\left\langle X, A\left(\frac{T}{\|T\|}\right)\right\rangle \\
& =\left\langle A(X), \frac{T}{\|T\|}\right\rangle
\end{aligned}
$$

e

$$
\begin{aligned}
\left\langle\bar{A}(X), \frac{J T}{\|T\|}\right\rangle & =\left\langle\bar{A}(J X) \frac{T}{\|T\|}\right\rangle=\left\langle J X, \bar{A}\left(\frac{T}{\|T\|}\right)\right\rangle=\left\langle J X,-\frac{\nabla \nu}{\|T\|}\right\rangle \\
& =\left\langle J X, A\left(\frac{T}{\|T\|}\right)\right\rangle=\left\langle A(J X), \frac{T}{\|T\|}\right\rangle=\left\langle A(X), \frac{J T}{\|T\|}\right\rangle
\end{aligned}
$$

$\operatorname{logo} \bar{A}=A$.

Observação 3.1.8. Note que se tomarmos $T_{0}=e^{\theta_{0} J} T$ e definirmos $A_{0}: T U \rightarrow T U$ como em (3.15) a partir de $T_{0}$, então $A_{0}=e^{\theta_{0} J} A$. 
Provaremos agora que $(\langle\rangle, A, T,, \nu)$ satisfaz as equações de compatibilidade para $\mathbb{Q}_{\varepsilon}^{2} \times \mathbb{R}$. Note que pela definição de $T$ temos $\|T\|^{2}+\nu^{2}=1$ e, pela definição de $A$, temos a equação (3.3), e como

$$
\langle A(X), T\rangle=-\langle\nabla \nu, X\rangle\left\langle\frac{T}{\|T\|^{2}}, T\right\rangle=-\langle\nabla \nu, X\rangle=-d \nu(X),
$$

temos a equação (3.4). Além disso, utilizando a equação (3.7),

$$
\begin{aligned}
\operatorname{det} A & =\left[-\frac{1}{\|T\|^{2}}\langle\nabla \nu, T\rangle\right]\left[\frac{1}{\|T\|^{2}}\langle\nabla \nu, T\rangle\right]-\left[\frac{1}{\|T\|^{2}}\langle J \nabla \nu, T\rangle\right]^{2} \\
& =-\frac{1}{\|T\|^{2}}\|\nabla \nu\|^{2} \\
& =\frac{\left(1-\nu^{2}\right)\left(K-\varepsilon \nu^{2}\right)}{\|T\|^{2}} \\
& =K-\varepsilon \nu^{2}
\end{aligned}
$$

e temos a equação (3.1). Falta apenas verificar (3.2). Tome $X, Y \in \Gamma(T U)$. Escrevendo $X=$ $x_{1} T+x_{2} J T, Y=y_{1} T+y_{2} J T$, temos

$$
\nabla_{X} A(Y)-\nabla_{Y} A(X)-A([X, Y])=\left(x_{1} y_{2}-x_{2} y_{1}\right)\left(\nabla_{T} A(J T)-\nabla_{J T} A(T)-A([T, J T]) .\right.
$$

Sabemos que o Hessiano de $\nu$, ou seja, a aplicação $(X, Y) \mapsto\left\langle\nabla_{X} \nabla \nu, Y\right\rangle$, é simétrica (veja [dC79]), logo

$$
\begin{aligned}
\nabla_{T} A(J T)-\nabla_{J T} A(T) & =\nabla_{T} J \nabla \nu+\nabla_{J T} \nabla \nu \\
& =\left\langle J \nabla_{T} \nabla \nu+\nabla_{J T} \nabla \nu, T\right\rangle \frac{T}{\|T\|^{2}}+\left\langle J \nabla_{T} \nabla \nu+\nabla_{J T} \nabla \nu, J T\right\rangle \frac{J T}{\|T\|^{2}} \\
& =\left(\left\langle\nabla_{T} \nabla \nu, T\right\rangle+\left\langle\nabla_{J T} \nabla \nu, J T\right\rangle\right) \frac{J T}{\|T\|^{2}} \\
& =\left(\left\langle\nabla_{\frac{T}{\|T\|}} \nabla \nu, \frac{T}{\|T\|}\right\rangle+\left\langle\nabla_{\frac{J T}{\|T\|}} \nabla \nu, \frac{J T}{\|T\|}\right\rangle\right) J T \\
& =\Delta \nu J T,
\end{aligned}
$$

e usando (3.8) segue que

$$
\nabla_{T} A(J T)-\nabla_{J T} A(T)=\left(2 K \nu-\varepsilon\left(1+\nu^{2}\right) \nu\right) J T .
$$

Agora, como

$$
\begin{aligned}
{[T, J T] } & =\nabla_{T} J T-\nabla_{J T} T \\
& =J \nabla_{T} T-\nu A(J T) \\
& =2 \nu J A(T)
\end{aligned}
$$

temos

$$
\begin{aligned}
A(2 \nu J A(T)) & =\langle A(2 \nu J A(T)), T\rangle \frac{T}{\|T\|^{2}}+\langle A(2 \nu J A(T)), J T\rangle \frac{J T}{\|T\|^{2}} \\
& =\langle 2 \nu J A(T), A(T)\rangle \frac{T}{\|T\|^{2}}+\langle 2 \nu J A(T), A(J T)\rangle \frac{J T}{\|T\|^{2}} \\
& =-2 \nu\langle A(T), A(T)\rangle \frac{J T}{\|T\|^{2}} \\
& =2 \nu\|\nabla \nu\|^{2} \frac{J T}{\|T\|^{2}},
\end{aligned}
$$


e usando (3.7) segue que

$$
A(2 \nu J A(T))=2 \nu\left(K-\varepsilon \nu^{2}\right) J T .
$$

Substituindo (3.17) e (3.18) em (3.16) temos

$$
\begin{aligned}
\nabla_{X} A(Y)-\nabla_{Y} A(X)-A([X, Y]) & =\left(x_{1} y_{2}-x_{2} y_{1}\right)\left(2 K \nu-\varepsilon\left(1+\nu^{2}\right) \nu-2 \nu\left(K-\varepsilon \nu^{2}\right)\right) J T \\
& =\left(x_{1} y_{2}-x_{2} y_{1}\right)(-\varepsilon \nu)\left(1-\nu^{2}\right) J T \\
& =\varepsilon \nu\left(y_{1} x_{2}-x_{1} y_{2}\right)\|T\|^{2} J T .
\end{aligned}
$$

Como

$$
\langle Y, T\rangle X-\langle X, T\rangle Y=\left(y_{1} x_{2}-x_{1} y_{2}\right)\|T\|^{2} J T,
$$

temos (3.2), como queríamos.

Como $\Sigma$ é simplesmente conexa, como na demonstração do teorema 2.2.8, podemos estender $A$ e $T$, definidos em $U$, para toda superfície $\Sigma$ de maneira única, e portanto, pelo Teorema Fundamental 3.1.7, existe uma imersão isométrica mínima $f: \Sigma \rightarrow \mathbb{Q}_{\varepsilon}^{2} \times \mathbb{R}$ com dados compatíveis $(\langle\rangle, A, T,, \nu)$. Seja agora $g: \Sigma \rightarrow \mathbb{Q}_{\varepsilon}^{2} \times \mathbb{R}$ outra imersão isométrica mínima com mesma função ângulo $\nu$. Como $T$ é único a menos de uma rotação por um ângulo $\theta$ e $A$ está definido unicamente em termos de $T$ e $\nu$, pela observação 3.1.8 temos que os dados compatíveis de $\Sigma$ determinados por $g$ são da forma $\left(\langle\rangle,, e^{\theta J} A, e^{\theta J} T, \nu\right)$, para algum ângulo $\theta$, logo $f$ e $g$ são associadas.

\subsection{Superfícies Parametrizadas: Como Fazer as Contas e Proprie- dades}

Seja $x: U \subset \mathbb{R}^{2} \rightarrow M \subset \mathbb{Q}_{\varepsilon}^{2} \times \mathbb{R}$ uma parametrização de uma superfície $M$ em $\mathbb{Q}_{\varepsilon}^{2} \times \mathbb{R} \subset \mathbb{E}^{4}$, com $U$ aberto de $\mathbb{R}^{2}$, e escrevemos $x(u, v)=\left(f_{1}(u, v), f_{2}(u, v), f_{3}(u, v), f_{4}(u, v)\right) \in \mathbb{E}^{4}$, para $(u, v) \in U$. Desta forma temos que $\left\{\frac{\partial}{\partial u}, \frac{\partial}{\partial v}\right\}$ é uma base para $T U$ e $\left\{\frac{\partial x}{\partial u}, \frac{\partial x}{\partial v}\right\}$ é base para $T x(U)$. Sejam $(\langle\rangle, A, T,, \nu)$ os dados compatíveis de $U$ determinados por $x$. Em particular, a métrica em $U$ é dada por

$$
\left\langle\xi_{1}, \xi_{2}\right\rangle=\left\langle d x_{p}\left(\xi_{1}\right), d x_{p}\left(\xi_{2}\right)\right\rangle_{\varepsilon}
$$

onde $p \in U$ e $\xi_{1}, \xi_{2} \in T_{p} U$. Desta forma $x$ é uma imersão isométrica e a matriz da métrica $\langle$,$\rangle é$ dada por

$$
\left.\left.g=\left[\begin{array}{c}
\left\langle\frac{\partial}{\partial u}, \frac{\partial}{\partial u}\right\rangle \\
\left\langle\frac{\partial}{\partial u}, \frac{\partial}{\partial v}\right\rangle
\end{array}\right\rangle \frac{\partial}{\partial u}, \frac{\partial}{\partial v}\right\rangle\right]=\left[\begin{array}{ll}
\left\langle\frac{\partial x}{\partial v}, \frac{\partial x}{\partial v}, \frac{\partial x}{\partial u}\right\rangle_{\varepsilon} & \left\langle\frac{\partial x}{\partial u}, \frac{\partial x}{\partial v}\right\rangle_{\varepsilon} \\
\left\langle\frac{\partial x}{\partial u}, \frac{\partial x}{\partial v}\right\rangle_{\varepsilon} & \left\langle\frac{\partial x}{\partial v}, \frac{\partial x}{\partial v}\right\rangle_{\varepsilon}
\end{array}\right] .
$$

Assim, se $\xi=\left(\xi^{1}, \xi^{2}\right) \in \mathbb{R}^{2}$, então $\langle d x(\xi), d x(\xi)\rangle_{\varepsilon}=\langle\xi, \xi\rangle=\xi^{t} g \xi$. Como já vimos anteriormente, o vetor $\bar{N}$ normal a $M$ e a $\mathbb{Q}_{\varepsilon}^{2} \times \mathbb{R}$ em $\mathbb{E}^{4}$ é dado pelo vetor posição de $x(U)$ no primeiro fator, ou seja,

$$
\bar{N}(u, v)=\left(f_{1}(u, v), f_{2}(u, v), f_{3}(u, v), 0\right) .
$$

Vamos agora calcular o vetor $N$ normal a $x(u)$ em $\mathbb{Q}_{\varepsilon}^{2} \times \mathbb{R}$. Para isso, usaremos a seguinte generalização do produto vetorial de $\mathbb{R}^{3}$ para $\mathbb{E}^{4}$.

Definição 3.2.1. Sejam $\xi_{1}, \xi_{2}, \xi_{3} \in \mathbb{E}^{4}$. Definimos $\xi_{1} \wedge \xi_{2} \wedge \xi_{3}$ como a única solução de

$$
\left\langle\xi_{1} \wedge \xi_{2} \wedge \xi_{3}, \eta\right\rangle_{\varepsilon}=\operatorname{det}\left[\begin{array}{c}
\xi_{1} \\
\xi_{2} \\
\xi_{3} \\
\eta
\end{array}\right]
$$


Claramente a aplicação acima é trilinear e anti-simétrica. Se $\xi_{i}$ é um vetor de $\mathbb{E}^{4}$ e $\left(\xi_{i}^{0}, \xi_{i}^{1}, \xi_{i}^{2}, \xi_{i}^{3}\right)$ são suas coordenadas na base canônica de $\mathbb{E}^{4},\left\{E_{0}, E_{1}, E_{2}, E_{3}\right\}$, então o vetor $\xi_{1} \wedge \xi_{2} \wedge \xi_{3}$ pode ser calculado explicitamente através da fórmula

$$
\xi_{1} \wedge \xi_{2} \wedge \xi_{3}=-\operatorname{det}\left[\begin{array}{cccc}
\varepsilon E_{0} & E_{1} & E_{2} & E_{3} \\
\xi_{1}^{0} & \xi_{1}^{1} & \xi_{1}^{2} & \xi_{1}^{3} \\
\xi_{2}^{0} & \xi_{2}^{1} & \xi_{2}^{2} & \xi_{2}^{3} \\
\xi_{3}^{0} & \xi_{3}^{1} & \xi_{3}^{2} & \xi_{3}^{3}
\end{array}\right]
$$

De fato, se $\eta$ é vetor de $\mathbb{E}^{4}$, como

$$
\begin{aligned}
\operatorname{det}\left[\begin{array}{cccc}
\varepsilon E_{0} & E_{1} & E_{2} & E_{3} \\
\xi_{1}^{0} & \xi_{1}^{1} & \xi_{1}^{2} & \xi_{1}^{3} \\
\xi_{2}^{0} & \xi_{2}^{1} & \xi_{2}^{2} & \xi_{2}^{3} \\
\xi_{3}^{0} & \xi_{3}^{1} & \xi_{3}^{2} & \xi_{3}^{3}
\end{array}\right]= & \varepsilon \operatorname{det}\left[\begin{array}{lll}
\xi_{1}^{1} & \xi_{1}^{2} & \xi_{1}^{3} \\
\xi_{2}^{1} & \xi_{2}^{2} & \xi_{2}^{3} \\
\xi_{3}^{1} & \xi_{3}^{2} & \xi_{3}^{3}
\end{array}\right] E_{0}-\operatorname{det}\left[\begin{array}{lll}
\xi_{1}^{0} & \xi_{1}^{2} & \xi_{1}^{3} \\
\xi_{2}^{0} & \xi_{2}^{2} & \xi_{2}^{3} \\
\xi_{3}^{0} & \xi_{3}^{2} & \xi_{3}^{3}
\end{array}\right] E_{1} \\
& +\operatorname{det}\left[\begin{array}{lll}
\xi_{1}^{0} & \xi_{1}^{1} & \xi_{1}^{3} \\
\xi_{2}^{0} & \xi_{2}^{1} & \xi_{2}^{3} \\
\xi_{3}^{0} & \xi_{3}^{1} & \xi_{3}^{3}
\end{array}\right] E_{2}-\operatorname{det}\left[\begin{array}{lll}
\xi_{1}^{0} & \xi_{1}^{1} & \xi_{1}^{2} \\
\xi_{2}^{0} & \xi_{2}^{1} & \xi_{2}^{2} \\
\xi_{3}^{0} & \xi_{3}^{1} & \xi_{3}^{2}
\end{array}\right] E_{3},
\end{aligned}
$$

utilizando (3.19) temos

$$
\begin{aligned}
\left\langle\xi_{1} \wedge \xi_{2} \wedge \xi_{3}, \eta\right\rangle_{\varepsilon}= & -\operatorname{det}\left[\begin{array}{lll}
\xi_{1}^{1} & \xi_{1}^{2} & \xi_{1}^{3} \\
\xi_{2}^{1} & \xi_{2}^{2} & \xi_{2}^{3} \\
\xi_{3}^{1} & \xi_{3}^{2} & \xi_{3}^{3}
\end{array}\right] \eta^{0}+\operatorname{det}\left[\begin{array}{ccc}
\xi_{1}^{0} & \xi_{1}^{2} & \xi_{1}^{3} \\
\xi_{2}^{0} & \xi_{2}^{2} & \xi_{2}^{3} \\
\xi_{3}^{0} & \xi_{3}^{2} & \xi_{3}^{3}
\end{array}\right] \eta^{1} \\
& -\operatorname{det}\left[\begin{array}{ccc}
\xi_{1}^{0} & \xi_{1}^{1} & \xi_{1}^{3} \\
\xi_{2}^{0} & \xi_{2}^{1} & \xi_{2}^{3} \\
\xi_{3}^{0} & \xi_{3}^{1} & \xi_{3}^{3}
\end{array}\right] \eta^{2}+\operatorname{det}\left[\begin{array}{lll}
\xi_{1}^{0} & \xi_{1}^{1} & \xi_{1}^{2} \\
\xi_{2}^{0} & \xi_{2}^{1} & \xi_{2}^{2} \\
\xi_{3}^{0} & \xi_{3}^{1} & \xi_{3}^{2}
\end{array}\right] \eta^{3} \\
= & \operatorname{det}\left[\begin{array}{c}
\xi_{1} \\
\xi_{2} \\
\xi_{3} \\
\eta
\end{array}\right]
\end{aligned}
$$

como queríamos. Note que $\xi_{1} \wedge \xi_{2} \wedge \xi_{3} \in\left[\xi_{1}, \xi_{2}, \xi_{3}\right]^{\perp}$ e $\xi_{1} \wedge \xi_{2} \wedge \xi_{3}=0$ se e somente se $\left\{\xi_{1}, \xi_{2}, \xi_{3}\right\}$ é linearmente dependente.

Pela definição temos que

$$
0<\left\langle\bar{N} \wedge \frac{\partial x}{\partial u} \wedge \frac{\partial x}{\partial v}, \bar{N} \wedge \frac{\partial x}{\partial u} \wedge \frac{\partial x}{\partial v}\right\rangle_{\varepsilon}=\operatorname{det}\left[\begin{array}{c}
\bar{N} \\
\frac{\partial x}{\partial u} \\
\frac{\partial x}{\partial v} \\
\bar{N} \wedge \frac{\partial x}{\partial u} \wedge \frac{\partial x}{\partial v}
\end{array}\right]
$$

Portanto podemos utilizar (3.19) para definir o vetor $N$ :

$$
N=\frac{\bar{N} \wedge \frac{\partial x}{\partial u} \wedge \frac{\partial x}{\partial v}}{\left\|\bar{N} \wedge \frac{\partial x}{\partial u} \wedge \frac{\partial x}{\partial v}\right\|},
$$

e pelo que vimos segue que $\left\{\bar{N}, \frac{\partial x}{\partial u}, \frac{\partial x}{\partial v}, N\right\}$ forma uma base ortonormal positiva.

Vamos agora calcular a matriz do operador $A$ na base $\left\{\frac{\partial}{\partial u}, \frac{\partial}{\partial v}\right\}$ em $T U$. Pelo que vimos na 
demonstração do Teorema Fundamental 2.2.8, temos que

$$
\left\langle A\left(\frac{\partial}{\partial u}\right), \frac{\partial}{\partial u}\right\rangle=\left\langle\frac{\partial x}{\partial u}\left(\frac{\partial x}{\partial u}\right), N\right\rangle_{\varepsilon}=\left\langle\frac{\partial^{2} x}{\partial u^{2}}, N\right\rangle_{\varepsilon} .
$$

Analogamente temos

$$
\left\langle A\left(\frac{\partial}{\partial v}\right), \frac{\partial}{\partial v}\right\rangle=\left\langle\frac{\partial^{2} x}{\partial v^{2}}, N\right\rangle_{\varepsilon}
$$

e

$$
\left\langle A\left(\frac{\partial}{\partial u}\right), \frac{\partial}{\partial v}\right\rangle=\left\langle\frac{\partial^{2} x}{\partial u \partial v}, N\right\rangle_{\varepsilon},
$$

logo segue que, na base $\left\{\frac{\partial}{\partial u}, \frac{\partial}{\partial v}\right\}$, denotando por $b$ a matriz

$$
b=\left[\begin{array}{cc}
\left\langle\frac{\partial^{2} x}{\partial u^{2}}, N\right\rangle_{\varepsilon} & \left\langle\frac{\partial^{2} x}{\partial u \partial v}, N\right\rangle_{\varepsilon} \\
\left\langle\frac{\partial^{2} x}{\partial u \partial v}, N\right\rangle_{\varepsilon} & \left\langle\frac{\partial^{2} x}{\partial v^{2}}, N\right\rangle_{\varepsilon}
\end{array}\right]
$$

a matriz do operador $A$ é dado por

$$
A=g^{-1} b
$$

ou seja,

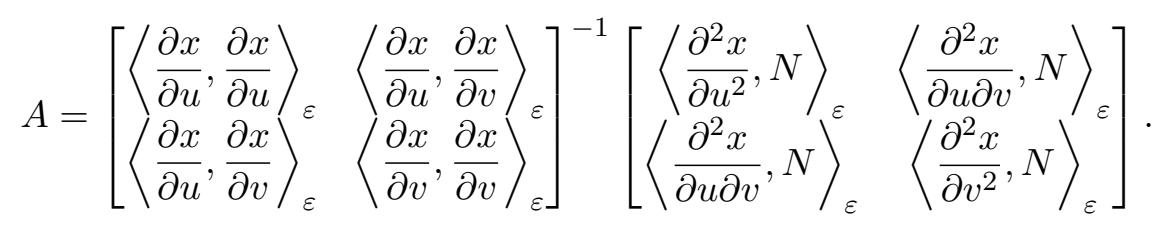

Suponha agora que $x$ seja parametrização conforme, ou seja,

$$
\left\langle\frac{\partial x}{\partial u}, \frac{\partial x}{\partial u}\right\rangle_{\varepsilon}-\left\langle\frac{\partial x}{\partial v}, \frac{\partial x}{\partial v}\right\rangle_{\varepsilon}=\left\langle\frac{\partial x}{\partial u}, \frac{\partial x}{\partial v}\right\rangle_{\varepsilon}=0 .
$$

Então existe uma função $\sigma: U \rightarrow \mathbb{R}$ tal que

$$
e^{2 \sigma}=\left\langle\frac{\partial x}{\partial u}, \frac{\partial x}{\partial u}\right\rangle_{\varepsilon}
$$

daí $g=e^{2 \sigma} I$ e $A=e^{-2 \sigma} b$. Portanto temos que

$$
\begin{aligned}
& 2 H=\operatorname{tr}(A)=\operatorname{tr}\left(e^{-2 \sigma} b\right)=e^{-2 \sigma}\left(\left\langle\frac{\partial^{2} x}{\partial u^{2}}, N\right\rangle_{\varepsilon}+\left\langle\frac{\partial^{2} x}{\partial v^{2}}, N\right\rangle_{\varepsilon}\right) . \\
\Rightarrow & \langle\Delta x, N\rangle_{\varepsilon}=2 e^{2 \sigma} H .
\end{aligned}
$$


Além disso, temos que

$$
\begin{aligned}
& \left\langle\frac{\partial x}{\partial u}, \frac{\partial x}{\partial u}\right\rangle_{\varepsilon}=\left\langle\frac{\partial x}{\partial v}, \frac{\partial x}{\partial v}\right\rangle_{\varepsilon} \\
\Rightarrow & \frac{\partial}{\partial u}\left\langle\frac{\partial x}{\partial u}, \frac{\partial x}{\partial u}\right\rangle_{\varepsilon}=\frac{\partial}{\partial u}\left\langle\frac{\partial x}{\partial v}, \frac{\partial x}{\partial v}\right\rangle_{\varepsilon} \\
\Rightarrow & \left\langle\frac{\partial^{2} x}{\partial u^{2}}, \frac{\partial x}{\partial u}\right\rangle_{\varepsilon}=\left\langle\frac{\partial^{2} x}{\partial v^{2}}, \frac{\partial x}{\partial v}\right\rangle_{\varepsilon} \\
\Rightarrow & \left\langle\frac{\partial^{2} x}{\partial u^{2}}, \frac{\partial x}{\partial u}\right\rangle_{\varepsilon}=\frac{\partial}{\partial v}\left\langle\frac{\partial x}{\partial u}, \frac{\partial x}{\partial v}\right\rangle_{\varepsilon}-\left\langle\frac{\partial x}{\partial u}, \frac{\partial^{2} x}{\partial u^{2}}\right\rangle_{\varepsilon},
\end{aligned}
$$

e portanto,

$$
\left\langle\Delta x, \frac{\partial x}{\partial u}\right\rangle_{\varepsilon}=0
$$

Analogamente,

$$
\left\langle\Delta x, \frac{\partial x}{\partial v}\right\rangle_{\varepsilon}=0
$$

Portanto, se $x$ é imersão mínima, $\langle\Delta x, N\rangle_{\varepsilon}=0$, logo $\Delta x$ é proporcional ao vetor $\bar{N}$.

\subsection{Superfícies Conjugadas em $\mathbb{S}^{2} \times \mathbb{R}$}

Nesta seção vamos mostrar que as superfícies conjugadas de onduloides são helicoides.

Helicoides: São obtidos através da rotação, com velocidade constante, ao longo de uma translação no eixo vertical, também com velocidade constante, de uma geodésica de $\mathbb{S}^{2}$. Para $\beta \neq 0$, seja o helicoide $\mathcal{H}_{\beta}$ dado pela seguinte imersão:

$$
x(u, v)=\left[\begin{array}{c}
\operatorname{sen} \phi(u) \cos \beta v \\
\operatorname{sen} \phi(u) \operatorname{sen} \beta v \\
\cos \phi(u) \\
v
\end{array}\right],
$$

onde a função $\phi$ satisfaz

$$
\phi^{\prime}(u)^{2}=1+\beta^{2} \operatorname{sen}^{2} \phi(u), \quad \phi^{\prime \prime}(u)=\beta^{2} \operatorname{sen} \phi(u) \cos \phi(u) .
$$

Note que

$$
\frac{\partial x}{\partial u}=\left(\phi^{\prime}(u) \cos \phi(u) \cos \beta v, \phi^{\prime}(u) \cos \phi(u) \operatorname{sen} \beta v,-\phi^{\prime}(u) \operatorname{sen} \phi(u), 0\right)
$$

e

$$
\frac{\partial x}{\partial v}=(-\operatorname{sen} \phi(u) \beta \operatorname{sen} \beta v, \operatorname{sen} \phi(u) \beta \cos \beta v, 0,1) .
$$

Temos também que

$$
\left\langle\frac{\partial x}{\partial u}, \frac{\partial x}{\partial u}\right\rangle_{1}=\phi^{\prime}(u)^{2}, \quad\left\langle\frac{\partial x}{\partial u}, \frac{\partial x}{\partial v}\right\rangle_{1}=0, \quad\left\langle\frac{\partial x}{\partial v}, \frac{\partial x}{\partial v}\right\rangle_{1}=1+\beta^{2} \operatorname{sen}^{2} \phi(u),
$$

logo a imersão $x$ é conforme. Podemos assumir que $\phi(0)=0$ e $\phi^{\prime}(u)>0$.

Pelo que vimos na seção 3.2 , o normal $\bar{N}$ a $\mathbb{S}^{2} \times \mathbb{R}$ em $\mathbb{R}^{4}$ é o vetor posição de $\mathbb{S}^{2}$ em $\mathbb{R}^{4}$, ou seja, 


$$
\bar{N}(u, v)=\left[\begin{array}{c}
\operatorname{sen} \phi(u) \cos \beta v \\
\operatorname{sen} \phi(u) \operatorname{sen} \beta v \\
\cos \phi(u) \\
0
\end{array}\right]
$$

Além disso, o normal $N$ a $\mathcal{H}_{\beta}$ em $\mathbb{S}^{2} \times \mathbb{R}$ é

$$
N(u, v)=\frac{1}{\phi^{\prime}(u)}\left[\begin{array}{c}
\operatorname{sen} \beta v \\
-\cos \beta v \\
0 \\
\beta \operatorname{sen} \phi(u)
\end{array}\right] .
$$

Vamos calcular o operador forma $A_{\mathcal{H}_{\beta}}$ de $\mathcal{H}_{\beta}$. Temos:

$$
\begin{gathered}
\frac{\partial^{2} x}{\partial u^{2}}=\left[\begin{array}{c}
\phi^{\prime \prime}(u) \cos \phi(u) \cos \beta v-\phi^{\prime}(u)^{2} \operatorname{sen} \phi(u) \cos \beta v \\
\phi^{\prime \prime}(u) \cos \phi(u) \operatorname{sen} \beta v-\phi^{\prime}(u)^{2} \operatorname{sen} \phi(u) \operatorname{sen} \beta v \\
-\phi^{\prime \prime}(u) \operatorname{sen} \phi(u)-\phi^{\prime}(u)^{2} \cos \phi(u) \\
0
\end{array}\right], \\
\frac{\partial^{2} x}{\partial v^{2}}=\left[\begin{array}{c}
-\operatorname{sen} \phi(u) \beta^{2} \cos \beta v \\
-\operatorname{sen} \phi(u) \beta^{2} \operatorname{sen} \beta v \\
0 \\
0
\end{array}\right] \\
\frac{\partial^{2} x}{\partial u \partial v}=\left[\begin{array}{c}
-\phi^{\prime}(u) \cos \phi(u) \beta \operatorname{sen} \beta v \\
\phi^{\prime}(u) \cos \phi(u) \beta \cos \beta v \\
0 \\
0
\end{array}\right]
\end{gathered}
$$

Portanto,

$$
\left\langle\frac{\partial^{2} x}{\partial u^{2}}, N\right\rangle_{1}=0, \quad\left\langle\frac{\partial^{2} x}{\partial v^{2}}, N\right\rangle_{1}=0,\left\langle\frac{\partial^{2} x}{\partial u \partial v}, N\right\rangle_{1}=-\beta \cos \phi(u) .
$$

Daí, $A_{\mathcal{H}_{\beta}}$ é dado na base $\left\{\frac{\partial}{\partial u}, \frac{\partial}{\partial v}\right\}$ por

$$
\begin{aligned}
A_{\mathcal{H}_{\beta}} & =\left[\begin{array}{cc}
\frac{1}{\phi^{\prime}(u)^{2}} & 0 \\
0 & \frac{1}{\phi^{\prime}(u)^{2}}
\end{array}\right]\left[\begin{array}{cc}
0 & -\beta \cos \phi(u) \\
-\beta \cos \phi(u) & 0
\end{array}\right] \\
& =-\frac{\beta \cos \phi(u)}{\phi^{\prime}(u)^{2}}\left[\begin{array}{ll}
0 & 1 \\
1 & 0
\end{array}\right] .
\end{aligned}
$$

Além disso,

$$
\nu_{\mathcal{H}_{\beta}}=\left\langle\frac{\partial}{\partial t}, N\right\rangle_{1}=\frac{\beta \operatorname{sen} \phi(u)}{\phi^{\prime}(u)}
$$

e

$$
\begin{aligned}
d x\left(T_{\mathcal{H}_{\beta}}\right) & =\frac{\partial}{\partial t}-\nu N \\
& =(0,0,0,1)-\frac{\beta \operatorname{sen} \phi(u)}{\phi^{\prime}(u)^{2}}(\operatorname{sen} \beta v,-\cos \beta v, 0, \beta \operatorname{sen} \phi(u)) \\
& =\frac{1}{\phi^{\prime}(u)^{2}} \frac{\partial x}{\partial v}
\end{aligned}
$$


$\log 0$

$$
T_{\mathcal{H}_{\beta}}=\frac{1}{\phi^{\prime}(u)^{2}} \frac{\partial}{\partial v} .
$$

Note que quando $\beta=0$, a fórmula define um cilindro vertical $\mathbb{S}^{1} \times \mathbb{R}$, e quando $\beta \rightarrow \infty$, a superfície converge para uma folheação por esferas horizontais $\mathbb{S}^{2} \times\{t\}$.

Onduloides: São superfícies invariantes por uma rotação de $\mathbb{S}^{2}$ e folheadas por círculos em $\mathbb{S}^{2}$. Para $\alpha>1$ ou $\alpha<-1$, seja o onduloide $\mathcal{U}_{\alpha}$ dado pela seguinte imersão:

$$
x(u, v)=\left[\begin{array}{c}
\operatorname{sen} \psi(u) \cos \alpha v \\
\operatorname{sen} \psi(u) \operatorname{sen} \alpha v \\
\cos \psi(u) \\
u
\end{array}\right],
$$

onde a função $\psi$ satisfaz

$$
1+\psi^{\prime}(u)^{2}=\alpha^{2} \operatorname{sen}^{2} \psi(u), \quad \psi^{\prime \prime}(u)=\alpha^{2} \operatorname{sen} \psi(u) \cos \psi(u) .
$$

Note que

$$
\frac{\partial x}{\partial u}=\left(\psi^{\prime}(u) \cos \psi(u) \cos \alpha v, \psi^{\prime}(u) \cos \psi(u) \operatorname{sen} \alpha v,-\psi^{\prime}(u) \operatorname{sen} \psi(u), 1\right)
$$

$$
\frac{\partial x}{\partial v}=(-\operatorname{sen} \psi(u) \alpha \operatorname{sen} \alpha v, \operatorname{sen} \psi(u) \alpha \cos \alpha v, 0,0) .
$$

Temos também que

$$
\left\langle\frac{\partial x}{\partial u}, \frac{\partial x}{\partial u}\right\rangle_{1}=\psi^{\prime}(u)^{2}+1, \quad\left\langle\frac{\partial x}{\partial u}, \frac{\partial x}{\partial v}\right\rangle_{1}=0, \quad\left\langle\frac{\partial x}{\partial v}, \frac{\partial x}{\partial v}\right\rangle_{1}=\alpha^{2} \operatorname{sen}^{2} \psi(u),
$$

logo a imersão $x$ é conforme. Vamos assumir que $\psi^{\prime}(0)=0$, e $\phi(u) \in(0, \pi)$ e $\cos \psi(u)>0$.

O normal $\bar{N}$ a $\mathbb{S}^{2} \times \mathbb{R}$ em $\mathbb{R}^{4}$ é o vetor posição de $\mathbb{S}^{2}$ em $\mathbb{R}^{4}$, ou seja,

$$
\bar{N}(u, v)=\left[\begin{array}{c}
\operatorname{sen} \psi(u) \cos \alpha v \\
\operatorname{sen} \psi(u) \operatorname{sen} \alpha v \\
\cos \psi(u) \\
0
\end{array}\right] .
$$

Além disso, pelo que vimos na seção 3.2 , o normal $N$ a $\mathcal{U}_{\alpha}$ em $\mathbb{S}^{2} \times \mathbb{R}$ é

$$
N(u, v)=\frac{1}{\alpha \operatorname{sen} \psi(u)}\left[\begin{array}{c}
-\cos \psi(u) \cos \alpha v \\
-\cos \psi(u) \operatorname{sen} \alpha v \\
\operatorname{sen} \psi(u) \\
\psi^{\prime}(u)
\end{array}\right] .
$$

Vamos calcular o operador forma $A_{\mathcal{U}_{\alpha}}$ de $\mathcal{U}_{\alpha}$. Temos:

$$
\begin{gathered}
\frac{\partial^{2} x}{\partial u^{2}}=\left[\begin{array}{c}
\psi^{\prime \prime}(u) \cos \psi(u) \cos \alpha v-\psi^{\prime}(u)^{2} \operatorname{sen} \psi(u) \cos \alpha v \\
\psi^{\prime \prime}(u) \cos \psi(u) \operatorname{sen} \alpha v-\psi^{\prime}(u)^{2} \operatorname{sen} \psi(u) \operatorname{sen} \alpha v \\
-\psi^{\prime \prime}(u) \operatorname{sen} \psi(u)-\psi^{\prime}(u)^{2} \cos \psi(u) \\
0
\end{array}\right], \\
\frac{\partial^{2} x}{\partial v^{2}}=\left[\begin{array}{c}
-\operatorname{sen} \psi(u) \alpha^{2} \cos \alpha v \\
-\operatorname{sen} \psi(u) \alpha^{2} \operatorname{sen} \alpha v \\
0 \\
0
\end{array}\right]
\end{gathered}
$$




$$
\frac{\partial^{2} x}{\partial u \partial v}=\left[\begin{array}{c}
-\psi^{\prime}(u) \cos \psi(u) \alpha \operatorname{sen} \alpha v \\
\psi^{\prime}(u) \cos \psi(u) \alpha \cos \alpha v \\
0 \\
0
\end{array}\right]
$$

Portanto,

$$
\left\langle\frac{\partial^{2} x}{\partial u^{2}}, N\right\rangle_{1}=-\alpha \cos \psi(u), \quad\left\langle\frac{\partial^{2} x}{\partial v^{2}}, N\right\rangle_{1}=\alpha \cos \psi(u),\left\langle\frac{\partial^{2} x}{\partial u \partial v}, N\right\rangle_{1}=0 .
$$

Daí, $A_{\mathcal{U}_{\alpha}}$ é dado na base $\left\{\frac{\partial}{\partial u}, \frac{\partial}{\partial v}\right\}$ por

$$
A_{\mathcal{U}_{\alpha}}=\left[\begin{array}{cc}
\frac{1}{1+\psi^{\prime}(u)^{2}} & 0 \\
0 & \frac{1}{1+\psi^{\prime}(u)^{2}}
\end{array}\right]\left[\begin{array}{cc}
-\alpha \cos \psi(u) & 0 \\
0 & \alpha \cos \psi(u)
\end{array}\right]=-\frac{\alpha \cos \psi(u)}{1+\psi^{\prime}(u)^{2}}\left[\begin{array}{cc}
1 & 0 \\
0 & -1
\end{array}\right] .
$$

Além disso,

$$
\nu_{\mathcal{U}_{\alpha}}=\left\langle\frac{\partial}{\partial t}, N\right\rangle_{1}=\frac{\psi^{\prime}(u)}{\alpha \operatorname{sen} \psi(u)}
$$

$\mathrm{e}$

$$
\begin{aligned}
d x\left(T_{\mathcal{U}_{\alpha}}\right) & =\frac{\partial}{\partial t}-\nu N \\
& =(0,0,0,1)-\frac{\psi^{\prime}(u)}{\alpha \operatorname{sen} \psi(u)}\left(-\cos \psi(u) \cos \alpha v,-\cos \psi(u) \operatorname{sen} \alpha v, \operatorname{sen} \psi(u), \psi^{\prime}(u)\right) \\
& =\frac{1}{1+\psi^{\prime}(u)^{2}} \frac{\partial x}{\partial u}
\end{aligned}
$$

$\log \mathrm{O}$

$$
T_{\mathcal{U}_{\alpha}}=\frac{1}{1+\psi^{\prime}(u)^{2}} \frac{\partial}{\partial u} .
$$

Note que quando $\alpha= \pm 1$, a fórmula define um cilindro vertical $\mathbb{S}^{1} \times \mathbb{R}$, e quando $\alpha \rightarrow \infty$, a superfície converge para uma folheação por esferas horizontais $\mathbb{S}^{2} \times\{t\}$.

Proposição 3.3.1. A superfície conjugada do onduloide $\mathcal{U}_{\alpha}$ é o helicoide $\mathcal{H}_{\beta}$ tal que $\alpha^{2}=1+\beta^{2}$ e $\alpha, \beta$ com mesmo sinal.

Demonstração. Sejam $y_{1}(u)=\alpha \cos \psi(u)$ e $y_{2}(u)=\beta \cos \phi(u)$. Note que

$$
\begin{aligned}
\left(y_{1}(u)^{2}-\alpha^{2}\right)\left(y_{1}(u)^{2}-\beta^{2}\right) & =\left(\alpha^{2} \cos ^{2} \psi(u)-\alpha^{2}\right)\left(\alpha^{2} \cos ^{2} \psi(u)-\alpha^{2}+1\right) \\
& =\left(-\alpha^{2} \operatorname{sen}^{2} \psi(u)\right)\left(-\alpha^{2} \operatorname{sen}^{2} \psi(u)+1\right) \\
& =\left(-\alpha^{2} \operatorname{sen}^{2} \psi(u)\right)\left(-\psi^{\prime}(u)^{2}\right) \\
& =y_{1}^{\prime}(u)^{2} .
\end{aligned}
$$

Da mesma forma temos

$$
\left(y_{2}(u)^{2}-\alpha^{2}\right)\left(y_{2}(u)^{2}-\beta^{2}\right)=y_{2}^{\prime}(u)^{2} .
$$

Portanto $y_{1}$ e $y_{2}$ são soluções da equação

$$
\left(y^{\prime}\right)^{2}=\left(y^{2}-\alpha^{2}\right)\left(y^{2}-\beta^{2}\right) .
$$

Derivando (3.20), obtemos

$$
y^{\prime \prime}=y\left(2 y^{2}-\alpha^{2}-\beta^{2}\right)
$$


logo $y_{1}$ e $y_{2}$ também são soluções de (3.21). Como

$$
1+\psi^{\prime}(u)^{2}=\alpha^{2} \operatorname{sen}^{2} \psi(u)=\alpha^{2}\left(1-\cos ^{2} \psi(u)\right)=\alpha^{2}-y_{1}(u)^{2},
$$

usando o fato de que $\psi^{\prime}(0)=0$ e $\alpha^{2}-1=\beta^{2}$, temos

$$
y_{1}(0)^{2}=\beta^{2} .
$$

Substituindo em (3.20) temos

$$
y_{1}^{\prime}(0)=0
$$

Além disso, como $\phi(0)=0$, temos

$$
y_{2}(0)=\beta \cos \phi(0)=\beta \text {. }
$$

Substituindo em (3.20) temos

$$
y_{2}^{\prime}(0)=0 .
$$

Sabemos que $\cos \psi(0)>0, \log y_{1}(0)$ tem o mesmo sinal de $\alpha$, e como $\alpha$ e $\beta$ têm o mesmo sinal, por $(3.22)$ e $(3.23)$ segue que $y_{1}(0)=y_{2}(0)$. Pelo teorema de existência e unicidade de soluções de EDO concluímos que $y_{1}=y_{2}$.

Precisamos mostrar que $\mathcal{U}_{\alpha}$ e $\mathcal{H}_{\beta}$ são localmente isométricos, $A_{\mathcal{H}_{\beta}}=J A_{\mathcal{U}_{\alpha}}, T_{\mathcal{H}_{\beta}}=J T_{\mathcal{U}_{\alpha}}$ e $\nu_{\mathcal{H}_{\beta}}=\nu_{\mathcal{U}_{\alpha}}$.

1. Como

$$
\begin{aligned}
\phi^{\prime}(u) & =1+\beta^{2} \operatorname{sen}^{2} \phi(u)=1+\beta^{2}-\beta^{2} \cos ^{2} \phi(u)=\alpha^{2}-y_{2}(u)^{2}=\alpha^{2}-y_{1}(u)^{2} \\
& =\alpha^{2}-\alpha^{2} \cos ^{2} \psi(u)=\alpha^{2} \operatorname{sen}^{2} \psi(u)=1+\psi^{\prime}(u)^{2},
\end{aligned}
$$

segue que $\mathcal{U}_{\alpha}$ e $\mathcal{H}_{\beta}$ são localmente isométricos;

2. Utilizando o item anterior, $\operatorname{como} \beta \cos \phi(u)=y_{1}(u)=y_{2}(u)=\alpha \cos \psi(u)$,

$$
\begin{aligned}
A_{\mathcal{H}_{\beta}} & =-\frac{\beta \cos \phi(u)}{\phi^{\prime}(u)^{2}}\left[\begin{array}{ll}
0 & 1 \\
1 & 0
\end{array}\right]=-\frac{\alpha \cos \psi(u)}{1+\psi^{\prime}(U)^{2}}\left[\begin{array}{ll}
0 & 1 \\
1 & 0
\end{array}\right]=-\frac{\alpha \cos \psi(u)}{1+\psi^{\prime}(U)^{2}}\left[\begin{array}{cc}
0 & -1 \\
1 & 0
\end{array}\right]\left[\begin{array}{cc}
1 & 0 \\
0 & -1
\end{array}\right] \\
& =J A_{\mathcal{U}_{\alpha}}
\end{aligned}
$$

3.

$$
T_{\mathcal{H}_{\beta}}=\frac{1}{\phi^{\prime}(u)^{2}} \frac{\partial}{\partial v}=\frac{1}{1+\psi^{\prime}(u)^{2}} J \frac{\partial}{\partial u}=J T_{\mathcal{U}_{\alpha}}
$$

4. Temos

$$
\nu_{\mathcal{U}_{\alpha}}=\frac{\psi^{\prime}(u)}{\alpha \operatorname{sen} \psi(u)}=-\frac{y_{1}^{\prime}(u)}{\alpha^{2} \operatorname{sen}^{2} \psi(u)}=-\frac{y_{1}^{\prime}(u)}{\alpha^{2}-\alpha^{2} \cos ^{2} \psi(u)}=-\frac{y_{1}^{\prime}(u)}{\alpha^{2}-y_{1}(u)^{2}}
$$

e

$$
\begin{aligned}
\nu_{\mathcal{H}_{\beta}} & =\frac{\beta \operatorname{sen} \phi(u)}{\phi^{\prime}(u)}=\frac{\beta \phi^{\prime}(u) \operatorname{sen} \phi(u)}{\phi^{\prime}(u)^{2}}=-\frac{y_{2}^{\prime}(u)}{1+\beta^{2} \operatorname{sen}^{2} \phi(u)}=-\frac{y_{2}^{\prime}(u)}{1+\beta^{2}-\beta^{2} \cos ^{2} \phi(u)} \\
& =-\frac{y_{2}^{\prime}(u)}{\alpha^{2}-y_{2}(u)^{2}}
\end{aligned}
$$

$\log 0 \nu_{\mathcal{H}_{\beta}}=\nu_{\mathcal{U}_{\alpha}}$

Portanto segue que $\mathcal{H}_{\beta}$ é superfície conjugada de $\mathcal{U}_{\alpha}$, como queríamos. 


\subsection{Superfícies Conjugadas em $\mathbb{H}^{2} \times \mathbb{R}$}

Nesta seção mostraremos que helicoides são superfícies conjugadas de catenoides ou superfícies folheadas por curvas horizontais de curvatura constante.

Helicoides: Como na seção anterior, helicoides são obtidos através da rotação, com velocidade constante, ao longo de uma translação no eixo vertical, também com velocidade constante, de uma geodésica de $\mathbb{H}^{2}$. Para $\beta \neq 0$, seja o helicoide $\mathcal{H}_{\beta}$ dado pela seguinte imersão:

$$
x(u, v)=\left[\begin{array}{c}
\cosh \phi(u) \\
\operatorname{senh} \phi(u) \cos \beta v \\
\operatorname{senh} \phi(u) \operatorname{sen} \beta v \\
v
\end{array}\right],
$$

onde a função $\phi$ satisfaz

$$
\phi^{\prime}(u)^{2}=1+\beta^{2} \operatorname{senh}^{2} \phi(u), \quad \phi^{\prime \prime}(u)=\beta^{2} \operatorname{senh} \phi(u) \cosh \phi(u) .
$$

Note que

$$
\frac{\partial x}{\partial u}=\left(\phi^{\prime}(u) \operatorname{senh} \phi(u), \phi^{\prime}(u) \cosh \phi(u) \cos \beta v, \phi^{\prime}(u) \cosh \phi(u) \operatorname{sen} \beta v, 0\right)
$$

$\mathrm{e}$

$$
\frac{\partial x}{\partial v}=(0,-\beta \operatorname{senh} \phi(u) \operatorname{sen} \beta v, \beta \operatorname{senh} \phi(u) \cos \beta v, 1) .
$$

Temos também que

$$
\left\langle\frac{\partial x}{\partial u}, \frac{\partial x}{\partial u}\right\rangle_{-1}=\phi^{\prime}(u)^{2}, \quad\left\langle\frac{\partial x}{\partial u}, \frac{\partial x}{\partial v}\right\rangle_{-1}=0, \quad\left\langle\frac{\partial x}{\partial v}, \frac{\partial x}{\partial v}\right\rangle_{-1}=1+\beta^{2} \operatorname{senh}^{2} \phi(u),
$$

logo a imersão $x$ é conforme.

Podemos assumir que $\phi(0)=0, \phi^{\prime}(u)>0$ e que $\phi$ está definida em um intervalo limitado.

O normal $\bar{N}$ a $\mathbb{H}^{2} \times \mathbb{R}$ em $\mathbb{L}^{4}$ é

$$
\bar{N}(u, v)=\left[\begin{array}{c}
\cosh \phi(u) \\
\operatorname{senh} \phi(u) \cos \beta v \\
\operatorname{senh} \phi(u) \operatorname{sen} \beta v \\
0
\end{array}\right] .
$$

Além disso, pelo que vimos na seção 3.2 , o normal $N$ a $\mathcal{H}_{\beta}$ em $\mathbb{S}^{2} \times \mathbb{R}$ é

$$
N(u, v)=\frac{1}{\phi^{\prime}(u)}\left[\begin{array}{c}
0 \\
\operatorname{sen} \beta v \\
-\cos \beta v \\
\beta \operatorname{senh} \phi(u)
\end{array}\right] .
$$

Vamos calcular o operador forma $A_{\mathcal{H}_{\beta}}$ de $\mathcal{H}_{\beta}$. Temos:

$$
\begin{gathered}
\frac{\partial^{2} x}{\partial u^{2}}=\left[\begin{array}{c}
\phi^{\prime \prime}(u) \operatorname{senh} \phi(u)-\phi^{\prime}(u)^{2} \cosh \phi(u) \\
\phi^{\prime \prime}(u) \cosh \phi(u) \cos \beta v-\phi^{\prime}(u)^{2} \operatorname{senh} \phi(u) \cos \beta v \\
\phi^{\prime \prime}(u) \cosh \phi(u) \cos \beta v-\phi^{\prime}(u)^{2} \operatorname{senh} \phi(u) \operatorname{sen} \beta v \\
0
\end{array}\right], \\
\frac{\partial^{2} x}{\partial v^{2}}=\left[\begin{array}{c}
0 \\
-\beta^{2} \operatorname{senh} \phi(u) \cos \beta v \\
-\beta^{2} \operatorname{senh} \phi(u) \operatorname{sen} \beta v \\
0
\end{array}\right]
\end{gathered}
$$




$$
\frac{\partial^{2} x}{\partial u \partial v}=\left[\begin{array}{c}
0 \\
-\beta \phi^{\prime}(u) \cosh \phi(u) \operatorname{sen} \beta v \\
\beta \phi^{\prime}(u) \cosh \phi(u) \cos \beta v \\
0
\end{array}\right]
$$

Portanto,

$$
\left\langle\frac{\partial^{2} x}{\partial u^{2}}, N\right\rangle_{-1}=0,\left\langle\frac{\partial^{2} x}{\partial v^{2}}, N\right\rangle_{-1}=0,\left\langle\frac{\partial^{2} x}{\partial u \partial v}, N\right\rangle_{-1}=-\beta \cosh \phi(u) .
$$

Daí, $A_{\mathcal{H}_{\beta}}$ é dado na base $\left\{\frac{\partial}{\partial u}, \frac{\partial}{\partial v}\right\}$ por

$$
\begin{aligned}
A_{\mathcal{H}_{\beta}} & =\left[\begin{array}{cc}
\frac{1}{\phi^{\prime}(u)^{2}} & 0 \\
0 & \frac{1}{\phi^{\prime}(u)^{2}}
\end{array}\right]\left[\begin{array}{cc}
0 & -\beta \cosh \phi(u) \\
-\beta \cos \phi(u) & 0
\end{array}\right] \\
& =-\frac{\beta \cosh \phi(u)}{\phi^{\prime}(u)^{2}}\left[\begin{array}{ll}
0 & 1 \\
1 & 0
\end{array}\right] .
\end{aligned}
$$

Além disso,

$$
\nu_{\mathcal{H}_{\beta}}=\left\langle\frac{\partial}{\partial t}, N\right\rangle_{-1}=\frac{\beta \operatorname{senh} \phi(u)}{\phi^{\prime}(u)}
$$

e

$$
\begin{aligned}
d x\left(T_{\mathcal{H}_{\beta}}\right) & =\frac{\partial}{\partial t}-\nu N \\
& =(0,0,0,1)-\frac{\beta \operatorname{senh} \phi(u)}{\phi^{\prime}(u)^{2}}(0, \operatorname{sen} \beta v,-\cos \beta v, \beta \operatorname{senh} \phi(u)) \\
& =\frac{1}{\phi^{\prime}(u)^{2}} \frac{\partial x}{\partial v}
\end{aligned}
$$

$\log 0$

$$
T_{\mathcal{H}_{\beta}}=\frac{1}{\phi^{\prime}(u)^{2}} \frac{\partial}{\partial v}
$$

Note que quando $\beta=0$, a fórmula define um plano vertical $\mathbb{H}^{1} \times \mathbb{R}$, e quando $\beta \rightarrow \infty$, a superfície converge para uma folheação por planos horizontais $\mathbb{H}^{2} \times\{t\}$.

Catenoides: São superfícies mínimas de rotação em $\mathbb{H}^{2} \times \mathbb{R}$. Para descrever os catenoides, vamos utilizar outro modelo para o espaço hiperbólico $\mathbb{H}^{2}$, o disco de Poincaré. Este modelo consiste no disco

$$
\mathbb{D}=\left\{(x, y) \in \mathbb{R}^{2} ; x^{2}+y^{2} \leq 1\right\}
$$

munido da métrica Riemanniana

$$
d s_{\mathbb{D}}^{2}=4 \frac{d x^{2}+d y^{2}}{\left(1-x^{2}-y^{2}\right)^{2}} .
$$

Para maiores detalhes sobre este e outros modelos do espaço hiperbólico, veja [CFK ${ }^{+}$97]. A isometria entre $\mathbb{D}$ e $\mathbb{H}^{2}$ é dada por

$$
(x, y) \mapsto\left(\frac{1+x^{2}+y^{2}}{1-x^{2}-y^{2}}, \frac{2 x}{1-x^{2}-y^{2}}, \frac{2 y}{1-x^{2}-y^{2}}\right),
$$


logo a isometria entre $\mathbb{D} \times \mathbb{R}$ e $\mathbb{H}^{2} \times \mathbb{R}$ é dada por

$$
(x, y, t) \mapsto\left(\frac{1+x^{2}+y^{2}}{1-x^{2}-y^{2}}, \frac{2 x}{1-x^{2}-y^{2}}, \frac{2 y}{1-x^{2}-y^{2}}, t\right) .
$$

Seja $\pi$ um plano geodésico vertical contendo a origem em $\mathbb{D} \times \mathbb{R}$. Seja $\gamma$ uma curva em $\pi$ e assuma que $\gamma$ seja gráfico sobre o eixo vertical. Considere a superfície de revolução $S$ obtida rotacionando $\gamma$ ao redor do eixo vertical. Quando $S$ for mínima, $S$ será chamada de catenoide.

A menos de uma rotação, podemos tomar o plano $\pi=\{(x, 0, t) \in \mathbb{D} \times \mathbb{R}\}$. Logo a curva $\gamma$ pode ser parametrizada por $\gamma(t)=(r(t), 0, t)$, onde $r(t)$ denota a distância euclidiana entre um ponto de $\gamma$ na altura $t$ e o eixo vertical, e portanto a superfície $S$ é parametrizada por

$$
\bar{x}(t, \theta)=(r(t) \cos \theta, r(t) \operatorname{sen} \theta, t) .
$$

Proposição 3.4.1. Seja $\bar{x}$ parametrização de uma superfície $S \in \mathbb{D} \times \mathbb{R}$ de rotação como descrita acima. Suponha que $\bar{x}$ seja conforme. Então $S$ é um catenoide.

Demonstração. Temos que a parametrização $\bar{x}$ é dada por

$$
\bar{x}(t, \theta)=(r(t) \cos \theta, r(t) \operatorname{sen} \theta, t) .
$$

Além disso,

$$
d s_{\mathbb{D}}^{2}\left(\frac{\partial \bar{x}}{\partial t}, \frac{\partial \bar{x}}{\partial t}\right)=4 \frac{r^{\prime}(t)^{2}}{\left(1-r(t)^{2}\right)^{2}}+1
$$

e

$$
s_{\mathbb{D}}^{2}\left(\frac{\partial \bar{x}}{\partial \theta}, \frac{\partial \bar{x}}{\partial \theta}\right)=4 \frac{r(t)^{2}}{\left(1-r(t)^{2}\right)^{2}} .
$$

Como $\bar{x}$ é conforme, segue que

$$
\begin{aligned}
& 4 \frac{r^{\prime}(t)^{2}}{\left(1-r(t)^{2}\right)^{2}}+1=4 \frac{r(t)^{2}}{\left(1-r(t)^{2}\right)^{2}} \\
\Rightarrow \quad & r^{\prime}(t)^{2}=\frac{3}{2} r(t)^{2}-\frac{1+r(t)^{4}}{4} .
\end{aligned}
$$

Logo, como pode ser visto em [NR02], $r(t)$ é solução da EDO

$$
4 r(t) r^{\prime \prime}(t)-4 r^{\prime}(t)^{2}-\left(1-r(t)^{4}\right)=0,
$$

e portanto $S$ é mínima.

Passando $S$ para $\mathbb{H}^{2} \times \mathbb{R}$ através da isometria dada acima, podemos parametrizar $S$ em $\mathbb{H}^{2} \times \mathbb{R}$ por

$$
x(t, \theta)=\left(\frac{1+r(t)^{2}}{1-r(t)^{2}}, \frac{2 r(t)}{1-r(t)^{2}} \cos \theta, \frac{2 r(t)}{1-r(t)^{2}} \operatorname{sen} \theta, t\right) .
$$

Seja $R(t)=\operatorname{arccosh}\left(\frac{1+r(t)^{2}}{1-r(t)^{2}}\right)$. Como $\cosh ^{2} R(T)-\operatorname{senh}^{2} R(t)=1$, temos que

$$
\operatorname{senh}^{2} R(t)=\left(\frac{1+r(t)^{2}}{1-r(t)^{2}}\right)^{2}-1=\left(\frac{2 r(t)}{1-r(t)^{2}}\right)
$$

e portanto a parametrização $x$ pode ser escrita como

$$
x(t, \theta)=(\cosh R(t), \operatorname{senh} R(t) \cos \theta, \operatorname{senh} R(t) \operatorname{sen} \theta, t) .
$$

Para $\alpha \neq 0$, seja o catenoide $\mathcal{C}_{\alpha}$ dado pela seguinte imersão: 


$$
x(u, v)=\left[\begin{array}{c}
\cosh \psi(u) \\
\operatorname{senh} \psi(u) \cos \alpha v \\
\operatorname{senh} \psi(u) \operatorname{sen} \alpha v \\
u
\end{array}\right]
$$

onde a função $\psi$ satisfaz

$$
1+\psi^{\prime}(u)^{2}=\alpha^{2} \operatorname{senh}^{2} \psi(u), \quad \psi^{\prime \prime}(u)=\alpha^{2} \operatorname{senh} \psi(u) \cosh \psi(u) .
$$

Podemos assumir que $\psi^{\prime}(0)=0$ e $\psi(u)>0$. Como $\psi^{\prime \prime}(u)>0$, temos que $\psi^{\prime}$ é crescente. Agora, como $\psi^{\prime}(0)=0$, temos que, para todo $u>0, \psi^{\prime}(u)>0$, logo $\psi$ é crescente neste intervalo. Concluímos entao que, para $u>0, \psi$ possui inversa $\psi^{-1}$. Então temos que

$$
\frac{d \psi^{-1}}{d \psi}=\frac{1}{\psi^{\prime}}
$$

e portanto

$$
u=\int_{0}^{u} d \psi^{-1}=\int_{\psi(0)}^{\psi(u)} \frac{d \psi^{-1}}{d \psi} d \psi=\int_{\psi(0)}^{\psi(u)} \frac{1}{\psi^{\prime}} d \psi
$$

Desta forma também podemos assumir que $\psi$ está definida em um intervalo $\left(-u_{0}, u_{0}\right)$ com

$$
u_{0}=\int_{\psi(0)}^{\infty} \frac{d \psi}{\sqrt{\alpha^{2} \operatorname{senh}^{2} \psi-1}}=\int_{1}^{\infty} \frac{d x}{\sqrt{\left(x^{2}+\alpha^{2}\right)\left(x^{2}-1\right)}}
$$

Daí temos

$$
u_{0} \leq \int_{1}^{\infty} \frac{d x}{x \sqrt{x^{2}-1}}=\frac{\pi}{2} .
$$

Desta forma temos que o catenoide $\mathcal{C}_{\alpha}$ tem altura menor que $\pi$, e esta tende a 0 quando $\alpha \rightarrow \infty$ e tende a $\pi$ quando $\alpha \rightarrow 0$.

Note que

$$
\frac{\partial x}{\partial u}=\left(\psi^{\prime}(u) \operatorname{senh} \psi(u), \psi^{\prime}(u) \cosh \psi(u) \cos \alpha v, \psi^{\prime}(u) \cosh \psi(u) \operatorname{sen} \alpha v, 1\right)
$$

e

$$
\frac{\partial x}{\partial v}=(0,-\alpha \operatorname{senh} \psi(u) \operatorname{sen} \alpha v, \alpha \operatorname{senh} \psi(u) \cos \alpha v, 0)
$$

Temos também que

$$
\left\langle\frac{\partial x}{\partial u}, \frac{\partial x}{\partial u}\right\rangle_{-1}=1+\psi^{\prime}(u)^{2}, \quad\left\langle\frac{\partial x}{\partial u}, \frac{\partial x}{\partial v}\right\rangle_{-1}=0, \quad\left\langle\frac{\partial x}{\partial v}, \frac{\partial x}{\partial v}\right\rangle_{-1}=\alpha^{2} \operatorname{senh}^{2} \psi(u),
$$

logo a imersão $x$ é conforme.

O normal $\bar{N}$ a $\mathbb{H}^{2} \times \mathbb{R}$ em $\mathbb{L}^{4}$ é

$$
\bar{N}(u, v)=\left[\begin{array}{c}
\cosh \psi(u) \\
\operatorname{senh} \psi(u) \cos \alpha v \\
\operatorname{senh} \psi(u) \operatorname{sen} \alpha v \\
0
\end{array}\right]
$$

Além disso, pelo que vimos na seção 3.2 , o normal $N$ a $\mathcal{C}_{\alpha}$ em $\mathbb{H}^{2} \times \mathbb{R}$ é

$$
N(u, v)=\frac{1}{\alpha \operatorname{senh} \psi(u)}\left[\begin{array}{c}
-\operatorname{senh} \psi(u) \\
-\cosh \psi(u) \cos \alpha v \\
-\cosh \psi(u) \operatorname{sen} \alpha v \\
\psi^{\prime}(u)
\end{array}\right] .
$$


Vamos calcular o operador forma $A_{\mathcal{C}_{\alpha}}$ de $\mathcal{C}_{\alpha}$. Temos:

$$
\begin{gathered}
\frac{\partial^{2} x}{\partial u^{2}}=\left[\begin{array}{c}
\psi^{\prime \prime}(u) \operatorname{senh} \psi(u)-\psi^{\prime}(u)^{2} \cosh \psi(u) \\
\psi^{\prime \prime}(u) \cosh \psi(u) \cos \alpha v-\psi^{\prime}(u)^{2} \operatorname{senh} \psi(u) \cos \alpha v \\
\psi^{\prime \prime}(u) \cosh \psi(u) \operatorname{sen} \alpha v-\psi^{\prime}(u)^{2} \operatorname{senh} \psi(u) \operatorname{sen} \alpha v \\
0
\end{array}\right], \\
\frac{\partial^{2} x}{\partial v^{2}}=\left[\begin{array}{c}
0 \\
-\alpha^{2} \operatorname{senh} \psi(u) \cos \alpha v \\
-\alpha^{2} \operatorname{senh} \psi(u) \operatorname{sen} \alpha v \\
0
\end{array}\right], \\
\frac{\partial^{2} x}{\partial u \partial v}=\left[\begin{array}{c}
\alpha \\
-\alpha \psi^{\prime}(u) \cosh \psi(u) \operatorname{sen} \alpha v \\
\alpha \psi^{\prime}(u) \cosh \psi(u) \cos \alpha v \\
0
\end{array}\right]
\end{gathered}
$$

Portanto,

$$
\left\langle\frac{\partial^{2} x}{\partial u^{2}}, N\right\rangle_{-1}=-\alpha \cosh \psi(u),\left\langle\frac{\partial^{2} x}{\partial v^{2}}, N\right\rangle_{-1}=\alpha \cosh \psi(u),\left\langle\frac{\partial^{2} x}{\partial u \partial v}, N\right\rangle_{-1}=0 .
$$

Daí, $A_{\mathcal{C}_{\alpha}}$ é dado na base $\left\{\frac{\partial}{\partial u}, \frac{\partial}{\partial v}\right\}$ por

$$
\begin{aligned}
A_{\mathcal{C}_{\alpha}} & =\left[\begin{array}{cc}
\frac{1}{1+\psi^{\prime}(u)^{2}} & 0 \\
0 & \frac{1}{1+\psi^{\prime}(u)^{2}}
\end{array}\right]\left[\begin{array}{cc}
-\alpha \cosh \psi(u) & 0 \\
0 & \alpha \cosh \psi(u)
\end{array}\right] \\
& =-\frac{\alpha \cosh \psi(u)}{1+\psi^{\prime}(u)^{2}}\left[\begin{array}{cc}
1 & 0 \\
0 & -1
\end{array}\right] .
\end{aligned}
$$

Além disso,

$$
\nu_{\mathcal{C}_{\alpha}}=\left\langle\frac{\partial}{\partial t}, N\right\rangle=\frac{\psi^{\prime}(u)}{\alpha \operatorname{senh} \psi(u)}
$$

e

$$
\begin{aligned}
d x\left(T_{\mathcal{C}_{\alpha}}\right) & =\frac{\partial}{\partial t}-\nu N \\
& =(0,0,0,1)-\frac{\psi^{\prime}(u)}{\alpha^{2} \operatorname{senh} \psi(u)^{2}}\left(-\operatorname{senh} \psi(u),-\cosh \psi(u) \cos \alpha v,-\cosh \psi(u) \operatorname{sen} \alpha v, \psi^{\prime}(u)\right) \\
& =\frac{1}{1+\psi^{\prime}(u)^{2}} \frac{\partial x}{\partial v}
\end{aligned}
$$

$\log \mathrm{O}$

$$
T_{\mathcal{C}_{\alpha}}=\frac{1}{1+\psi^{\prime}(u)^{2}} \frac{\partial}{\partial v}
$$

Proposição 3.4.2. A superfície conjugada do catenoide $\mathcal{C}_{\alpha}$ é o helicoide $\mathcal{H}_{\beta}$ tal que $\beta^{2}=1+\alpha^{2}$ e $\alpha$ e $\beta$ com mesmo sinal. 
Demonstração. Sejam $y_{1}(u)=\alpha \cosh \psi(u)$ e $y_{2}(u)=\beta \cosh \phi(u)$. Note que

$$
\begin{aligned}
\left(y_{1}(u)^{2}-\alpha^{2}\right)\left(y_{1}(u)^{2}-\beta^{2}\right) & =\left(\alpha^{2} \cosh ^{2} \psi(u)-\alpha^{2}\right)\left(\alpha^{2} \cosh ^{2} \psi(u)-\alpha^{2}-1\right) \\
& =\left(\alpha^{2} \operatorname{senh}^{2} \psi(u)\right)\left(\alpha^{2} \operatorname{senh}^{2} \psi(u)-1\right) \\
& =\left(\alpha^{2} \operatorname{sen}^{2} \psi(u)\right)\left(\psi^{\prime}(u)^{2}\right) \\
& =y_{1}^{\prime}(u)^{2} .
\end{aligned}
$$

Da mesma forma temos

$$
\left(y_{2}(u)^{2}-\alpha^{2}\right)\left(y_{2}(u)^{2}-\beta^{2}\right)=y_{2}^{\prime}(u)^{2} .
$$

Portanto $y_{1}$ e $y_{2}$ são soluções da equação (3.20):

$$
\left(y^{\prime}\right)^{2}=\left(y^{2}-\alpha^{2}\right)\left(y^{2}-\beta^{2}\right),
$$

e (3.21):

$$
y^{\prime \prime}=y\left(2 y^{2}-\alpha^{2}-\beta^{2}\right) .
$$

Como

$$
1+\psi^{\prime}(u)^{2}=\alpha^{2} \operatorname{senh}^{2} \psi(u)=y_{1}(u)^{2}-\alpha^{2},
$$

usando o fato de que $\psi^{\prime}(0)=0$ e $\alpha^{2}=\beta^{2}-1$, temos

$$
y_{1}(0)^{2}=\beta^{2} .
$$

Substituindo em (3.20) temos

$$
y_{1}^{\prime}(0)=0 .
$$

Além disso, como $\phi(0)=0$, temos

$$
y_{2}(0)=\beta \cosh \phi(0)=\beta \text {. }
$$

Substituindo em (3.20) temos

$$
y_{2}^{\prime}(0)=0 .
$$

Sabemos que $\cosh \psi(0)>0, \operatorname{logo} y_{1}(0)$ tem o mesmo sinal de $\alpha$, e como $\alpha$ e $\beta$ têm o mesmo sinal, por (3.25) e (3.26) segue que $y_{1}(0)=y_{2}(0)$. Pelo teorema de existência e unicidade de soluções de EDO concluímos que $y_{1}=y_{2}$.

Precisamos mostrar que $\mathcal{C}_{\alpha}$ e $\mathcal{H}_{\beta}$ são localmente isométricos, $A_{\mathcal{H}_{\beta}}=J A_{\mathcal{C}_{\alpha}}, T_{\mathcal{H}_{\beta}}=J T_{\mathcal{C}_{\alpha}}$ e $\nu_{\mathcal{H}}{ }_{\beta}=\nu_{\mathcal{C}_{\alpha}}$.

1. Como

$$
\begin{aligned}
\phi^{\prime}(u) & =1+\beta^{2} \operatorname{senh}^{2} \phi(u)=1+\beta^{2} \cosh ^{2} \phi(u)-\beta^{2}=-\alpha^{2}+y_{2}(u)^{2}=-\alpha^{2}+y_{1}(u)^{2} \\
& =-\alpha^{2}+\alpha^{2} \cosh ^{2} \psi(u)=\alpha^{2} \operatorname{senh}^{2} \psi(u)=1+\psi^{\prime}(u)^{2}
\end{aligned}
$$

segue que $\mathcal{C}_{\alpha}$ e $\mathcal{H}_{\beta}$ são localmente isométricos;

2. Utilizando o item anterior, como $\beta \cosh \phi(u)=y_{1}(u)=y_{2}(u)=\alpha \cosh \psi(u)$,

$$
\begin{aligned}
A_{\mathcal{H}_{\beta}} & =-\frac{\beta \cosh \phi(u)}{\phi^{\prime}(u)^{2}}\left[\begin{array}{ll}
0 & 1 \\
1 & 0
\end{array}\right]=-\frac{\alpha \cosh \psi(u)}{1+\psi^{\prime}(U)^{2}}\left[\begin{array}{ll}
0 & 1 \\
1 & 0
\end{array}\right]=-\frac{\alpha \cosh \psi(u)}{1+\psi^{\prime}(U)^{2}}\left[\begin{array}{cc}
0 & -1 \\
1 & 0
\end{array}\right]\left[\begin{array}{cc}
1 & 0 \\
0 & -1
\end{array}\right] \\
& =J A_{\mathcal{C}_{\alpha}}
\end{aligned}
$$

3.

$$
T_{\mathcal{H}_{\beta}}=\frac{1}{\phi^{\prime}(u)^{2}} \frac{\partial}{\partial v}=\frac{1}{1+\psi^{\prime}(u)^{2}} J \frac{\partial}{\partial u}=J T_{\mathcal{C}_{\alpha}} ;
$$


4. Temos

$$
\nu_{\mathcal{C}_{\alpha}}=\frac{\psi^{\prime}(u)}{\alpha \operatorname{sen} \psi(u)}=\frac{y_{1}^{\prime}(u)}{\alpha^{2} \operatorname{senh}^{2} \psi(u)}=\frac{y_{1}^{\prime}(u)}{\alpha^{2} \cosh ^{2} \psi(u)-\alpha^{2}}=\frac{y_{1}^{\prime}(u)}{y_{1}(u)^{2}-\alpha^{2}}
$$

e

$$
\begin{aligned}
\nu_{\mathcal{H}} & =\frac{\beta \operatorname{senh} \phi(u)}{\phi^{\prime}(u)}=\frac{\beta \phi^{\prime}(u) \operatorname{senh} \phi(u)}{\phi^{\prime}(u)^{2}}=\frac{y_{2}^{\prime}(u)}{1+\beta^{2} \operatorname{senh}^{2} \phi(u)}=\frac{y_{2}^{\prime}(u)}{1-\beta^{2}+\beta^{2} \cosh ^{2} \phi(u)} \\
& =\frac{y_{2}^{\prime}(u)}{y_{2}(u)^{2}-\alpha^{2}},
\end{aligned}
$$

$\log 0 \nu_{\mathcal{H}_{\beta}}=\nu_{\mathcal{C}_{\alpha}}$.

Portanto segue que $\mathcal{H}_{\beta}$ é superfície conjugada de $\mathcal{C}_{\alpha}$, como queríamos.

Superfícies mínimas folheadas por horocírculos: Círculos generalizados (ou apenas círculos) em $\mathbb{H}^{2}$ são dados pela interseç̧ão de $\mathbb{H}^{2}$ com planos de $\mathbb{L}^{3}$ que não contém a origem. Como quaisquer três pontos não colineares de $\mathbb{H}^{2}$ determinam um plano de $\mathbb{L}^{3}$, estes também determinam um círculo de $\mathbb{H}^{2}$. Estes planos são dados pelos pontos $x \in \mathbb{H}^{2}$ tais que

$$
\langle v, x\rangle_{\varepsilon}=-v_{0} x_{0}+v_{1} x_{1}+v_{2} x_{2}=-\lambda,
$$

para algum $v \in \mathbb{L}^{3}$ fixado e um número real $\lambda$, com $v$ e $\lambda$ não nulos. Os círculos tais que $\langle v, v\rangle_{-1}=0$ são chamados de horocírculos.

Vamos determinar as superfícies folheadas por horocírculos com mesmo ponto assintótico, ou seja, dadas pelo mesmo vetor $v \in \mathbb{L}^{3}$. Dado $\lambda \in \mathbb{R}$, tome o horocírculo de equação $-v_{0} x_{0}+v_{1} x_{1}+$ $v_{2} x_{2}=-\lambda$. Como $v_{0}^{2}=v_{1}^{2}+v_{2}^{2}$, podemos supor que $v_{0}=1$, e a menos de uma isometria de $\mathbb{H}^{2}$, mais precisamente uma rotação, podemos supor que $v_{1}=0$ e $v_{2}=1$. Portanto, a menos de uma isometria, o horocírculo é dado pela equação

$$
-x_{0}+x_{2}=-\lambda
$$

Além disso devemos ter $\lambda>0$. Sabemos que $-x_{0}^{2}+x_{1}^{2}+x_{2}^{2}=-1$, logo

$$
\begin{aligned}
& -x_{0}^{2}+x_{2}^{2}=-1-x_{1}^{2} \\
\Rightarrow & \left(x_{2}-x_{0}\right)\left(x_{2}+x_{0}\right)=-1-x_{1}^{2} \\
\Rightarrow \quad & -\lambda\left(x_{2}+x_{0}\right)=-1-x_{1}^{2} \\
\Rightarrow & x_{2}+x_{0}=\frac{-1-x_{1}^{2}}{\lambda} .
\end{aligned}
$$

Portanto

$$
x_{0}=\frac{\lambda}{2}+\frac{1+x_{1}^{2}}{2 \lambda}
$$

$\mathrm{e}$

$$
x_{2}=-\frac{\lambda}{2}+\frac{1+x_{1}^{2}}{2 \lambda}
$$

Desta forma, as superfícies que procuramos podem ser parametrizadas por

$$
x(u, v)=\left[\begin{array}{c}
\frac{\lambda(u)}{2}+\frac{1+f(u, v)^{2}}{2 \lambda} \\
f(u, v) \\
-\frac{\lambda(u)}{2}+\frac{1+f(u, v)^{2}}{2 \lambda} \\
u
\end{array}\right],
$$

com $\lambda>0$, e como $f$ não pode depender apenas de $u$, podemos supor que $f_{v}>0$. 
Note que

$$
\frac{\partial x}{\partial u}=\left(\frac{\lambda^{\prime}}{2}+\frac{\lambda^{\prime}\left(f^{2}-1\right)}{2 \lambda^{2}}, f_{u},-\frac{\lambda^{\prime}}{2}+\frac{\lambda^{\prime}\left(f^{2}-1\right)}{2 \lambda^{2}}, 1\right)
$$

$$
\frac{\partial x}{\partial v}=\left(\frac{f f_{v}}{\lambda}, f_{v}, \frac{f f_{v}}{\lambda}, 0\right)
$$

Temos também que

$$
\left\langle\frac{\partial x}{\partial u}, \frac{\partial x}{\partial u}\right\rangle_{-1}=-\frac{\left(\lambda^{\prime}\right)^{2}\left(f^{2}-1\right)}{\lambda^{2}}+f_{u}^{2}+1, \quad\left\langle\frac{\partial x}{\partial u}, \frac{\partial x}{\partial v}\right\rangle_{-1}=-\frac{\lambda^{\prime} f f_{v}}{\lambda}+f_{u} f_{v}, \quad\left\langle\frac{\partial x}{\partial v}, \frac{\partial x}{\partial v}\right\rangle_{-1}=f_{v}^{2} .
$$

Vamos supor que $x$ é conforme e mínima. Temos que $x$ é conforme se e somente se

$$
-\frac{\lambda^{\prime} f f_{v}}{\lambda}+f_{u} f_{v}=0 \Rightarrow f_{u}=\frac{f \lambda^{\prime}}{\lambda}
$$

e

$$
-\frac{\left(\lambda^{\prime}\right)^{2}\left(f^{2}-1\right)}{\lambda^{2}}+f_{u}^{2}+1=f_{v}^{2} \Rightarrow f_{v}^{2}=\left(\frac{\lambda^{\prime}}{\lambda}\right)^{2}+1 .
$$

Derivando a equação (3.28) em $v$, como $f_{v}>0$ temos

$$
2 f_{v} f_{v v}=0 \Rightarrow f_{v v}=0
$$

portanto

$$
f(u, v)=\alpha(u) v+\beta(u) .
$$

Com isso, utilizando (3.27), temos

$$
f_{u}(u, v)=\alpha^{\prime}(u) v+\beta^{\prime}(u)=(\alpha(u) v+\beta(u)) \frac{\lambda^{\prime}(u)}{\lambda(u)},
$$

$\operatorname{logo} \alpha^{\prime}=\alpha \frac{\lambda^{\prime}}{\lambda}$ e $\beta^{\prime}=\beta \frac{\lambda^{\prime}}{\lambda}$.

Como $x$ é mínima, pelo que vimos na seção $3.2, \Delta x$ é proporcional à $\bar{N}$, o normal a $\mathbb{H}^{2} \times \mathbb{R}$ em $\mathbb{E}^{4}$ dado por

$$
\bar{N}=\left[\begin{array}{c}
\frac{\lambda(u)}{2}+\frac{1+f(u, v)^{2}}{2 \lambda} \\
f(u, v) \\
-\frac{\lambda(u)}{2}+\frac{1+f(u, v)^{2}}{2 \lambda} \\
0
\end{array}\right],
$$

ou seja, $\Delta x=\mu \bar{N}$, para alguma função $\mu$.

Note que

$$
\frac{\partial^{2} x}{\partial u^{2}}=\left[\begin{array}{c}
\frac{\lambda^{\prime \prime}}{2}+\frac{\left(\lambda^{\prime}\right)^{2}}{\lambda^{3}}+\frac{\lambda^{\prime \prime}\left(f^{2}-1\right)}{2 \lambda^{2}} \\
\frac{\lambda^{\prime \prime} f}{\lambda} \\
-\frac{\lambda^{\prime \prime}}{2}+\frac{\left(\lambda^{\prime}\right)^{2}}{\lambda^{3}}+\frac{\lambda^{\prime \prime}\left(f^{2}-1\right)}{2 \lambda^{2}} \\
0
\end{array}\right]
$$

e

$$
\frac{\partial^{2} x}{\partial v^{2}}=\left[\begin{array}{c}
\frac{1}{\lambda}+\frac{\left(\lambda^{\prime \prime}\right)^{2}}{\lambda^{3}} \\
0 \\
\frac{1}{\lambda}+\frac{\left(\lambda^{\prime \prime}\right)^{2}}{\lambda^{3}} \\
0
\end{array}\right]
$$

A segunda coordenada de $\Delta x=\frac{\partial^{2} x}{\partial u^{2}}+\frac{\partial^{2} x}{\partial v^{2}}=\mu \bar{N}$ nos da $\mu=\frac{\lambda^{\prime \prime}}{\lambda}$, e a primeira coordenada nos 
dá

$$
\begin{aligned}
& \frac{\lambda^{\prime \prime}}{2}+\frac{\left(\lambda^{\prime}\right)^{2}}{\lambda^{3}}+\frac{\lambda^{\prime \prime}\left(f^{2}-1\right)}{2 \lambda^{2}}+\frac{1}{\lambda}+\frac{\left(\lambda^{\prime \prime}\right)^{2}}{\lambda^{3}}=\frac{\lambda^{\prime \prime}}{2}+\frac{\lambda^{\prime \prime}\left(f^{2}+1\right)}{2 \lambda^{2}} \\
\Rightarrow & -\frac{\lambda^{\prime \prime}}{\lambda^{2}}+\frac{2\left(\lambda^{\prime}\right)^{2}}{\lambda^{3}}=-\frac{1}{\lambda} \\
\Rightarrow & \left(-\frac{\lambda^{\prime}}{\lambda^{2}}\right)^{\prime}=-\frac{1}{\lambda} \\
\Rightarrow & \left(\frac{1}{\lambda}\right)^{\prime \prime}=\frac{1}{\lambda} .
\end{aligned}
$$

Sabemos que as soluções de (3.29) são da forma $\lambda(u)=c_{1} \cos u+c_{2} \operatorname{sen} u$, onde $c_{1}$ e $c_{2}$ são constantes. Então podemos escolher $\lambda(u)=\alpha(u)=\frac{1}{\cos u}$ e $\beta(u)=0$, para $u \in\left(-\frac{\pi}{2}, \frac{\pi}{2}\right)$. Desta forma, a aplicação

$$
x(u, v)=\left[\begin{array}{c}
\frac{v^{2}+1}{2 \cos u}+\frac{\cos u}{2} \\
\frac{v}{\cos u} \\
\frac{v^{2}-1}{2 \cos u}+\frac{\cos u}{2} \\
u
\end{array}\right],
$$

definida para $(u, v) \in\left(-\frac{\pi}{2}, \frac{\pi}{2}\right) \times \mathbb{R}$ é um mergulho mínimo conforme tal que as curvas $u=u_{0}$ são horocírculos em $\mathbb{H}^{2}$ com mesmo ponto assintótico. Denotaremos esta superfície por $\mathcal{C}_{0}$. Pela seção 3.2 , o vetor normal $N$ normal à $\mathcal{C}_{0}$ em $\mathbb{H}^{2} \times \mathbb{R}$ é dado por

$$
N(u, v)=\left[\begin{array}{c}
-\frac{v^{2}+1}{2}+\frac{\cos ^{2} u}{2} \\
-v \\
\frac{1-v^{2}}{2}+\frac{\cos ^{2} u}{2} \\
\operatorname{sen} u
\end{array}\right] .
$$

Note que

$$
\frac{\partial x}{\partial u}=\left(\frac{\operatorname{sen} u\left(v^{2}+1\right)}{2 \cos ^{2} u}-\frac{\operatorname{sen} u}{2}, \frac{\operatorname{sen} u}{\cos ^{2} u} v, \frac{\operatorname{sen} u\left(v^{2}-1\right)}{2 \cos ^{2} u}-\frac{\operatorname{sen} u}{2}, 1\right)
$$

$\mathrm{e}$

$$
\frac{\partial x}{\partial v}=\left(\frac{v}{\cos u}, \frac{1}{\cos u}, \frac{v}{\cos u}, 0\right) .
$$

Temos também que

$$
\left\langle\frac{\partial x}{\partial u}, \frac{\partial x}{\partial u}\right\rangle_{-1}=\left\langle\frac{\partial x}{\partial v}, \frac{\partial x}{\partial v}\right\rangle_{-1}=\frac{1}{\cos ^{2} u}
$$

$\mathrm{e}$

$$
\left\langle\frac{\partial x}{\partial u}, \frac{\partial x}{\partial v}\right\rangle_{-1}=0 .
$$

Além disso,

$$
\begin{gathered}
\frac{\partial^{2} x}{\partial u^{2}}=\left[\begin{array}{c}
\frac{\operatorname{sen}^{2} u+1}{2 \cos ^{3} u}+\frac{\operatorname{sen}^{2} u}{\cos u}+\frac{\left(\operatorname{sen}^{2} u+1\right)\left(v^{2}-1\right)}{2 \cos u} \\
\frac{\operatorname{sen}^{2} u+1}{\cos ^{3} u} v \\
-\frac{\operatorname{sen}^{2} u+1}{2 \cos ^{3} u}++\frac{\operatorname{sen}^{2} u}{\cos u}+\frac{\left(\operatorname{sen}^{2} u+1\right)\left(v^{2}-1\right)}{2 \cos u} \\
0
\end{array}\right], \\
\frac{\partial^{2} x}{\partial v^{2}}=\left[\begin{array}{c}
\frac{1}{\cos u} \\
0 \\
\frac{1}{\cos u} \\
0
\end{array}\right],
\end{gathered}
$$




$$
\frac{\partial^{2} x}{\partial u \partial v}=\left[\begin{array}{c}
\frac{\operatorname{sen} u}{\cos 2} v \\
\frac{\operatorname{sen} u}{\cos ^{2} u} \\
\frac{\operatorname{sen}^{2} v}{\cos ^{2} v} \\
0
\end{array}\right] .
$$

Portanto,

$$
\left\langle\frac{\partial^{2} x}{\partial u^{2}}, N\right\rangle_{-1}=-\frac{1}{\cos u},\left\langle\frac{\partial^{2} x}{\partial v^{2}}, N\right\rangle_{-1}=\frac{1}{\cos u},\left\langle\frac{\partial^{2} x}{\partial u \partial v}, N\right\rangle_{-1}=0 .
$$

Daí, $A_{\mathcal{C}_{\alpha}}$ é dado na base $\left\{\frac{\partial}{\partial u}, \frac{\partial}{\partial v}\right\}$ por

$$
\begin{aligned}
A_{\mathcal{C}_{0}} & =\left[\begin{array}{cc}
\cos ^{2} u & 0 \\
0 & \cos ^{2} u
\end{array}\right]\left[\begin{array}{cc}
-\frac{1}{\cos u} & 0 \\
0 & \frac{1}{\cos u}
\end{array}\right] \\
& =-\cos u\left[\begin{array}{cc}
1 & 0 \\
0 & -1
\end{array}\right] .
\end{aligned}
$$

Além disso,

$$
\nu_{\mathcal{C}_{0}}=\left\langle\frac{\partial}{\partial t}, N\right\rangle_{-1}=\operatorname{sen} u
$$

$\mathrm{e}$

$$
\begin{aligned}
d x\left(T_{\mathcal{C}_{0}}\right) & =\frac{\partial}{\partial t}-\nu N \\
& =\left(\frac{\operatorname{sen} u\left(v^{2}+1\right)}{2}-\frac{\operatorname{sen} u \cos ^{2} u}{2}, v \operatorname{sen} u, \frac{\operatorname{sen} u\left(v^{2}-1\right)}{2}-\frac{\operatorname{sen} u \cos ^{2} u}{2}, \cos ^{2} u\right) \\
& =\cos ^{2} u \frac{\partial x}{\partial u},
\end{aligned}
$$

$\log 0$

$$
T_{\mathcal{C}_{0}}=\cos ^{2} u \frac{\partial}{\partial u} .
$$

Proposição 3.4.3. A superfície conjugada da superfície $\mathcal{C}_{0}$ é o helicoide $\mathcal{H}_{1}$.

Demonstração. No caso em que $\beta=1$, temos que $\phi^{\prime}(u)^{2}=1+\operatorname{senh}^{2} \phi(u)=\cosh ^{2} \phi(u)$. Como $\phi^{\prime}(u)>0$, segue que $\phi^{\prime}(u)=\cosh \phi(u)$. Resolvendo esta equação diferencial com valor inicial $\phi(0)=0$ obtemos a solução

$$
\phi(u)=\ln \left(\operatorname{tg}\left(\frac{u}{2}+\frac{\pi}{4}\right)\right) .
$$

Como

$$
\begin{aligned}
\cosh \phi(u) & =\frac{e^{\ln \left(\operatorname{tg}\left(\frac{u}{2}+\frac{\pi}{4}\right)\right)}+e^{-\ln \left(\operatorname{tg}\left(\frac{u}{2}+\frac{\pi}{4}\right)\right)}}{2}=\frac{\operatorname{tg}\left(\frac{u}{2}+\frac{\pi}{4}\right)+\frac{1}{\operatorname{tg}\left(\frac{u}{2}+\frac{\pi}{4}\right)}}{2} \\
& =\frac{1+\operatorname{tg}^{2}\left(\frac{u}{2}\right)}{1-\operatorname{tg}^{2}\left(\frac{u}{2}\right)}=\frac{1}{\cos ^{2}\left(\frac{u}{2}\right)-\operatorname{sen}^{2}\left(\frac{u}{2}\right)}=\frac{1}{\cos u},
\end{aligned}
$$

temos

$$
\phi^{\prime}(u)=\frac{1}{\cos u} .
$$


Além disso,

$$
\begin{aligned}
\operatorname{senh} \phi(u) & =\frac{e^{\ln \left(\operatorname{tg}\left(\frac{u}{2}+\frac{\pi}{4}\right)\right)}-e^{-\ln \left(\operatorname{tg}\left(\frac{u}{2}+\frac{\pi}{4}\right)\right)}}{2} \\
& =\frac{2 \operatorname{tg}\left(\frac{u}{2}\right)}{1-\operatorname{tg}^{2}\left(\frac{u}{2}\right)} \\
& =\operatorname{tg} u .
\end{aligned}
$$

Observe agora que:

1. Por (3.30) temos $\phi^{\prime}(u)^{2}=\frac{1}{\cos ^{2} u}$, $\log \mathrm{C} \mathcal{C}_{0}$ e $\mathcal{H}_{1}$ são localmente isométricos.

2. Pelo item anterior, temos

$$
\begin{aligned}
A_{\mathcal{H}_{1}} & =-\frac{\cosh \phi(u)}{\phi^{\prime}(u)^{2}}\left[\begin{array}{ll}
0 & 1 \\
1 & 0
\end{array}\right]=-\cos u\left[\begin{array}{ll}
0 & 1 \\
1 & 0
\end{array}\right]=-\cos u\left[\begin{array}{cc}
0 & -1 \\
1 & 0
\end{array}\right]\left[\begin{array}{cc}
1 & 0 \\
0 & -1
\end{array}\right] \\
& =J A_{\mathcal{C}_{0}}
\end{aligned}
$$

3.

$$
T_{\mathcal{H}_{1}}=\frac{1}{\phi^{\prime}(u)^{2}} \frac{\partial}{\partial v}=\cos ^{2} u J \frac{\partial}{\partial u}=J T_{\mathcal{C}_{0}}
$$

4. Por (3.31) temos

$$
\nu_{\mathcal{H}_{1}}=\frac{\operatorname{senh} \phi(u)}{\phi^{\prime}(u)}=\operatorname{sen} u=\nu_{\mathcal{C}_{0}}
$$

Portanto segue que $\mathcal{H}_{1}$ é superfície conjugada de $\mathcal{C}_{0}$, como queríamos.

Superfícies mínimas folheadas por equidistantes: Curvas equidistantes em $\mathbb{H}^{2}$ são curvas cujos pontos equidistam de uma determinada geodésica de $\mathbb{H}^{2}$. Para $\gamma \in(0,1)$ ou $\gamma \in(-1,0)$, considere superfície $\mathcal{G}_{\gamma}$, que chamaremos de catenoide generalizado, dada pela seguinte imersão:

$$
x(u, v)=\left[\begin{array}{c}
\cosh \chi(u) \cosh \gamma v \\
\operatorname{senh} \chi(u) \\
\cosh \chi(u) \operatorname{senh} \gamma v \\
u
\end{array}\right],
$$

onde a função $\chi$ satisfaz

$$
1+\chi^{\prime}(u)^{2}=\gamma^{2} \cosh ^{2} \chi(u), \quad \chi^{\prime \prime}(u)=\gamma^{2} \cosh \chi(u) \operatorname{senh} \chi(u) .
$$

Podemos assumir que $\chi^{\prime}(0)=0, \chi(u)>0$ e que $\chi$ está definida em um intervalo $\left(-u_{0}, u_{0}\right)$ com

$$
u_{0}=\int_{\chi(0)}^{\infty} \frac{d \chi}{\sqrt{\gamma^{2} \cosh ^{2} \chi-1}}=\int_{1}^{\infty} \frac{d x}{\sqrt{\left(x^{2}-\gamma^{2}\right)\left(x^{2}-1\right)}} .
$$

Daí temos

$$
u_{0}>\int_{1}^{\infty} \frac{d x}{x \sqrt{x^{2}-1}}=\frac{\pi}{2} .
$$

Desta forma temos que a altura de $\mathcal{G}_{\gamma}$ é maior que $\pi$, e esta tende a $\pi$ quando $\gamma \rightarrow 0$ e tende a $+\infty$ quando $\gamma \rightarrow 1$. Note que as curvas horizontais são equidistantes a geodésica de $\mathbb{H}^{2}$ dada por 
$\eta(v)=(\cosh \gamma v, 0, \operatorname{senh} \gamma v)$. De fato, como as curvas horizontais são da forma

$$
\xi(v)=(\cosh \chi(u) \cosh \gamma v, \operatorname{senh} \chi(u), \cosh \chi(u) \operatorname{senh} \gamma v)
$$

e a distância $d$ em $\mathbb{H}^{2}$ entre dois pontos $A, B \in \mathbb{H}^{2}$ é dada por (veja [CFK ${ }^{+97])}$

$$
\langle A, B\rangle_{\varepsilon}=-\cosh (d(A, B)),
$$

temos

$$
\begin{aligned}
& \langle\eta(v), \xi(v)\rangle_{\varepsilon}=-\cosh (d(\eta(v), \xi(v))) \\
\Rightarrow & -\cosh \chi(u) \cosh ^{2} \gamma v+\cosh \chi(u) \operatorname{senh}^{2} \gamma v=-\cosh (d(\eta(v), \xi(v))) \\
\Rightarrow & -\cosh \chi(u)=-\cosh (d(\eta(v), \xi(v))) \\
\Rightarrow & d(\eta(v), \xi(v))=\chi(u)
\end{aligned}
$$

para todo $v$.

Agora note que

$$
\frac{\partial x}{\partial u}=\left(\chi^{\prime}(u) \operatorname{senh} \chi(u) \cosh \gamma v, \chi^{\prime}(u) \cosh \chi(u), \chi^{\prime}(u) \operatorname{senh} \chi(u) \operatorname{senh} \gamma v, 1\right)
$$

$\mathrm{e}$

$$
\frac{\partial x}{\partial v}=(\cosh \chi(u) \gamma \operatorname{senh} \gamma v, 0, \cosh \chi(u) \gamma \cosh \gamma v, 0) .
$$

Temos também que

$$
\left\langle\frac{\partial x}{\partial u}, \frac{\partial x}{\partial u}\right\rangle_{-1}=1+\chi^{\prime}(u)^{2}, \quad\left\langle\frac{\partial x}{\partial u}, \frac{\partial x}{\partial v}\right\rangle_{-1}=0, \quad\left\langle\frac{\partial x}{\partial v}, \frac{\partial x}{\partial v}\right\rangle_{-1}=\gamma^{2} \cosh ^{2} \chi(u),
$$

logo a imersão $x$ é conforme.

O normal $\bar{N}$ à $\mathbb{H}^{2} \times \mathbb{R}$ em $\mathbb{L}^{4}$ é

$$
\bar{N}(u, v)=\left[\begin{array}{c}
\cosh \chi(u) \cosh \gamma v \\
\operatorname{senh} \chi(u) \\
\cosh \chi(u) \operatorname{senh} \gamma v \\
0
\end{array}\right] .
$$

Além disso, pelo que vimos na seção 3.2 , o normal $N$ à $\mathcal{G}_{\gamma}$ em $\mathbb{H}^{2} \times \mathbb{R}$ é

$$
N(u, v)=-\frac{1}{\gamma \cosh \chi(u)}\left[\begin{array}{c}
\operatorname{senh} \chi(u) \cosh \gamma v \\
\cosh \chi(u) \\
\operatorname{senh} \chi(u) \operatorname{senh} \gamma v \\
-\chi^{\prime}(u)
\end{array}\right] .
$$

Vamos calcular o operador forma $A_{\mathcal{G}_{\gamma}}$ de $\mathcal{G}_{\gamma}$. Temos:

$$
\begin{gathered}
\frac{\partial^{2} x}{\partial u^{2}}=\left[\begin{array}{c}
\chi^{\prime \prime}(u) \operatorname{senh} \chi(u) \cosh \gamma v+\chi^{\prime}(u)^{2} \cosh \chi(u) \cosh \gamma v \\
\chi^{\prime \prime}(u) \cosh \chi(u)+\chi^{\prime}(u)^{2} \operatorname{senh} \chi(u) \\
\chi^{\prime \prime}(u) \operatorname{senh} \chi(u) \operatorname{senh} \gamma v+\chi^{\prime}(u)^{2} \cosh \chi(u) \operatorname{senh} \gamma v \\
0
\end{array}\right] \\
\frac{\partial^{2} x}{\partial v^{2}}=\left[\begin{array}{c}
\cosh \chi(u) \gamma^{2} \cosh \gamma v \\
0 \\
\cosh \chi(u) \gamma^{2} \operatorname{senh} \gamma v \\
0
\end{array}\right],
\end{gathered}
$$




$$
\frac{\partial^{2} x}{\partial u \partial v}=\left[\begin{array}{c}
\chi^{\prime}(u) \operatorname{senh} \chi(u) \gamma \operatorname{senh} \gamma v \\
0 \\
\chi^{\prime}(u) \operatorname{senh} \chi(u) \gamma \cosh \gamma v \\
0
\end{array}\right]
$$

Portanto,

$$
\left\langle\frac{\partial^{2} x}{\partial u^{2}}, N\right\rangle_{-1}=-\gamma \operatorname{senh} \chi(u),\left\langle\frac{\partial^{2} x}{\partial v^{2}}, N\right\rangle_{-1}=\gamma \operatorname{senh} \chi(u),\left\langle\frac{\partial^{2} x}{\partial u \partial v}, N\right\rangle_{-1}=0 .
$$

Daí, $A_{\mathcal{G}_{\gamma}}$ é dado na base $\left\{\frac{\partial}{\partial u}, \frac{\partial}{\partial v}\right\}$ por

$$
\begin{aligned}
A_{\mathcal{G}_{\gamma}} & =\left[\begin{array}{cc}
\frac{1}{1+\chi^{\prime}(u)^{2}} & 0 \\
0 & \frac{1}{1+\chi^{\prime}(u)^{2}}
\end{array}\right]\left[\begin{array}{cc}
-\gamma \operatorname{senh} \chi(u) & 0 \\
0 & \gamma \operatorname{senh} \psi(u)
\end{array}\right] \\
& =-\frac{\gamma \operatorname{senh} \chi(u)}{1+\chi^{\prime}(u)^{2}}\left[\begin{array}{cc}
1 & 0 \\
0 & -1
\end{array}\right] .
\end{aligned}
$$

Além disso,

$$
\nu_{\mathcal{G}_{\gamma}}=\left\langle\frac{\partial}{\partial t_{\varepsilon}}, N\right\rangle=\frac{\chi^{\prime}(u)}{\gamma \cosh \psi(u)}
$$

$\mathrm{e}$

$$
\begin{aligned}
d x\left(T_{\mathcal{G}_{\gamma}}\right) & =\frac{\partial}{\partial t}-\nu_{\mathcal{G}_{\gamma}} N \\
& =(0,0,0,1)+\frac{\chi^{\prime}(u)}{\gamma^{2} \cosh \chi(u)^{2}}\left(\operatorname{senh} \chi(u) \cosh \gamma v, \cosh \chi(u), \operatorname{senh} \chi(u) \operatorname{senh} \gamma v, \chi^{\prime}(u)\right) \\
& =\frac{1}{1+\chi^{\prime}(u)^{2}} \frac{\partial x}{\partial v},
\end{aligned}
$$

$\log 0$

$$
T_{\mathcal{G}_{\gamma}}=\frac{1}{1+\chi^{\prime}(u)^{2}} \frac{\partial}{\partial v} .
$$

Proposição 3.4.4. A superfície conjugada do catenoide generalizado $\mathcal{G}_{\gamma}$ é o helicoide $\mathcal{H}_{\beta}$ tal que $\gamma^{2}+\beta^{2}=1$ e $\gamma, \beta$ com mesmo sinal.

Demonstração. Sejam $y_{1}(u)=\gamma \operatorname{senh} \chi(u)$ e $y_{2}(u)=\beta \cosh \phi(u)$. Note que

$$
\begin{aligned}
\left(y_{1}(u)^{2}+\gamma^{2}\right)\left(y_{1}(u)^{2}-\beta^{2}\right) & =\left(\gamma^{2} \operatorname{senh}^{2} \chi(u)+\gamma^{2}\right)\left(\gamma^{2} \operatorname{senh}^{2} \chi(u)-1+\gamma^{2}\right) \\
& =\left(\gamma^{2} \cosh ^{2} \chi(u)\right)\left(\gamma^{2} \cosh ^{2} \chi(u)-1\right) \\
& =\left(\gamma^{2} \cosh ^{2} \chi(u)\right)\left(\chi^{\prime}(u)^{2}\right) \\
& =y_{1}^{\prime}(u)^{2} .
\end{aligned}
$$

Da mesma forma temos

$$
\left(y_{2}(u)^{2}+\gamma^{2}\right)\left(y_{2}(u)^{2}-\beta^{2}\right)=y_{2}^{\prime}(u)^{2} .
$$

Portanto $y_{1}$ e $y_{2}$ são soluções da equação

$$
\left(y^{\prime}\right)^{2}=\left(y^{2}+\gamma^{2}\right)\left(y^{2}-\beta^{2}\right) .
$$

Derivando (3.32), obtemos

$$
y^{\prime \prime}=y\left(2 y^{2}+\gamma^{2}-\beta^{2}\right)
$$


logo $y_{1}$ e $y_{2}$ também são soluções de (3.33). Como

$$
1+\chi^{\prime}(u)^{2}=\gamma^{2} \cosh ^{2} \chi(u)=\gamma^{2}+\gamma^{2} \operatorname{senh}^{2} \chi(u)=\gamma^{2}+y_{1}(u)^{2},
$$

usando o fato de que $\chi^{\prime}(0)=0$ e $\gamma^{2}+\beta^{2}=1$, temos

$$
y_{1}(0)^{2}=\beta^{2} \text {. }
$$

Substituindo em (3.32) temos

$$
y_{1}^{\prime}(0)=0
$$

Além disso, como $\phi(0)=0$, temos

$$
y_{2}(0)=\beta \cosh \phi(0)=\beta \text {. }
$$

Substituindo em (3.32) temos também

$$
y_{2}^{\prime}(0)=0 .
$$

Sabemos que $\operatorname{senh} \chi(0)>0$, pois $\chi(0)>0, \operatorname{logo} y_{1}(0)$ tem o mesmo sinal de $\gamma$, e como $\gamma$ e $\beta$ têm o mesmo sinal, por (3.34) e (3.35), segue que $y_{1}(0)=y_{2}(0)$. Pelo teorema de existência e unicidade de soluções de EDO concluímos que $y_{1}=y_{2}$.

Precisamos mostrar que $\mathcal{G}_{\gamma}$ e $\mathcal{H}_{\beta}$ são localmente isométricos, $A_{\mathcal{H}_{\beta}}=J A_{\mathcal{G}_{\gamma}}, T_{\mathcal{H}_{\beta}}=J T_{\mathcal{G}_{\gamma}}$ e $\nu_{\mathcal{H}_{\beta}}=\nu_{\mathcal{G}_{\Gamma}}$.

1. Como

$$
\begin{aligned}
\phi^{\prime}(u) & =1+\beta^{2} \operatorname{senh}^{2} \phi(u)=1+\beta^{2} \cosh ^{2} \phi(u)-\beta^{2}=\gamma^{2}+y_{2}(u)^{2}=\gamma^{2}+y_{1}(u)^{2} \\
& =\gamma^{2}+\gamma^{2} \operatorname{senh}^{2} \chi(u)=\gamma^{2} \cosh ^{2} \chi(u)=1+\chi^{\prime}(u)^{2},
\end{aligned}
$$

segue que $\mathcal{G}_{\gamma}$ e $\mathcal{H}_{\beta}$ são localmente isométricos;

2. Utilizando o item anterior, $\operatorname{como} \beta \cosh \phi(u)=y_{1}(u)=y_{2}(u)=\gamma \operatorname{senh} \chi(u)$,

$$
\begin{aligned}
A_{\mathcal{H}_{\beta}} & =-\frac{\beta \cosh \phi(u)}{\phi^{\prime}(u)^{2}}\left[\begin{array}{ll}
0 & 1 \\
1 & 0
\end{array}\right]=-\frac{\gamma \operatorname{senh} \chi(u)}{1+\chi^{\prime}(U)^{2}}\left[\begin{array}{ll}
0 & 1 \\
1 & 0
\end{array}\right]=-\frac{\gamma \operatorname{senh} \chi(u)}{1+\chi^{\prime}(U)^{2}}\left[\begin{array}{cc}
0 & -1 \\
1 & 0
\end{array}\right]\left[\begin{array}{cc}
1 & 0 \\
0 & -1
\end{array}\right] \\
& =J A_{\mathcal{U}_{\alpha}} ;
\end{aligned}
$$

3.

$$
T_{\mathcal{H}_{\beta}}=\frac{1}{\phi^{\prime}(u)^{2}} \frac{\partial}{\partial v}=\frac{1}{1+\chi^{\prime}(u)^{2}} J \frac{\partial}{\partial u}=J T_{\mathcal{G}_{\gamma}}
$$

4. Temos

$$
\nu_{\mathcal{G}_{\gamma}}=\frac{\chi^{\prime}(u)}{\gamma \cosh \chi(u)}=\frac{y_{1}^{\prime}(u)}{\gamma^{2} \cosh ^{2} \chi(u)}=\frac{y_{1}^{\prime}(u)}{\gamma^{2}-\gamma^{2} \operatorname{senh}^{2} \chi(u)}=\frac{y_{1}^{\prime}(u)}{\gamma^{2}-y_{1}(u)^{2}}
$$

e

$$
\begin{aligned}
\nu_{\mathcal{H}_{\beta}} & =\frac{\beta \operatorname{senh} \phi(u)}{\phi^{\prime}(u)}=\frac{\beta \phi^{\prime}(u) \operatorname{senh} \phi(u)}{\phi^{\prime}(u)^{2}}=\frac{y_{2}^{\prime}(u)}{1+\beta^{2} \operatorname{senh}^{2} \phi(u)}=\frac{y_{2}^{\prime}(u)}{1+\beta^{2} \cosh ^{2} \phi(u)-\beta^{2}} \\
& =-\frac{y_{2}^{\prime}(u)}{\gamma^{2}-y_{2}(u)^{2}},
\end{aligned}
$$

$\operatorname{logo} \nu_{\mathcal{H}_{\beta}}=\nu_{\mathcal{G}_{\gamma}}$.

Portanto segue que $\mathcal{H}_{\beta}$ é superfície conjugada de $\mathcal{G}_{\gamma}$, como queríamos. 


\subsection{Mais exemplos no caso hiperbólico}

Daremos agora exemplos de Superfícies Riemannianas admitindo duas imersões isométricas em $\mathbb{H}^{2} \times \mathbb{R}$ que não são associadas, o que não ocorre em $\mathbb{R}^{3}$. Em [Ear08], Sá Earp classificou as superfícies mínimas em $\mathbb{H}^{2} \times \mathbb{R}$ que são invariantes por um grupo a um parâmetro de hyperbolic screw motions, ou seja, cada elemento desse grupo é composição de uma translação hiperbólica com uma translação vertical. Estas superfícies são descritas por uma família a 2 parâmetros $\left(S_{l, d}\right)_{(l, d) \in \mathbb{R}^{2}}$. Sá Earp observou que duas superfícies $S_{l, d}$ e $S_{\underline{l}, \underline{d}}$ são isométricas mas não associadas se $d^{2}>1, \underline{d}^{2}<1$, e

$$
\frac{\left(d^{2}-1\right)}{\left(l^{2}+1\right)}=\frac{\left(1-\underline{d}^{2}\right)}{\left(\underline{d}^{2}+\underline{l}^{2}\right)}
$$

Sejam $d, l \in \mathbb{R}$, com $d^{2}>1$. Vamos construir um modelo para tais superfícies. Tomemos $\mathbb{R}^{2}$ munido da métrica

$$
d s^{2}=d u^{2}+\Lambda(u)^{2} d v^{2}
$$

onde

$$
\Lambda(u)=\sqrt{\left(d^{2}-1\right) \cosh ^{2} u+l^{2}+1} .
$$

Vamos considerar o referencial ortonormal $\left(e_{1}, e_{2}\right)$ definido por

$$
e_{1}=\frac{\partial}{\partial u}, \quad e_{2}=\frac{1}{\Lambda(u)} \frac{\partial}{\partial v} .
$$

Sua base dual é dada por

$$
\omega^{1}=d u, \quad \omega^{2}=\Lambda(u) d v .
$$

Pela equação (1.4), temos que

$$
\begin{aligned}
& -\omega_{1}^{2} \wedge \omega^{1}=d \omega^{2}=\Lambda^{\prime}(u) d u \wedge d v=-\frac{\Lambda^{\prime}(u)}{\Lambda(u)} \omega^{2} \wedge \omega^{1} \\
& \Rightarrow \quad \omega_{1}^{2}=\frac{\Lambda^{\prime}(u)}{\Lambda(u)} \omega^{2}=\Lambda^{\prime}(u) d v
\end{aligned}
$$

daí

$$
d \omega_{1}^{2}=\Lambda^{\prime \prime}(u) d u \wedge d v=\frac{\Lambda^{\prime \prime}(u)}{\Lambda(u)} \omega^{1} \wedge \omega^{2} .
$$

Utilizando (3.14) segue que

$$
K=-\frac{\Lambda^{\prime \prime}(u)}{\Lambda(u)}
$$

Mais explicitamente, temos

$$
K=-1+\frac{\left(l^{2}+1\right)\left(d^{2}+l^{2}\right)}{\Lambda(u)^{4}} .
$$

Além disso podemos verificar que as funções $\nu$ e $\underline{\nu}$ dadas por

$$
\begin{gathered}
\nu(u)=\frac{\left(d^{2}-1\right) \cosh ^{2} u}{\Lambda(u)^{2}}=1-\frac{l^{2}+1}{\Lambda(u)^{2}} \\
\underline{\nu}(u)=\frac{\left(d^{2}-1\right) \operatorname{senh}^{2} u}{\Lambda(u)^{2}}=1-\frac{d^{2}+l^{2}}{\Lambda(u)^{2}}
\end{gathered}
$$

satisfazem (3.7) e (3.8). Pelo teorema 3.1.7, existem imersões isométricas mínimas $f$ e $g$ de $\left(\mathbb{R}^{2}, d s^{2}\right)$ em $\mathbb{H}^{2} \times \mathbb{R}$ com funções ângulo $\nu$ e $\underline{\nu}$, respectivamente. Como $d^{2}>1$, nota-se facilmente que $\nu \neq \underline{\nu}$, $\operatorname{logo} f$ e $g$ não pertencem a mesma família associada.

As funções $\nu$ e $\underline{\nu}$ são as funções ângulo de $S_{l, d}$ e $S_{\underline{l}, \underline{d}}$, respectivamente, com $\underline{d}^{2}<1$ e satisfazendo 
(3.37). Como pode ser visto em [Ear08], a métrica em $S_{\underline{l}, \underline{d}}$ é dada por

$$
d s^{2}=d u^{2}+\underline{\Lambda}(u)^{2} d v^{2},
$$

com

$$
\underline{\Lambda}(u)=\sqrt{\left(1-\underline{d}^{2}\right) \cosh ^{2} u+\underline{d}^{2}+\underline{l}^{2}}
$$

Note que

$$
\begin{aligned}
\Lambda(u)^{2} & =\left(d^{2}-1\right) \cosh ^{2} u+l^{2}+1=\left(l^{2}+1\right) \frac{\left(1-\underline{d}^{2}\right)}{\underline{d}^{2}+\underline{l}^{2}} \cosh ^{2} u+l^{2}+1 \\
& =\frac{\left(l^{2}+1\right)}{\underline{d}^{2}+\underline{l}^{2}}\left[\left(1-\underline{d}^{2}\right) \cosh ^{2} u+\underline{d}^{2}+\underline{l}^{2}\right]=\frac{\left(l^{2}+1\right)}{\underline{d}^{2}+\underline{l}^{2}} \underline{\Lambda}(u)^{2}
\end{aligned}
$$

$\mathrm{e}$

$$
\begin{aligned}
\underline{\nu}(u) & =1-\frac{d^{2}+l^{2}}{\Lambda(u)^{2}}=1-\frac{\left(l^{2}+1\right)\left(\underline{l}^{2}+1\right)}{\Lambda(u)^{2}\left(\underline{d}^{2}+\underline{l}^{2}\right)} \\
& =1-\frac{\left(\underline{l}^{2}+1\right)}{\underline{\Lambda}(u)^{2}}
\end{aligned}
$$

logo podemos concluir que, a menos da mudança de coordenadas $v \mapsto \frac{\left(l^{2}+1\right)}{\underline{d}^{2}+\underline{l}^{2}} v$, as superfícies $S_{l, d}$ e $S_{\underline{l}, \underline{d}}$ não pertencem a mesma família associada.

Note agora que as superfícies $S_{l, d}$ e $S_{0, \delta}, \operatorname{com} \delta=\sqrt{1+\frac{\left(d^{2}-1\right)}{\left(l^{2}+1\right)}}$ são associadas. De fato, como $d^{2}>1$, temos $\delta^{2}>1$, e como

$$
\begin{aligned}
\Lambda(u)^{2} & =\left(d^{2}-1\right) \cosh ^{2} u+l^{2}+1=\left(l^{2}+1\right)\left[\frac{\left(d^{2}-1\right)}{l^{2}+1} \cosh ^{2} u+1\right] \\
& =\left(l^{2}+1\right)\left[\left(\delta^{2}-1\right) \cosh ^{2} u+1\right]
\end{aligned}
$$

temos que

$$
\nu(u)=1-\frac{l^{2}+1}{\Lambda(u)^{2}}=1-\frac{1}{\left(\delta^{2}-1\right) \cosh ^{2} u+1},
$$

logo fazendo a mudança de coordenadas $v \mapsto \sqrt{l^{2}+1} v$, temos que as superfícies são isométricas e possuem a mesma função ângulo, logo pertencem a mesma família associada. 


\section{Referências Bibliográficas}

[Bon67] Ossian Bonnet. Mémoire sur la théorie des surfaces applicables sur une surface donnée. Deuxième partie. Gauthier-Villars, 1867. 1

[CC10] Qun Chen e Qing Cui. Isometric immersions into $\mathbb{S}^{m} \times \mathbb{R}$ and $\mathbb{H}^{m} \times \mathbb{R}$ with high codimensions. Results Math., 57(3-4):319-333, 2010. 1

$\left[\mathrm{CFK}^{+}\right.$97] James W Cannon, William J Floyd, Richard Kenyon, Walter R Parry et al. Hyperbolic geometry. Flavors of geometry, 31:59-115, 1997. 60, 70

[Daj90] Marcos Dajczer. Submanifolds and isometric immersions, volume 13. Publish or Perish, 1990. 3

[Dan07] Benoît Daniel. Isometric immersions into 3-dimensional homogeneous manifolds. Commentarii Mathematici Helvetici, 82(1):87-131, 2007.

[Dan09] Benoît Daniel. Isometric immersions into $\mathbb{S}^{n} \times \mathbb{R}$ and $\mathbb{S}^{n} \times \mathbb{R}$ and applications to minimal surfaces. Transactions of the American Mathematical Society, 361(12):6255-6282, 2009. 1

[Dan15] Benoît Daniel. Minimal isometric immersions into $\mathbb{S}^{2} \times \mathbb{R}$ and $\mathbb{H}^{2} \times \mathbb{R}$. Indiana Univ. Math. J., 64(5):1425-1445, 2015. 1

[dC76] Manfredo Perdigão do Carmo. O método do referencial móvel. Instituto de Matemática Pura e Aplicada, 1976. 3

[dC79] Manfredo Perdigão do Carmo. Geometria riemanniana, volume 10 of Projeto Euclides. Instituto de Matemática Pura e Aplicada, Rio de Janeiro, 1979. 3, 4, 45, 46, 50

[Ear08] Ricardo Sa Earp. Parabolic and hyperbolic screw motion surfaces in $\mathbb{H}^{2} \times \mathbb{R}$. Journal of the Australian Mathematical Society, 85(1):113-143, 2008. 73, 74

[HSET08] Laurent Hauswirth, Ricardo Sa Earp e Eric Toubiana. Associate and conjugate minimal immersions in $M \times \mathbf{R}$. Tohoku Math. J. (2), 60(2):267-286, 2008. 1

[Lee12] John Lee. Introduction to Smooth Manifolds, volume 218. Springer Science \& Business Media, 2012. 5, 33

[NR02] Barbara Nelli e Harold Rosenberg. Minimal surfaces in $\mathbb{H}^{2} \times \mathbb{R}$. Bulletin of the Brazilian Mathematical Society, 33(2):263-292, 2002. 61

[Pet06] Peter Petersen. Riemannian geometry, volume 171. Springer, 2006. 44

[Rey93] William F Reynolds. Hyperbolic geometry on a hyperboloid. The American mathematical monthly, 100(5):442-455, 1993.

[Ros02] Harold Rosenberg. Minimal surfaces in $\mathbb{M}^{2} \times \mathbb{R}$. Illinois J. Math., 46(4):1177-1195, 2002. 
[Spi79] Michael Spivak. A comprehensive introduction to differential geometry. Vol. IV. Publish or Perish, Inc., Wilmington, Del., 1979. 1

[Ten71] Keti Tenenblat. On isometric immersions of Riemannian manifolds. Bol. Soc. Brasil. Mat., 2(2):23-36, 1971. 1, 17

[Xin03] Yuanlong Xin. Minimal submanifolds and related topics, volume 8. World Scientific, 2003. 44,45 